\title{
PineAPPL: combining EW and QCD corrections for fast evaluation of LHC processes
}

\section{S. Carrazza, ${ }^{a}$ E.R. Nocera, ${ }^{b}$ C. Schwan ${ }^{a}$ and M. Zaro ${ }^{a}$}

${ }^{a}$ Tif Lab, Dipartimento di Fisica, Università di Milano and INFN, Sezione di Milano, via Celoria 16, 20133 Milano, Italy

${ }^{b}$ Nikhef Theory Group,

Science Park 105, 1098 XG Amsterdam, The Netherlands

E-mail: stefano.carrazza@mi.infn.it, e.nocera@nikhef.nl,

christopher.schwan@mi.infn.it, marco.zaro@mi.infn.it

ABSTRACT: We introduce PINEAPPL, a library that produces fast-interpolation grids of physical cross sections, computed with a general-purpose Monte Carlo generator, accurate to fixed order in the strong, electroweak, and combined strong-electroweak couplings. We demonstrate this unique ability, that distinguishes PINEAPPL from similar software available in the literature, by interfacing it to MADGRAPH5_AMC@NLO. We compute fast-interpolation grids, accurate to next-to-leading order in the strong and electroweak couplings, for a representative set of LHC processes for which EW corrections may have a sizeable effect on the accuracy of the corresponding theoretical predictions. We formulate a recommendation on the format of the experimental deliverables in order to consistently compare them with computations that incorporate EW corrections, and specifically to determine parton distribution functions to the same accuracy.

KeYwords: NLO Computations, QCD Phenomenology

ArXiv EPrint: 2008.12789 


\section{Contents}

$\begin{array}{llr}1 & \text { Introduction } & 1\end{array}$

2 PDF-independent storage of phase-space weights with PineAPPL 4

2.1 The PineAPPL library 4

2.2 Cross sections in a multi-coupling expansion 5

$\begin{array}{lll}2.2 .1 & \text { Grid representations } & 7\end{array}$

$\begin{array}{lll}2.3 & \text { Perturbative orders } & 10\end{array}$

2.3.1 Example: Drell-Yan lepton-pair production at the LHC 10

3 Validation and interpretation of PineAPPL grids $\quad 11$

$\begin{array}{ll}3.1 \text { Computational settings } & 11\end{array}$

$\begin{array}{lll}3.2 & \text { Results for specific processes and measurements } & 13\end{array}$

$\begin{array}{lll}3.2 .1 & \text { Drell-Yan lepton pair production } & 14\end{array}$

$\begin{array}{lll}3.2 .2 & \text { Top-quark pair production } & 18\end{array}$

3.2.3 Z-boson production with non-zero transverse momentum 20

4 Subtraction of EW effects from data $\quad 22$

5 Conclusions and outlook $\quad 24$

A Installation and usage of PineAPPL 25

A.1 Demonstration of pineappl 26

$\begin{array}{lll}\text { A.2 Sample runcard for mg5_aMC@NLO } & 29\end{array}$

A.3 Example Monte Carlo program in $\mathrm{C}++\quad 29$

A.4 Installation 33

$\begin{array}{ll}\text { B Parton luminosities } & 34\end{array}$

$\begin{array}{ll}\text { C Low statistics and complementary results } & 40\end{array}$

\section{Introduction}

With the recent completion of Run II, the Large Hadron Collider (LHC) has accumulated data from an integrated luminosity of approximately $150 \mathrm{fb}^{-1}[1]$. This represents only a small fraction of the anticipated $3000 \mathrm{fb}^{-1}$ that will eventually be recorded in the forthcoming twenty years of LHC operation. Nevertheless the statistical uncertainty of the data has already shrunk to unprecedentedly small values, typically $1 \%$ or less, a fact that will allow for precision tests of the Standard Model (SM) and for indirect searches of New Physics 
only if theoretical predictions become comparatively precise. This entails the computation of additional higher-order contributions to the fixed-order perturbative expansion, on the one hand, and an increasingly sophisticated determination of the Parton Distribution Functions (PDFs) of the proton [2,3], on the other hand.

In the first respect, because Quantum Chromodynamics (QCD) dominates the interactions occurring within colliding protons at the LHC, much effort has been devoted to the computation of higher-order QCD corrections: fully-differential next-to-leading order (NLO) results, possibly matched to a parton shower, are currently automated in various general-purpose Monte Carlo generators [4-6] (see also ref. [7] for a review), while an increasing number of next-to-next-to-leading order (NNLO) predictions are becoming available for processes with various degrees of inclusiveness (see e.g. ref. [8] and references therein). The computation of higher-order corrections in the electroweak (EW) and combined QCD+EW theory has also witnessed a comprehensive progress. Frameworks were developed in which the QCD and EW couplings are simultaneously treated as small parameters in the perturbative expansion, and the computation of theoretical predictions, accurate to NLO in both (including multi-coupling QCD-EW terms), is automated [9-11]. For an extensive and recent review, see ref. [12].

In the second respect, contemporary PDF sets [13-15] incorporate a significant amount of LHC data, which is analysed with NNLO QCD theory by default. No EW corrections are systematically included in the theoretical description of the experimental observables to which PDFs are optimised, except for QED effects if a photon PDF is determined [16-22]. The QED contribution is included in the matrix element (at the lowest order for the LHC processes considered) and in the DGLAP splitting functions at leading order [23]. Inclusion of NLO QCD-QED combined corrections in the evolution is in principle also possible [24], and should be required to analyse the data within consistent perturbative accuracy if higher-order EW and QCD corrections are also included in partonic cross sections. The resulting relative PDF uncertainty - which accounts only for the uncertainty of the data and of residual methodological inefficiencies inherent to each PDF determination - can be as low as $1 \%$ at the EW scale [14]. Theoretical uncertainties, possibly of comparable size (e.g. from missing higher-order terms in the QCD perturbative expansion), have started to be represented into PDF uncertainties only very recently $[25,26]$.

The two respects are intertwined, as they both concur to determine the accuracy of the theoretical predictions that are matched to the precision of the data. In particular, taking advantage of the automation pioneered in refs. [9-11], perturbative corrections that arise from the simultaneous expansion in both the QCD and EW couplings should start to be incorporated in calculations for LHC processes as standard. The reason is twofold. First, one expects NNLO QCD and NLO EW corrections to be of comparable size because, at the EW scale, the QCD and EW running couplings become similar, $\alpha_{\mathrm{s}}^{2} \sim \alpha$. If NNLO QCD corrections are included by default in the computations, NLO EW corrections should be taken into account as well. Second, the virtual exchange of soft or collinear weak bosons leads to Sudakov logarithms [27, 28] (see also ref. [12] and references therein), which can make the coefficients of the EW series grow faster than their QCD counterparts. This behaviour is relevant in phase-space regions associated with large mass scales (roughly 
of the order of a TeV), where several LHC data sets (e.g. Z-boson transverse-momentum distributions) enter both the validation of the SM and the search for new physics.

The consistent inclusion of QCD and EW corrections in precision computations for LHC processes entails the solution of two separate problems. First, a problem of efficiency: fast-interpolation grids should be constructed, whereby partonic matrix elements, accurate to NLO QCD+EW, are precomputed in such a way that the numerical convolution with generic input PDFs can be efficiently approximated by means of interpolation techniques. Such grids are essential whenever the evaluation of the hadronic cross section needs to be performed a large number of times, as is the case in the evaluation of scale variations or of PDF fits. While two formats already exist for these grids, APPLGRID [29] and FASTNLO [30-32], none of them supports the inclusion of EW corrections nor the interface to a Monte Carlo generator accurate to NLO QCD+EW. Second, a problem of consistency: the way in which EW effects may (or may not) be folded into the data varies across different experimental analyses, a fact that challenges their consistent theoretical interpretation. Examples are the subtraction of background processes which should not be considered as such (e.g. the $t$-channel photon-induced component in neutral-current Drell-Yan, which is not a separate process beyond leading order) or of just a part of the EW effects (e.g. multiple-photon radiation from light particles in the final state of neutralor charged-current Drell-Yan, especially with electrons). Be that as it may, if EW effects are systematically included in theoretical predictions, they should not be subtracted from experimental results, otherwise they will be double counted.

In this paper we address the first of these two problems. Specifically, we develop PINEAPPL, a library that allows any user to generate fast-interpolation grids, accurate to any fixed order in the QCD and EW couplings. The library supports variations of the factorisation and renormalisation scales, and can be extended to include resummation, and matching with a photon- and/or parton-shower. The grids in the new PINEAPPL format complement those (accurate to fixed order in the strong coupling only) that can be generated in the APPLGRID and FAstNLO formats. The PINEAPPL library is interfaced to MADGRAPH5_AMC@NLO (MG5_AMC henceforth), with which it has been developed and tested, however it can be easily used with any Monte Carlo generator, e.g. SHERPA [10]. In this respect, PINEAPPL extends to EW corrections the scope of AMCFAst [33] and MCGRId [34, 35].

The paper is organised as follows. In section 2 we introduce PINEAPPL, we describe its features, we illustrate its operation, and we assess its performance. In section 3 we validate PINEAPPL and demonstrate its capabilities by computing fast-interpolation grids, accurate to NLO QCD and NLO QCD+EW, for a representative set of LHC processes for which EW corrections may have a sizeable effect on the accuracy of the theoretical predictions. In section 4 we try to detail in a more comprehensive manner the doublecounting problem sketched above, the solution of which, however, remains beyond the scope of the current work. We provide our conclusions and an outlook in section 5. Examples of usage and the installation of PINEAPPL are provided in appendix A; appendix B collects the parton luminosities for each process considered in section 3 ; and appendix $\mathrm{C}$ complements some of the results presented in section 3 . 


\section{PDF-independent storage of phase-space weights with PineAPPL}

In this section we introduce PINEAPPL. We first describe the general scope and features of the library in comparison to APPLGRID and FASTNLO in section 2.1. Section 2.2 gives the details and, in particular, describes the problem of computing Monte Carlo weights for cross sections in a multi-coupling expansion; this section may be read first by readers unfamiliar with the programs mentioned previously. In section 2.3 we discuss a few properties of multi-coupling-expanded predictions.

\subsection{The PineAPPL library}

PINEAPPL is a new library which stores phase-space weights of a Monte Carlo (MC) integration of a fixed-order calculation independently from the chosen PDFs. The task of computing predictions for physical observables is therefore split into two steps: 1) the generation of the grids, i.e. the files in which the $\mathrm{MC}$ weights are stored, and 2) the convolution of these grids with a set of PDFs. The advantage of this method is that the time-consuming step 1) has to be performed only once, and that step 2) is reduced to a fast convolution of a given grid with one (or more) PDF set(s).

The convolution is typically done in few seconds or less, a fact that offers at least two applications:

1. the study of the PDF-dependence of the observables; e.g. PDF set comparisons, PDF uncertainty computations, $\alpha_{\mathrm{s}}$ variations, etc., and

2. the determination of PDF sets themselves; together with the corresponding experimental data, the grids constitute two important ingredients for a PDF fit.

These features are common to both PineAPPL and APPLGRID [29] or FAstNLO [30-32]. However, in comparison to the last two pieces of code, PINEAPPL allows one to include also higher-order corrections due to EW, and in general combined QCD-EW, effects for the first time. Documenting this extension, and how to interface PINEAPPL with a generalpurpose matrix-element generator, is the main goal of this paper.

In particular, PINEAPPL supports the following features.

- The inclusion of perturbative corrections (fixed-order, i.e. without parton-shower matching) with any given set of powers of $\alpha, \alpha_{\mathrm{s}}$, in particular including combined QCD-EW corrections.

- The support for non-coloured initial-state partons, such as photon-initiated contributions, and, more generally, arbitrary initial-state combinations, e.g. leptonic initial states [36, 37].

- The estimate of theory uncertainties via variations of the renormalisation and factorisation scale (the electroweak coupling is assumed to be scale-independent, consistently with the most common renormalisation schemes).

On a more technical level, we point out the following additional features of PINEAPPL. 
- PINEAPPL comes with the shell command pineappl, which performs convolutions on the command line, without requiring the user to write a new program. In addition to convolutions, the shell command can also print how the luminosity function is constructed, which perturbative orders are stored in the grids, their size, the size of each partonic channel, etc., separately for each bin. See appendix A.1 for details.

- PINEAPPL offers an easy-to-use interface written in the $\mathrm{C}$ programming language, that allows MC integrators to read and write PINEAPPL grids. $\mathrm{C}$ was chosen because it can be easily interfaced with both Fortran and $\mathrm{C}++$, the two main programming languages in which most MC integrators are written. The interface consists of roughly thirty functions, among which only a handful are needed in practice. We also provide and support a Python package based on this C interface. See appendix A.3 for an example.

- PineAPPL has been explicitly interfaced to MG5_AMC (v3+) $[5,11],{ }^{1}$ see appendix A.2 for an example of a runcard. The usage of MG5_AMC(v3+) + PINEAPPL is similar to that of MG5_AMC(v2) + AMCFAST + APPLGRID. While the latter toolchain proved to be sufficiently efficient for NLO QCD calculations of typical kinematic distributions (with a few bins), this is no longer so when taking into account NLO EW calculations and, with more precise data, distributions that are typically more complex and have more bins. Taking into account these two reasons, we find that the memory usage of APPLGRID is no longer satisfiable. In particular, using APPLGRID for NLO QCD+EW ${ }^{2}$ typically requires close to $120 \mathrm{~GB}$ for highmass Drell-Yan with 13 bins (see section 3.2.1). The memory usage is substantially reduced to roughly 1 GB after "optimisation" of the grids, i.e. an optimisation of the grid representation in memory. However, this requires a two-step procedure: first, to produce an unoptimised grid in order to identify those parts where the cross section is either zero or extremely suppressed; second, after those parts are removed, to fill an optimised grid with a small number of bins. PINEAPPL avoids this by using a more space-efficient representation from the start. This leads to substantially faster run times, in particular for simple processes, because the grids do not need to be optimised and their combination is faster.

\subsection{Cross sections in a multi-coupling expansion}

Fixed-order partonic cross sections $a+b \rightarrow X$ supported by PINEAPPL are written as an expansion in powers of the strong coupling $\alpha_{\mathrm{s}}$, the electromagnetic coupling $\alpha$, and the

\footnotetext{
${ }^{1} \mathrm{~A}$ version of MG5_AMC interfaced to PINEAPPL can be downloaded from https://launchpad.net/mg5amc-pineappl/trunk, and it will be included as standard starting from the 3.0.4 release of MG5_AMC.

${ }^{2}$ For this a modified version of APPLGRID was developed which added support for photon-initiated processes and EW corrections.
} 
logarithms of $\xi_{\mathrm{R}}=\mu_{\mathrm{R}} / Q$ and $\xi_{\mathrm{F}}=\mu_{\mathrm{F}} / Q$,

$$
\begin{aligned}
\frac{\mathrm{d} \sigma_{a b}}{\mathrm{~d} \mathcal{O}}\left(x_{1}, x_{2}, \mathcal{O}, \xi_{\mathrm{R}}, \xi_{\mathrm{F}}\right)= & \sum_{k, l, m, n} \alpha_{\mathrm{s}}^{k}\left(\xi_{\mathrm{R}}^{2} Q^{2}\right) \alpha^{l} \log ^{m}\left(\xi_{\mathrm{R}}^{2}\right) \log ^{n}\left(\xi_{\mathrm{F}}^{2}\right) W_{a b}^{(k, l, m, n)} \\
& \times\left(x_{1}, x_{2}, Q^{2}, \mathcal{O}\right) .
\end{aligned}
$$

This cross section is differential with respect to the observable $\mathcal{O}$, which, in general, is a function of phase space and subject to the usual conditions (soft and collinear safety, etc.).

In experiments, but also for many calculations where the phase-space integration is performed using MC techniques, finite statistics does not allow for the exact reconstruction of the dependence of the cross section on the observable $\mathcal{O}$. Instead, it is sufficient to approximate the derivative using a piecewise-constant function,

$$
W_{a b}^{(k, l, m, n)}\left(x_{1}, x_{2}, Q^{2}, \mathcal{O}\right) \approx \sum_{o=1}^{M} \frac{\Theta\left(\mathcal{O}_{o}^{\min } \leq \mathcal{O}<\mathcal{O}_{o}^{\max }\right)}{\mathcal{O}_{o}^{\max }-\mathcal{O}_{o}^{\min }} w_{a b}^{(k, l, m, n, o)}\left(x_{1}, x_{2}, Q^{2}\right)
$$

which uses $M$ bins with limits $\left\{\mathcal{O}_{o}^{\min }, \mathcal{O}_{o}^{\max }\right\}_{o=1}^{M}$ to partition a finite range of the observable,

$$
\mathcal{O}_{0}^{\min }<\mathcal{O}_{0}^{\max }=\mathcal{O}_{1}^{\min }<\ldots<\mathcal{O}_{M-1}^{\max }=\mathcal{O}_{M}^{\min }<\mathcal{O}_{M}^{\max } .
$$

The Ellis-Sexton scale $Q^{2}$, if chosen dynamically, depends on the phase space, however we assume the fractions $\xi_{\mathrm{R}}$ and $\xi_{\mathrm{F}}$ to be phase-space constants in any case. This allows for variations around the central scale choice $\xi_{\mathrm{R}}=\xi_{\mathrm{F}}=1$, but it does not otherwise allow for arbitrary changes of the scale. The terms with powers $m>0$ and $n>0$ vanish for the central scale choice and are only required for variations of the factorisation and renormalisation scales. To estimate the perturbative QCD uncertainty - no EW uncertainty is covered by this method - one typically uses scale variations. Common prescriptions are 7-point and 9-points scale variations, which evaluate the cross section using respectively the following values

$$
\begin{aligned}
& \left(\xi_{\mathrm{R}}, \xi_{\mathrm{F}}\right)_{7-\mathrm{pt}} \in\left\{(1,1),\left(\frac{1}{2}, \frac{1}{2}\right),(2,2),\left(\frac{1}{2}, 1\right),\left(1, \frac{1}{2}\right),(2,1),(1,2)\right\} \\
& \left(\xi_{\mathrm{R}}, \xi_{\mathrm{F}}\right)_{9-\mathrm{pt}} \in\left\{(1,1),\left(\frac{1}{2}, \frac{1}{2}\right),(2,2),\left(\frac{1}{2}, 1\right),\left(1, \frac{1}{2}\right),(2,1),(1,2),\left(\frac{1}{2}, 2\right),\left(2, \frac{1}{2}\right)\right\} .
\end{aligned}
$$

The (asymmetric) uncertainties are then given as the minimum and maximum value (the envelope), measured from the central value $(1,1)$. As is clear from eq. (2.1), the EW coupling $\alpha$ is assumed not to be a dynamically varying coupling, but instead a constant over phase space. This, however, includes the most common choices of the coupling, which are (not necessarily in this order), $\alpha(0), \alpha\left(M_{\mathrm{Z}}\right)$, and $\alpha_{G_{\mu}}$.

The problem that PINEAPPL solves can now be described: approximately reconstruct the functions

$$
w_{a b}^{(k, l, m, n, o)}\left(x_{1}, x_{2}, Q^{2}\right)
$$

of eq. (2.2) from a set of $N$ function evaluations for specific momentum fractions, scales, and values of the observable

$$
\left\{x_{1}^{(i)}, x_{2}^{(i)}, Q_{i}^{2}, \mathcal{O}_{i}\right\}_{i=1}^{N},
$$


given by the MC integrator together with the corresponding value of the weights, eq. (2.6). This problem is solved by finding an appropriate representation of eq. (2.7) and is described in section 2.2.1. Using eq. (2.1) and

$$
\begin{aligned}
\frac{\mathrm{d} \sigma}{\mathrm{d} \mathcal{O}}\left(\mathcal{O}, \xi_{\mathrm{R}}, \xi_{\mathrm{F}}\right)= & \sum_{a, b} \int_{0}^{1} \mathrm{~d} x_{1} \int_{0}^{1} \mathrm{~d} x_{2} \int_{Q_{\min }^{2}}^{Q_{\max }^{2}} \mathrm{~d} Q^{2} f_{a}\left(x_{1}, \xi_{\mathrm{F}}^{2} Q^{2}\right) f_{b}\left(x_{2}, \xi_{\mathrm{F}}^{2} Q^{2}\right) \\
& \times \sigma_{a b}\left(x_{1}, x_{2}, Q^{2}, \xi_{\mathrm{R}}, \xi_{\mathrm{F}}\right),
\end{aligned}
$$

PINEAPPL can then quickly calculate hadronic cross sections for an arbitrary number of PDF sets and perform scale variations.

Note that we have omitted the dependence of the weights $w$, the observable $\mathcal{O}$, and the scales $\mu_{\mathrm{F}}, \mu_{\mathrm{R}}, Q^{2}$ on the specific kinematics for which they are computed. Indeed, beyond LO, different kinematic contributions have to be considered (in ref. [33], for example, they are labelled with an index $\alpha$, see eq. (12) therein). In the FKS subtraction scheme [38, 39] employed in MG5_AMC one type of kinematics for each counterterm (soft, collinear, and soft-collinear) is needed, however this is not the general case. In Catani-Seymour subtraction [40, 41], for example, different dipoles have different phase spaces and therefore different scales. PINEAPPL remains completely blind to this fact, and a consistent treatment is ensured by filling each event into a grid using the numerical value of $Q^{2}$.

\subsubsection{Grid representations}

We now explain the details of how the phase-space weights $w_{a b}$ in eq. (2.3) are represented.

4-tuples. A straightforward representation is given by 4-tuples, i.e. a list of the momentum fractions $x_{1}$ and $x_{2}$, the scale $Q^{2}$, and the phase-space weight $w$ for each phase-space point; 4-tuples are sufficient to reconstruct the differential cross sections. For each combination $(a, b, k, l, m, n, o)$ (see section 2.2 for their definition) we save the following 4-tuples,

$$
\left\{x_{1}^{i}, x_{2}^{i}, Q_{i}^{2}, w_{a b}^{(k, l, m, n, o)}\left(x_{1}^{i}, x_{2}^{i}, Q_{i}^{2}, \mathcal{O}_{i}\right)\right\}_{i=1}^{N} .
$$

The reconstruction of the differential cross sections are then done by simply multiplying the phase-space weights $w$ with PDFs evaluated with the correct arguments given in the 4 -tuple and summing over all indices $a, b, k, l, m, n, o$, and $i$.

The 4-tuple representation is very easy to implement and test. Furthermore, it reproduces the exact numerical value that is also calculated by the MC integrator. However, the price one has to pay is the size of the 4-tuples. For example, NLO QCD+EW Drell-Yan lepton-pair production (see section 2.3.1 and section 3.2.1) needs $159 \mathrm{MB}$ of storage for a target precision of $1 \%$ of the integrated cross section. While this is an acceptable size, increasing the precision by an order of magnitude would require roughly 100 times the size, due to the Monte Carlo convergence that goes as $1 / \sqrt{N}$ with $N$ being the number of 4tuples. With increasing size also the speed of the convolution degrades, because it basically becomes bound by the speed with which the 4 -tuples can be read from disk. However, due to the uncompressed nature of this representation it can serve as an intermediate format to develop and quickly cross check more space-efficient representations, one of which we will discuss next. 
Lagrange-interpolation grid. A different strategy is to partition a subset $H$ of the $\left(x_{1}, x_{2}, Q^{2}\right)$ space,

$$
H=\left[x_{\min }, x_{\max }\right]^{2} \times\left[Q_{\min }^{2}, Q_{\max }^{2}\right] \ni\left(x_{1}, x_{2}, Q^{2}\right)
$$

along each axis into a small number of bins and to insert the phase-space weights $w$ into the corresponding discrete bin. Using the bin centres and their values one already has a straightforward representation of eq. (2.6), but given a finite number of bins this approach usually yields an insufficient approximation for the cross section. Increasing the number of bins improves the precision, but it also increases the space requirements. This problem in turn is solved using interpolation methods, which increase the precision using the same number of bins.

We use the "Lagrange-interpolation grid" method presented in ref. [29] with the parameters published in ref. [33], which give sufficient precision (see section 3). For the sake of completeness, the following summarises the interpolation algorithm.

This method first maps $\left(x_{1}, x_{2}, Q^{2}\right) \mapsto\left(y_{1}, y_{2}, \tau\right)$, with

$$
y(x)=5(1-x)-\log x, \quad \tau\left(Q^{2}\right)=\log \log \frac{Q^{2}}{(0.25 \mathrm{GeV})^{2}} .
$$

The function $y(x)$ maps events with large $x$ effectively linearly and small $x$ effectively logarithmically onto $y$. This reflects our knowledge of PDFs, which behave differently in those two regions, and thereby increases the precision of the interpolation. For the convolution of a grid with a PDF set also the inverse functions are needed, which are

$$
x(y)=\frac{1}{5} \mathrm{~W}_{0}(5 \exp (5-y)), \quad Q^{2}(\tau)=(0.25 \mathrm{GeV})^{2} \exp (\exp (\tau)),
$$

where $\mathrm{W}_{0}(x)$ is (the principle branch of) the Lambert $\mathrm{W}$ function or product logarithm, which satisfies the relation $\mathrm{W}(x) \exp (\mathrm{W}(x))=x$.

Following ref. [29] (see eq. (17) therein), we furthermore divide the weights, before filling them into the grid, by the function

$$
\omega\left(x_{1}, x_{2}\right)=\left(\frac{\sqrt{x_{1}}}{1-0.99 x_{1}}\right)^{3}\left(\frac{\sqrt{x_{2}}}{1-0.99 x_{2}}\right)^{3} .
$$

This flattens the interpolated function in the region $x \rightarrow 1$, where the PDFs are small and tend towards zero, and enhances the function in the small- $x$ region. The effect of this step is an improvement of the precision that depends on the initial states and the process, but it can be as large as a factor of 10 to 100 (one or two more correct digits compared to the $\mathrm{MC}$ result). Before performing a convolution this step is inverted by simply multiplying the interpolated grid values with $\omega\left(x_{1}, x_{2}\right)$.

The final step is filling the weights into the grid, which maps the variables $\left(y_{1}, y_{2}, \tau\right)$ onto the 3-dimensional Lagrange-interpolation grid with $N_{y}=50$ points in each $y$ direction and $N_{\tau}=30$ points in the $\tau$ direction. The interpolation orders $s_{y}$ and $s_{\tau}$ are 3 for each dimension, and only the subspace $\left[2 \times 10^{-7}, 1\right] \times\left[2 \times 10^{-7}, 1\right] \times\left[10^{2}, 10^{6}\right] \subset H^{3}$ is mapped.

\footnotetext{
${ }^{3}$ In ref. [33] the upper limit for $Q$ is given as $3162 \mathrm{GeV}$ (which corresponds to $Q_{\max }^{2} \approx 10^{7} \mathrm{GeV}$ ), but in the code we found the value $Q_{\max }^{2}=10^{6} \mathrm{GeV}^{2}$.
} 


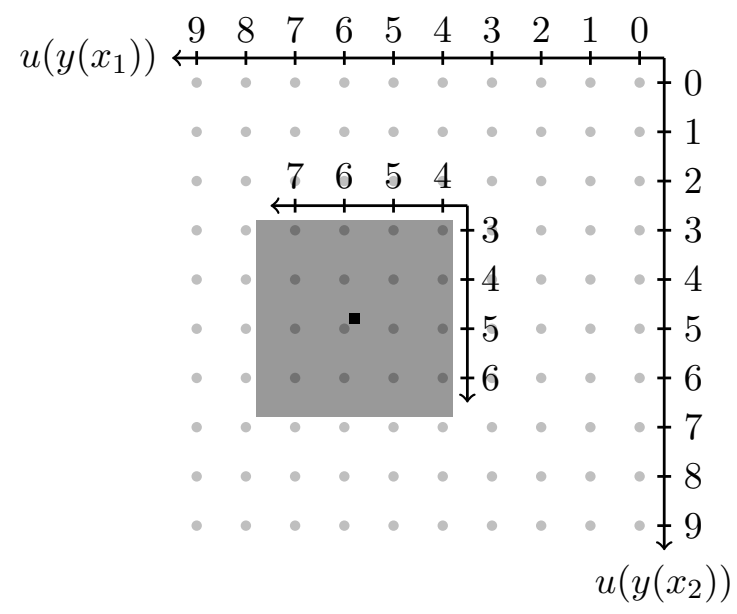

\begin{tabular}{|ll|}
\hline$u_{1} / u_{2}$ & $x_{1} / x_{2}$ \\
\hline 0 & 1.00 \\
1 & $6.36 \times 10^{-1}$ \\
2 & $3.20 \times 10^{-1}$ \\
3 & $9.96 \times 10^{-2}$ \\
4 & $1.57 \times 10^{-2}$ \\
5 & $1.74 \times 10^{-3}$ \\
6 & $1.81 \times 10^{-4}$ \\
7 & $1.87 \times 10^{-5}$ \\
8 & $1.93 \times 10^{-6}$ \\
9 & $2.00 \times 10^{-7}$ \\
\hline
\end{tabular}

Figure 1. Example of a 2-dimensional $10 \times 10$ grid, which is being filled at the location marked with the small black square at $(5.8,4.8)$. Each side of the grey square starting at $k_{i}=4$ and $k_{j}=3$ has a length of $N_{y}+1=4$. This square marks the grid values (grey dots) that are being updated using eq. (2.14). The table on the right-hand side gives the parton-momentum fractions for each grid point according to eq. (2.11). Note that for $u \in[0,3]$ the values are roughly linearly distributed, then logarithmically.

To illustrate the filling step we give an example in figure 1, where, for simplicity, we have chosen a static scale, so that we do not need to interpolate in the $\tau$ direction, and where we also limited the number of grid points to $N_{y}=10$. Each grid point has a numerical value $a_{i, j}$ associated, and the set of all numerical values $\left\{a_{i, j}\right\}$ for all grid indices $(i, j) \in$ $\left[0, N_{y}\right) \times\left[0, N_{y}\right)$ constitute "the grid". Inserting a specific weight $W=w\left(x_{1}, x_{2}\right) / \omega\left(x_{1}, x_{2}\right)$ into the grid is shown in figure 1 as a small black square, inside the larger grey one. We have defined the grid points at specific positions, but the points given by the $\mathrm{MC}$ will land somewhere between them. The interpolation order $s_{y}$ then defines a square with length $s_{y}+1$ around its centre $\left(u\left(y\left(x_{1}\right)\right), u\left(y\left(x_{2}\right)\right)\right)$, given by the MC. All grid points with indices $(i, j)$ covered by the grey square are then updated according to the following formula,

$$
a_{i, j} \leftarrow a_{i, j}+I_{i}\left(u\left(y\left(x_{1}\right)\right)\right) I_{j}\left(u\left(y\left(x_{2}\right)\right)\right) W\left(x_{1}, x_{2}\right) / \omega\left(x_{1}, x_{2}\right),
$$

with the Lagrange basis functions,

$$
I_{i}(u)=\prod_{\substack{k=k_{i} \\ k \neq i}}^{k_{i}+s_{y}} \frac{u-k}{i-k},
$$

where the product runs over all indices of the grid points covered by the grey square in figure 1 , starting from the smallest index in the square, $k_{i}$ and $k_{j}$. Finally, we remap

$$
u(y)=\frac{y-y_{\min }}{\Delta y},
$$

using $y_{\min }=y\left(x_{\max }\right)$ and $y_{\max }=y\left(x_{\min }\right)$ and the grid spacing $\Delta y=\left(y_{\max }-y_{\min }\right) /\left(N_{y}-1\right)$, so that the integer part of $u(y)$ gives the grid point index, e.g. $u\left(y_{\min }\right)=0$ and $u\left(y_{\max }\right)=$ $N_{y}-1$, and the fractional part of $u(y)$ gives the relative location between the nearest grid points. 


\subsection{Perturbative orders}

In section 2.2 we labelled the different perturbative orders using the indices $k, l, m, n$; their values are process specific. However, in general we define as leading order (LO) the set of contributions for all possible initial states $a b$ that lead to the same final state $X$, for which the sum of the coupling exponents in eq. (2.1) is smallest, i.e. $k+l=p$, where $p=\min (k+l)$. This number is usually determined by the number of external particles. For many processes there is only one contribution at LO (in terms of $k$ and $l$ ), but this is not true in general. Indeed, when a process has multiple quark lines, colourless (photons, ....) and coloured particles (gluons, ... ) can be exchanged between them, making it possible to have more than one combination of $\alpha_{\mathrm{s}}$ and $\alpha$. A typical example at the LHC is (on-shell) top-pair production, which has three different contributions at LO: $\mathcal{O}\left(\alpha^{2}\right), \mathcal{O}\left(\alpha_{\mathrm{s}} \alpha\right)$, and $\mathcal{O}\left(\alpha^{2}\right)$. Each contribution receives a higher-order correction with an additional power of $\alpha_{\mathrm{s}}$ or $\alpha$, which in general leads to at least two next-to-leading order (NLO) corrections. The correction with the largest power in $\alpha_{\mathrm{S}}$ can be unambiguously called "the" QCD correction, and the one with the largest power in $\alpha$ "the" EW correction. All remaining corrections are of combined type, meaning that, in general, they cannot be attributed to either one of strong or electroweak origin. However, for the sake of simplicity and with a slight abuse of notation, it is customary to call NLO QCD (EW) corrections those corrections of order $\alpha_{\mathrm{s}}$ $(\alpha)$ times the couplings of the LO contribution with the largest power of $\alpha_{\mathrm{s}}$. In the example above, NLO QCD and EW corrections to top pair production will denote respectively those at $\mathcal{O}\left(\alpha_{\mathrm{s}}^{3}\right)$ and $\mathcal{O}\left(\alpha_{\mathrm{s}}^{2} \alpha\right)$. In this paper, in particular when discussing results in section 3, we will explicitly list the orders that are considered in the cross section at LO, NLO QCD and NLO QCD+EW accuracy.

Due to the typical sizes of the couplings $\alpha_{\mathrm{s}}^{2} \sim \alpha$, it is naively expected that within the same order, i.e. for fixed $k+l$, terms with larger powers $\alpha_{\mathrm{s}}^{k}$ dominate over those with smaller powers (and larger powers of $\alpha^{l}$ ). In practice, however, this naive expectation is not always true due to dynamic effects. Some examples are vector-boson scattering processes [42, 43], top-pair production with a $\mathrm{W}$ boson and four-top production [44], and Higgs production with a bottom-pair [45].

\subsubsection{Example: Drell-Yan lepton-pair production at the LHC}

To give an example of eq. (2.1), the following shows Drell-Yan lepton-pair production up to terms at NLO (with some arguments suppressed for the phase-space weights):

$$
\begin{aligned}
\sigma_{a b}= & \alpha^{2} W_{a b}^{(0,2,0,0)} \\
& +\alpha_{\mathrm{s}}\left(\xi_{\mathrm{R}}^{2} Q^{2}\right) \alpha^{2} W_{a b}^{(1,2,0,0)}\left(Q^{2}\right)+\alpha_{\mathrm{s}}\left(\xi_{\mathrm{R}}^{2} Q^{2}\right) \log \left(\xi_{\mathrm{F}}^{2}\right) \alpha^{2} W_{a b}^{(1,2,0,1)} \\
& +\alpha^{3} W_{a b}^{(0,3,0,0)}\left(Q^{2}\right)+\log \left(\xi_{\mathrm{F}}^{2}\right) \alpha^{3} W_{a b}^{(0,3,0,1)} .
\end{aligned}
$$

The term in the first line is the LO term, the second line shows the NLO QCD correction, and the third line the NLO EW correction. Note that all terms depend on the renormalisation scale only indirectly through $\alpha_{\mathrm{s}}$, because 1) higher-order terms in $\alpha$ never generate a renormalisation scale dependence (in the $\alpha$ schemes that are valid according to section 2.2) 
and 2) higher-order QCD corrections only introduce an explicit renormalisation scale dependence in counterterms with vertices with more than two gluons. At NLO these terms are not present for this process so that terms proportional to $\log \left(\xi_{\mathrm{R}}^{2}\right)$ vanish. Both NLOs, however, have contributions from a collinear counterterm that depends on the factorisation scale. Since this process has a single LO, combined QCD-EW corrections first appear at next-to-next-to-leading order (NNLO), which include the QCD correction at $\mathcal{O}\left(\alpha_{\mathrm{s}}^{2} \alpha^{2}\right)$, the EW correction $\mathcal{O}\left(\alpha^{4}\right)$, and a single combined correction at $\mathcal{O}\left(\alpha_{\mathrm{s}} \alpha^{3}\right)$.

Note that all initial states have to be taken into account that lead to the same final state. This includes the photon-photon initial state, which appears already at LO. In the corresponding Feynman diagrams all particles are colourless, so that the photon-photon initiated contributions only receive EW corrections. The EW corrections also introduce quark-photon contributions, in analogy of QCD corrections introducing quark-gluon contributions.

\section{Validation and interpretation of PineAPPL grids}

In this section we demonstrate the capabilities of the PINEAPPL library by interfacing it to MG5_AMC, and by computing fast-interpolation grids, accurate to NLO QCD and $\mathrm{NLO}$ QCD+EW, for a common set of LHC processes in which EW corrections may sizeably affect the accuracy of the theoretical predictions. In order to consider realistic kinematics for these processes, we rely on a representative set of LHC measurements. Our aim is twofold. First, we want to validate the results obtained with PINEAPPL; second, we want to assess the impact of the EW corrections for the specific experimental setups. We describe the settings employed for the computations, the corresponding results for each process, and possible implications for the determination of PDFs. It is worth to mention that, in those phase-space regions where both NLO QCD and EW corrections are sizeable, the NNLO correction at $\mathcal{O}\left(\alpha_{\mathrm{s}} \alpha\right)$ is also expected to be important, and has either to be included, when it is known exactly or at least in some approximate way, or to be accounted for as an extra uncertainty. We leave this aspect to future studies.

\subsection{Computational settings}

MG5_AMC makes it possible to compute predictions including NLO QCD and EW corrections for arbitrary processes in an automated manner. It employs the FKS subtraction scheme [38, 39] as automated in MADFKS [46, 47] to deal with IR singularities. Oneloop amplitudes are computed by MADLOOP [48], which employs different numerical techniques [49-54] implemented in the corresponding computer libraries [55-58]. Matching with parton showers is available only for pure-QCD corrections via the MC@NLO method [59], and will not be employed in the following.

We generate each process by means of the Universal FeynRules Output (UFO) [60] model loop_qcd_qed_sm_Gmu, included as standard in MG5_AMC. It contains the UV and $R_{2}$ counterterms relevant to NLO QCD and EW corrections, the latter in the $\bar{G}_{\mu}$ scheme. The model features five massless quark flavours, sets the CKM matrix equal to the identity, and is compatible with the usage of the complex mass (CM) scheme [61, 62] for all 
massive particles, see ref. [11] for details. We use this scheme for all processes that involve only massless particles in the final state. The photon is always considered as part of the proton in the initial state and of any hadronic jet produced in the final state: in particular, photon-induced (PI) contributions are consistently included at LO and NLO. ${ }^{4}$ We use a PDF set that contains a photon PDF, namely NNPDF31_nlo_as_0118_luxqed [21]. We evaluate the PDF uncertainty associated to the theoretical predictions a posteriori, that is, we convolve the fast-interpolation grid generated with PINEAPPL with each member in the PDF set, and we compute the associated standard deviation. Monte Carlo weights are stored as Lagrange-interpolation grids.

The central values of the renormalisation and factorisation scales, $\mu_{\mathrm{R}}$ and $\mu_{\mathrm{F}}$, are chosen in a process-specific way, as discussed in section 3.2. In order to estimate the missing higher-order uncertainty, we allow the events to be reweighted on-the-fly in the Monte Carlo generation upon scale variations, with the technique presented in ref. [63]. To this purpose, we use the default MG5_AMC implementation, whereby the factorisation and renormalisation scales are varied down to a factor $1 / 2$ and up to a factor 2 , and the envelope from the nine-point scale variations is constructed, see equation (2.5). However, we note that PINEAPPL allows the user to determine the envelope with any point prescription, see appendix A.1 for an example.

The values of the relevant physical parameters are chosen as

$$
\begin{aligned}
& M_{\mathrm{W}}=80.419 \mathrm{GeV}, \quad \Gamma_{\mathrm{W}}=2.09291 \mathrm{GeV}, \\
& m_{\mathrm{t}}=172.5 \mathrm{GeV} \text {, } \\
& M_{\mathrm{Z}}=91.176 \mathrm{GeV}, \quad \Gamma_{\mathrm{Z}}=2.49877 \mathrm{GeV}, \\
& G_{\mu}=1.16639 \times 10^{-5} \mathrm{GeV}^{-2}, \\
& M_{\mathrm{H}}=125 \mathrm{GeV}, \quad \Gamma_{\mathrm{H}}=4.074680 \times 10^{-3} \mathrm{GeV},
\end{aligned}
$$

where $M_{\mathrm{Z}}, M_{\mathrm{W}}, M_{\mathrm{H}}, m_{\mathrm{t}}$ are the values of the Z-, W-, Higgs-boson and top-quark masses, respectively, $\Gamma_{\mathrm{Z}}, \Gamma_{\mathrm{W}}, \Gamma_{\mathrm{H}}$ are the widths of the $\mathrm{Z}, \mathrm{W}$ and Higgs bosons, and $G_{\mu}$ is the value of the Fermi coupling. The value of the strong coupling is chosen consistently with the PDF set, $\alpha_{\mathrm{s}}\left(M_{\mathrm{Z}}\right)=0.118$.

The definition of observables and cuts is process-specific, and it follows the corresponding experimental measurements, see section 3.2. When relevant, final-state photons and massless charged fermions (leptons and light quarks) are recombined together if they satisfy the condition $\Delta R_{f \gamma}<0.1$, where $\Delta R_{f \gamma}$ is the fermion-photon distance. In this case the sum of their momenta is assigned to the charged fermion, and the photon is removed from the event. Kinematic observables and cuts are defined starting from recombined momenta. If we were interested also in jet-related observables, photons surviving the recombination would have to be clustered together with coloured partons. ${ }^{5}$ Finally, although contributions corresponding to the radiation of a heavy boson are formally of the same perturbative order of the EW corrections, they are not included in our computations. In fact, while nothing prevents one to include these contributions a posteriori, as they are finite, their impact

\footnotetext{
${ }^{4}$ We employ the $\bar{G}_{\mu}$ scheme also for the QED coupling entering vertices involving initial-state photons, see section 4.3 .3 of ref. [12].

${ }^{5}$ For issues related to the definition of jets in presence of $\mathrm{EW}$ corrections, in particular about the fragmentation of partons into photons and vice-versa, see refs. [64-66].
} 
is either smaller than the one of "standard" EW corrections, or anyway negligible with respect to the total cross section (see refs. [67-69] for some process-specific cases).

\subsection{Results for specific processes and measurements}

We focus on the following three processes: Drell-Yan lepton-pair production, top-quark pair production, and Z-boson (lepton-pair) production with non-zero transverse momentum in proton-proton collisions. These are some of the most commonly and most precisely measured processes at the LHC, which are widely studied to test the SM and/or search for new physics. We therefore expect them to allow us to clearly show the benefit of being able to make fast and reliable theoretical predictions accurate to NLO QCD+EW with PINEAPPL. In the following, we shall present the experimental measurements, the process-specific settings, and the phenomenological results for each of these processes.

When presenting the results, for each of the processes and measurements considered, we compute differential cross sections for the observables defined in the experimental analyses in two different ways: directly, by means of MG5_AMC, and a posteriori, by convolving the fast-interpolation grid produced by PINEAPPL with the PDF set specified in section 3.1. We refer to the first result as the MC result, and to the second as the PINEAPPL result. We repeat the computation for theories accurate to NLO QCD and to NLO QCD+EW, respectively. The corresponding orders of the strong and EW couplings that we consider are specified for each process. In each case, we determine the PDF uncertainty (coming from the PDF ensemble), the scale uncertainty (coming from variations of the factorisation and renormalisation scales), and the Monte Carlo uncertainty (coming from the finite number of events generated). In this last respect, we consider by default high-statistics computations, whereby we require a relative Monte Carlo precision of $0.1 \%$ on the integrated cross section. While this choice does not affect the validation of the PINEAPPL result against the MC result, it ensures that the statistical uncertainty of the computation remains negligible in comparison to the PDF and scale uncertainties, as we will explicitly demonstrate. This is a desirable feature to correctly interpret the size of the EW corrections. An example that validates the PINEAPPL result in the case of a low-statistic run is nevertheless provided in appendix C.

Our goal is indeed twofold. On the one hand, we aim to validate the interpolation grids generated with PINEAPPL: to this purpose we shall verify that the MC and the PINEAPPL results are identical up to numerical inaccuracies due to the grid interpolation. This equivalence must hold for any choice of renormalisation and factorisation scale and should not depend on the MC uncertainty of the binned cross section. On the other hand, we aim to study the size of the EW corrections, in particular with respect to the kinematics of each process, and to three kinds of uncertainties: the PDF uncertainty, the scale uncertainty, and the uncertainty of the experimental data.

We present these comparisons in figures $2,3,4$, and 5 . The format of the plots is the same across all figures. The first panel displays the relative difference (in per mille) between the PINEAPPL and the MC results for the central scale choice and upper/lower edges of the scale-uncertainty envelope, for theories accurate to both NLO QCD and NLO QCD+EW. The following three panels present the theoretical predictions, accurate to 
either NLO QCD or NLO QCD+EW, always normalised to the former; on top of the theoretical predictions, the PDF uncertainty, the scale uncertainty and the Monte Carlo uncertainty are displayed in turn. The relative uncertainty of the experimental data is also shown for comparison. We shall now discuss the results for each process and data set.

\subsubsection{Drell-Yan lepton pair production}

Experimental measurements and process features. We select the single-differential invariant mass distribution of the lepton pair, $M_{\ell \bar{\ell}}$, measured by the ATLAS experiment at a centre-of-mass energy of $7 \mathrm{TeV}$ in the high-mass region $\left(M_{\ell \bar{\ell}}>116 \mathrm{GeV}\right)$ [70]. We also select the single-differential rapidity distribution, $y_{\ell \bar{\ell}}$, in slices of the invariant mass of the lepton pair, $M_{\ell \bar{\ell}}$, measured by the CMS experiment at a centre-of-mass energy of $7 \mathrm{TeV}$ [71]. These measurements are currently included as standard in the NNPDF3.1 [14] and MMHT2014 [13] PDF sets, although with appropriate kinematic cuts that remove the bins at the largest values of invariant mass, where EW corrections become sizeable. As explained in section 2.3.1, the process has a single LO, $\mathcal{O}\left(\alpha^{2}\right)$; at NLO, the QCD contribution is $\mathcal{O}\left(\alpha_{\mathrm{s}} \alpha^{2}\right)$, while the EW contribution is $\mathcal{O}\left(\alpha^{3}\right)$. Our NLO QCD computation includes the $\mathcal{O}\left(\alpha^{2}\right)$ and $\mathcal{O}\left(\alpha_{\mathrm{s}} \alpha^{2}\right)$ contributions, while our NLO QCD+EW computation includes the $\mathcal{O}\left(\alpha^{2}\right), \mathcal{O}\left(\alpha_{\mathrm{s}} \alpha^{2}\right)$ and $\mathcal{O}\left(\alpha^{3}\right)$ contributions. Combined QCD-EW corrections occur only at NNLO [72], and are therefore not considered here. EW corrections for this process were computed in refs. [11,73-75] (see also [76] and references therein). The process receives contributions from 13 (35) parton luminosities at NLO QCD (NLO QCD+EW), see appendix B for details.

Process-specific settings. We use a fixed value for the renormalisation and factorisation scales $\mu_{\mathrm{R}}=\mu_{\mathrm{F}}=M_{\mathrm{Z}}$, where $M_{\mathrm{Z}}$ is the mass of the $\mathrm{Z}$ boson, for the ATLAS measurement, and the scale $\mu_{\mathrm{R}}=\mu_{\mathrm{F}}=M_{\ell \bar{\ell}}$, where $M_{\ell \bar{\ell}}$ is the central value of each invariant mass slice, for the CMS one. In the case of ATLAS, we require $p_{\mathrm{T}}^{\ell}>25 \mathrm{GeV},\left|\eta_{\ell}\right|<2.5$ and $116 \mathrm{GeV}<M_{\ell \bar{\ell}}<1500 \mathrm{GeV}$ for the transverse momentum and the pseudorapidity of each lepton and for the invariant mass of the lepton pair, respectively. Conversely, in the case of CMS, we require $p_{\mathrm{T}}^{\ell_{1}}>14 \mathrm{GeV}, p_{\mathrm{T}}^{\ell_{2}}>9 \mathrm{GeV},\left|\eta_{\ell}\right|<2.4,\left|y_{\ell \bar{\ell}}\right|<2.4$ and $20 \mathrm{GeV}<M_{\ell \bar{\ell}}<1500 \mathrm{GeV}$ for the transverse momentum and the pseudorapidity of each lepton, and for the rapidity and the invariant mass of the lepton pair.

Numerical results. We first consider the single-differential measurement of a leptonpair for large invariant masses performed by the ATLAS experiment at $7 \mathrm{TeV}$. From figure 2 we immediately observe that the validation of the PINEAPPL result against the MC result is successful. The relative difference between the two is of the order of $0.1 \%$ at most, with negligible fluctuations across different invariant mass bins. The agreement is similarly good irrespective of the perturbative accuracy of the theory (NLO QCD or NLO QCD+EW) or of the scale choice. As explicitly demonstrated in appendix $\mathrm{C}$, the good agreement is also independent from the numerical precision of the Monte Carlo run.

The measurement is mainly driven by $q \bar{q}$ scattering: specifically, the leading (nextto-leading) contribution comes from a $u \bar{u} / c \bar{c}(d \bar{d} / s \bar{s})$ parton luminosity, which accounts for about $55 \%(49 \%)$ of the cross section for the lowest invariant mass bins, and $68 \%$ 


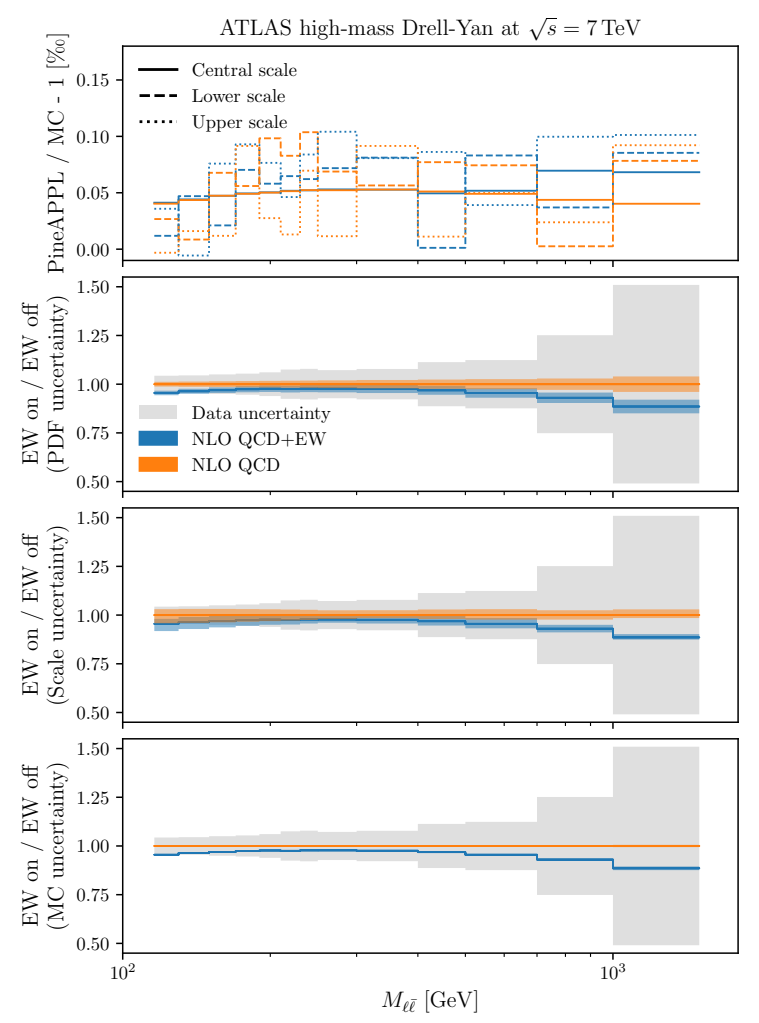

Figure 2. Validation and test of the PINEAPPL grid for the ATLAS Drell-Yan lepton pair measurement in the high-mass region at a centre-of-mass energy of $7 \mathrm{TeV}$ [70]. The first panel displays the relative difference (in per mille) between the PINEAPPL and the MC results for the central, upper and lower scale choices, for theories accurate to both NLO QCD and NLO QCD+EW. The second, third and fourth panels present the theoretical predictions, accurate to either NLO $\mathrm{QCD}$ or NLO QCD+EW, always normalised to the former; on top of the theoretical predictions, the PDF uncertainty, the scale uncertainty and the Monte Carlo uncertainty are displayed in turn. The relative uncertainty of the experimental data is also shown for comparison.

$(22 \%)$ for the largest invariant mass bins. ${ }^{6}$ The PI contribution raises from about $1.3 \%$ in the lowest bin to about $3.6 \%$ in the highest bin. ${ }^{7}$ Overall, the EW corrections range between $-5 \%$ around $M_{\ell \bar{\ell}} \lesssim 150 \mathrm{GeV},-2 \%$ to $-3 \%$ for intermediate invariant mass values, $150 \mathrm{GeV} \lesssim M_{\ell \bar{\ell}} \lesssim 700 \mathrm{GeV}$, and $-6 \%$ to $-10 \%$ for the largest invariant mass bin, $M_{\ell \bar{\ell}}>1000 \mathrm{GeV}$, see figure 2. For this reason, the data points with $M_{\ell \bar{\ell}}>210 \mathrm{GeV}$ were not included in the NNPDF3.1 analysis [14].

The NLO QCD + EW corrections always lead to a reduction of the cross section in comparison to the NLO QCD prediction. The size of this shift is comparable to the data uncertainty at small values of $M_{\ell \bar{\ell}}$, and rapidly becomes negligible with respect to it as the value of the invariant mass increases and the data uncertainty blows up. This fact suggests

\footnotetext{
${ }^{6}$ The size of these contributions may depend on the input PDF set. Here and in the following, we always quote results obtained from the NNPDF31_nlo_as_0118_luxqed PDF set.

${ }^{7}$ For the MRST2004qed PDF set [16], which is used to subtract PI contributions (see section 4), the value in the highest bin is roughly twice as large, $6.9 \%$ (also in absolute numbers).
} 
a couple of observations in light of the inclusion of EW corrections in a fit of PDFs. First, the description of the more precise bins in the low invariant mass range is likely to change, and will possibly become more accurate should the inclusion of EW corrections improve the data/theory agreement. Second, the kinematic cut that excludes any data point at large $M_{\ell \bar{\ell}}$ can be safely removed: any shift in the predictions induced by the more accurate NLO QCD+EW theory is likely to be easily accommodated by the large data uncertainty. However, due to the increased Run-II LHC luminosity, data will become more precise.

In comparison to the PDF uncertainty, the size of the EW corrections is always larger, especially at the boundaries of the distribution. This fact suggests that, once included in a global fit, EW corrections will make PDFs more accurate. In comparison to the scale uncertainty, the size of the EW correction is similar, except for the four bins at the largest invariant mass, where the latter is significantly larger than the former. This fact suggests that the impact of NNLO QCD corrections [77-81] is comparable to the one of NLO $\mathrm{QCD}+\mathrm{EW}$, except at very large values of the invariant mass, where the EW correction still dominates. This result stresses the need to include the EW corrections in order to obtain an accurate description of the large invariant mass bins. Finally, the Monte Carlo uncertainty remains negligible in comparison to the data, PDF and scale uncertainties, and to the size of the EW correction. Our conclusions should therefore not be affected by a generation of too few Monte Carlo events.

We then turn our attention to the double-differential measurement performed by the CMS experiment at $7 \mathrm{TeV}$. For illustrative purposes, we report only four out of the six invariant mass bins, respectively below the Z-boson mass peak, $45 \mathrm{GeV}<M_{\ell \bar{\ell}}<60 \mathrm{GeV}$, on the Z-boson mass peak, $60 \mathrm{GeV}<M_{\ell \bar{\ell}}<120 \mathrm{GeV}$, above the mass peak, $120 \mathrm{GeV}<M_{\ell \bar{\ell}}<$ $200 \mathrm{GeV}$, and at very high invariant masses, $200 \mathrm{GeV}<M_{\ell \bar{\ell}}<1500 \mathrm{GeV}$, see figure 3 . Analogous plots for the remaining low invariant mass bins are collected in appendix C. From figure 3, first of all we validate the PINEAPPL result: its relative difference with respect to the MC result is always below a fraction of per mille, again irrespective of the accuracy of the theory, of the choice of scale, and of the kinematic bin considered.

As in the case of the ATLAS high-mass Drell-Yan measurement, the CMS measurement is also dominated by $q \bar{q}$ scattering. The leading (next-to-leading) contribution to the $45 \mathrm{GeV}<M_{\ell \bar{\ell}}<60 \mathrm{GeV}$ invariant mass bin comes from the $\mathrm{u} \overline{\mathrm{u}} / \mathrm{c} \overline{\mathrm{c}}(\mathrm{d} \overline{\mathrm{d}} / \mathrm{s} \overline{\mathrm{s}})$ parton luminosity, which accounts for about $70 \%(22 \%)$ of the double differential cross section, with small fluctuations across the rapidity range. The PI contribution decreases from about $4 \%$ at zero rapidity to $1.5 \%$ in the largest rapidity bin. The situation is slightly different in the $60 \mathrm{GeV}<M_{\ell \bar{\ell}}<120 \mathrm{GeV}$ invariant mass bin, where the leading (next-to-leading) contribution comes instead from the $\mathrm{d} \overline{\mathrm{d}} / \mathrm{s} \overline{\mathrm{s}}(\mathrm{u} \overline{\mathrm{u}} / \mathrm{c} \overline{\mathrm{c}})$ parton luminosity, which accounts for about $60 \%$ (44\%) of the double differential cross section at small rapidities, and for about $56 \%$ (50\%) at large rapidities. In the remaining two invariant mass bins, the leading (next-to-leading) contribution comes again from the $u \bar{u} / c \bar{c}(d \bar{d} / s \bar{s})$ parton luminosity, which accounts for about $69 \%$ to $95 \%$ (38\% to $30 \%)$ and $57 \%$ to $70 \%$ (48\% to $34 \%)$ of the cross section, respectively for $120 \mathrm{GeV}<M_{\ell \bar{\ell}}<200 \mathrm{GeV}$ and $200 \mathrm{GeV}<M_{\ell \bar{\ell}}<1500 \mathrm{GeV}$ in the corresponding rapidity intervals; PI contributions range between $3.7 \%$ to $0.6 \%$ and $7.3 \%$ to $1.6 \%$ in the two invariant mass bins, respectively, for increasing rapidity. 

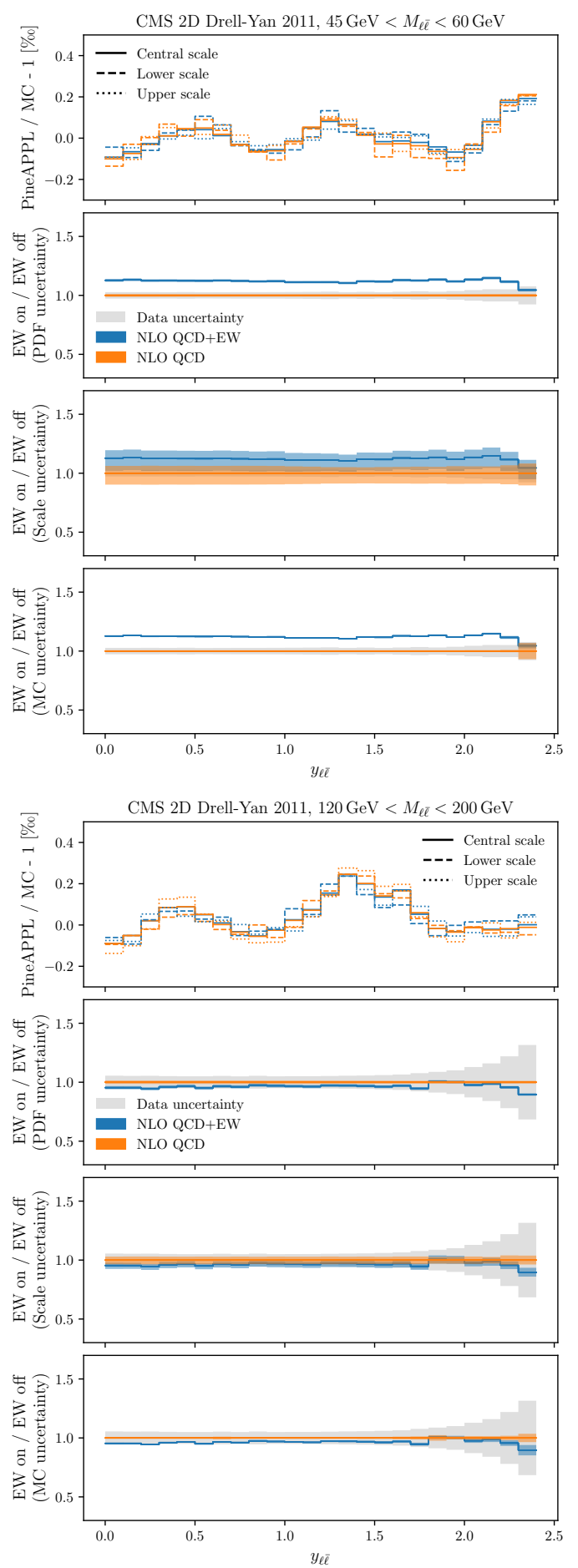
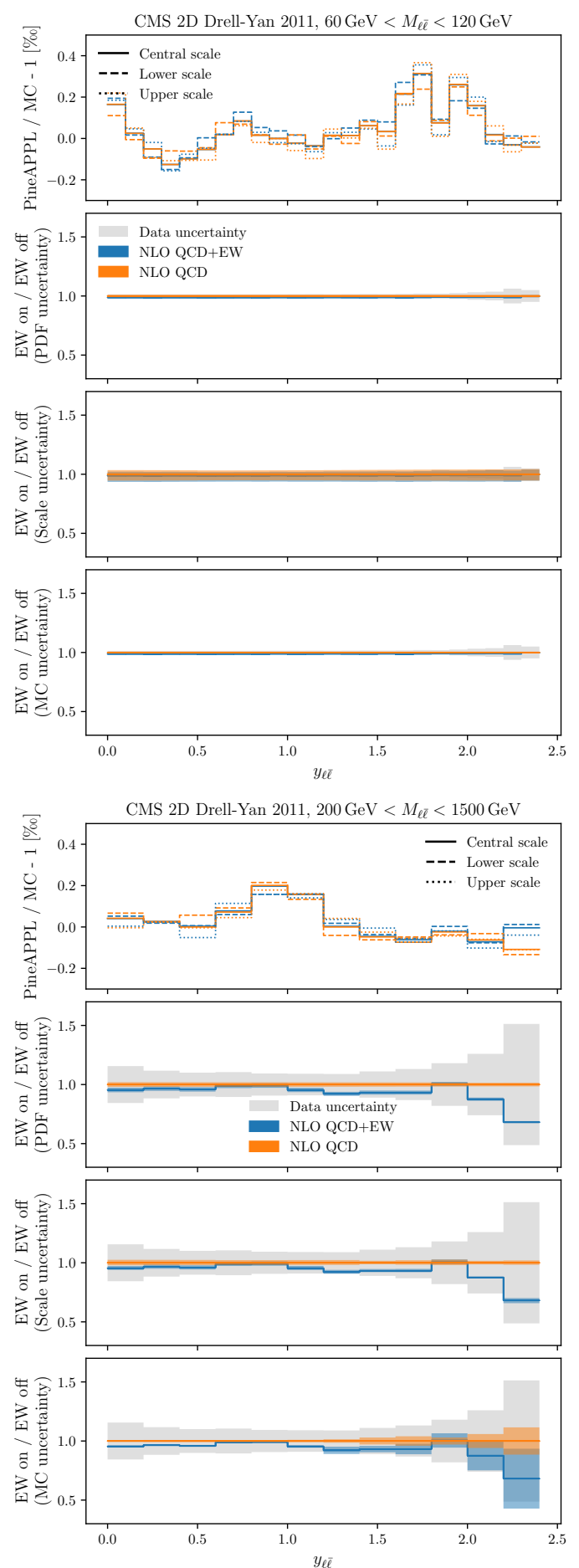

Figure 3. Same as figure 2, but for the CMS double-differential Drell-Yan lepton pair measurement at a centre-of-mass energy of $7 \mathrm{TeV}$ [71]. Displayed are only four of the six invariant mass bins available, respectively below the Z-boson mass peak, $45 \mathrm{GeV}<M_{\ell \bar{\ell}}<60 \mathrm{GeV}$, on the Z-boson mass peak, $60 \mathrm{GeV}<M_{\ell \bar{\ell}}<120 \mathrm{GeV}$, above the mass peak, $120 \mathrm{GeV}<M_{\ell \bar{\ell}}<200 \mathrm{GeV}$, and at very high invariant masses, $200 \mathrm{GeV}<M_{\ell \bar{\ell}}<1500 \mathrm{GeV}$. Results for the slices $45 \mathrm{GeV}<M_{\bar{\ell} \bar{\ell}}<60 \mathrm{GeV}$ and $45 \mathrm{GeV}<M_{\ell \bar{\ell}}<60 \mathrm{GeV}$ can be found in figure 8 . 
The way in which NLO QCD+EW corrections affect the theoretical prediction for the double differential cross section (with respect to its counterpart accurate to NLO QCD) depends on the invariant mass bin. In the $45 \mathrm{GeV}<M_{\ell \bar{\ell}}<60 \mathrm{GeV}$ region, they enhance the value of the cross section by about $11 \%$ across all the rapidity range. This is mostly due to photon-radiation effects on events with $M_{\ell \bar{\ell}} \simeq M_{\mathrm{Z}}$ at the Born, for which the invariant mass is shifted to lower values. In the $60 \mathrm{GeV}<M_{\bar{\ell} \bar{\ell}}<120 \mathrm{GeV}$ region, EW corrections suppress the value of the cross section by about $2 \%$, again across all the rapidity range; in the $120 \mathrm{GeV}<M_{\ell \bar{\ell}}<200 \mathrm{GeV}$ bin, the suppression is around $4 \%$ to $5 \%$; and in the $200 \mathrm{GeV}<M_{\ell \bar{\ell}}<1500 \mathrm{GeV}$ bin, the suppression increases further to about $6 \%$ to $7 \%$ for rapidities $y_{\ell \bar{\ell}}<2.0$. For this reason, for instance, the data points with $M_{\ell \bar{\ell}}>200 \mathrm{GeV}$ and $y_{\ell \bar{\ell}}>2.2$ were not included in the NNPDF3.1 analysis [14].

In general, the size of the EW corrections is comparable to or slightly larger than the data uncertainty, except for the invariant mass bin $45 \mathrm{GeV}<M_{\ell \bar{\ell}}<60 \mathrm{GeV}$, where the shift due to the EW correction overshoots the data uncertainty by about a factor of ten, and at large rapidities, where the shift due to the EW correction, although it can become large, is always a fraction of the data uncertainty. Because EW effects are subtracted from the data used in PDF fits (see section 4), a good agreement between data and theory is usually achieved without the inclusion of EW corrections. However, as already observed in the case of the ATLAS measurement, should EW corrections be included in a fit of PDFs, the latter are likely to become more accurate: even though the apparent description of the data will not improve, by including the more precisely predicted bins in the low invariant mass range, PDFs will resemble more closely the underlying truth. Furthermore, the kinematic cut that excludes any data point at large invariant mass and/or rapidity can be safely removed.

In comparison to the PDF uncertainty, the size of the EW corrections is always larger. We therefore anticipate that, even if the agreement between the more accurate theory (including EW corrections) and the data will remain the same, the PDFs will however become overall more accurate. In comparison to the scale uncertainty, the size of the EW correction is similar, except on the Z-boson mass peak, $60 \mathrm{GeV}<M_{\ell \bar{\ell}}<120 \mathrm{GeV}$, where the scale uncertainty exceeds the size of the EW correction by about a factor of five. This is due to the choice of invariant mass window around the $\mathrm{Z}$ peak, in which positive and negative EW corrections almost cancel. Finally, the Monte Carlo uncertainty remains negligible in comparison to the data, PDF and scale uncertainties, and to the size of the EW correction, except for a couple of bins at forward/backward rapidity in the highest invariant mass bins. Improving the Monte Carlo precision will require to generate a larger number of events, possibly with cuts that select only the kinematic bins affected by the largest MC uncertainties. If this turned out to be computationally too expensive, it would be desirable to treat this uncertainty as an additional theoretical uncertainty in the PDF fit [82].

\subsubsection{Top-quark pair production}

Experimental measurements and process features. We select the single-differential distribution in either the transverse momentum of the top quark, $p_{\mathrm{T}}^{\mathrm{t}}$, or the invariant mass of the top-quark pair, $m_{\mathrm{t} \overline{\mathrm{t}}}$, measured by the ATLAS and CMS experiments at a centreof-mass energy of $8 \mathrm{TeV}[83,84]$. These measurements have been extensively studied in 
the context of PDF fits in refs. [8, 85-87] (see also refs. [69, 88] for studies related to the photon density) and included by default in the CT18 [15] analysis. Because EW corrections are significantly smaller for distributions differential in the rapidity of either the top quark or the top-quark pair [88], these distributions were preferred for inclusion in the NNPDF3.1 analysis [14]. The process receives pure QCD contributions at LO, $\mathcal{O}\left(\alpha_{\mathrm{s}}^{2}\right)$, and at NLO, $\mathcal{O}\left(\alpha_{\mathrm{s}}^{3}\right)$. These orders make up our NLO QCD computation. The NLO QCD+EW computation includes the $\mathcal{O}\left(\alpha_{\mathrm{s}}^{2}\right)$ and $\mathcal{O}\left(\alpha_{\mathrm{s}}^{3}\right)$ QCD contributions, the LO contribution $\mathcal{O}\left(\alpha_{\mathrm{s}} \alpha\right)$ and the NLO contribution $\mathcal{O}\left(\alpha_{\mathrm{s}}^{2} \alpha\right)$. We do not consider the LO contribution $\mathcal{O}\left(\alpha^{2}\right)$ nor the NLO contributions $\mathcal{O}\left(\alpha_{\mathrm{s}} \alpha^{2}\right)$ and $\mathcal{O}\left(\alpha^{3}\right)$, which have been shown to be negligible $[11,88]$. EW corrections for this process were computed in refs. [11, 69, 8899]. The process receives contributions from 7 (37) parton luminosities at NLO QCD (NLO $\mathrm{QCD}+\mathrm{EW})$, see appendix B for details.

Process-specific settings. We employ the following functional form for the renormalisation and factorisation scales; $\mu_{\mathrm{R}}=\mu_{\mathrm{F}}=\sqrt{m_{\mathrm{t}}^{2}+\left(p_{\mathrm{T}}^{\mathrm{t}}\right)^{2}} / 2$ for the distribution differential in the transverse momentum of the top quark, and $\mu_{\mathrm{R}}=\mu_{\mathrm{F}}=H_{\mathrm{T}} / 4$ for the distribution differential in the invariant mass of the top-quark pair, where $H_{\mathrm{T}}=\sqrt{m_{\mathrm{t}}^{2}+\left(p_{\mathrm{T}}^{\mathrm{t}}\right)^{2}}+\sqrt{m_{\mathrm{t}}^{2}+\left(p_{\mathrm{T}}^{\mathrm{T}}\right)}$, with $m_{\mathrm{t}}, p_{\mathrm{T}}^{\mathrm{t}}$ and $p_{\mathrm{T}}^{\overline{\mathrm{t}}}$ the mass of the top quark and the transverse momenta of the top and antitop quarks, respectively. These choices were demonstrated to maximise the convergence of the perturbative expansion [100]. No cuts are imposed.

Numerical results. In figure 4 we report the distributions differential in the transverse momentum of the top quark, $p_{\mathrm{T}}^{\mathrm{t}}$, and in the invariant mass of the top-quark pair, $m_{\mathrm{t} \overline{\mathrm{t}}}$. Analogous plots for the distributions differential in the rapidity of either the top quark or the top-quark pair are collected in appendix C. From figure 4, we immediately validate the PINEAPPL result: its relative difference with respect to the MC result is at most as large as $0.4 \%$, irrespective of the accuracy of the theory, of the choice of scale and of the distribution considered.

The process receives its leading contribution from the gg channel, which varies between $81 \%$ and $61 \%(76 \%$ and $83 \%)$ of the $p_{\mathrm{T}}^{\mathrm{t}}\left(m_{\mathrm{t} \overline{\mathrm{t}}}\right)$ differential cross section as the value of the transverse momentum of the top quark (the invariant mass of the top-quark pair) increases; the largest PI contribution for this process comes from $\gamma \mathrm{g}$ scattering, which accounts for about $0.5 \%$ to $1 \%(0.5 \%$ to $0.7 \%)$ of the cross section, and is almost entirely (90\%) due to the LO contribution at $\mathcal{O}\left(\alpha_{\mathrm{s}} \alpha\right)$; the contribution from other PI parton luminosities is comparatively negligible. Overall, the EW corrections suppress the $p_{\mathrm{T}}^{\mathrm{t}}\left(m_{\mathrm{t} \overline{\mathrm{t}}}\right)$ distribution by about $0.2 \%$ to $3.5 \%(0.5 \%$ to $0.2 \%)$ for increasing values of $p_{\mathrm{T}}^{\mathrm{t}}\left(m_{\mathrm{t} \overline{\mathrm{t}}}\right)$, except in the first bin of the $p_{\mathrm{T}}^{\mathrm{t}}$ distribution, where they enhance the cross section by about $1 \%$. The size of these shifts, however, remains always significantly smaller than the data uncertainty. ${ }^{8}$ As a consequence, we anticipate that the more accurate NLO QCD+EW theory is likely to be easily accommodated by the large data uncertainty, should the data be fitted with the inclusion of EW corrections.

\footnotetext{
${ }^{8}$ In figure 4 the data uncertainty corresponds to the ATLAS measurement [101]. Similar considerations apply also for the CMS measurement [102].
} 

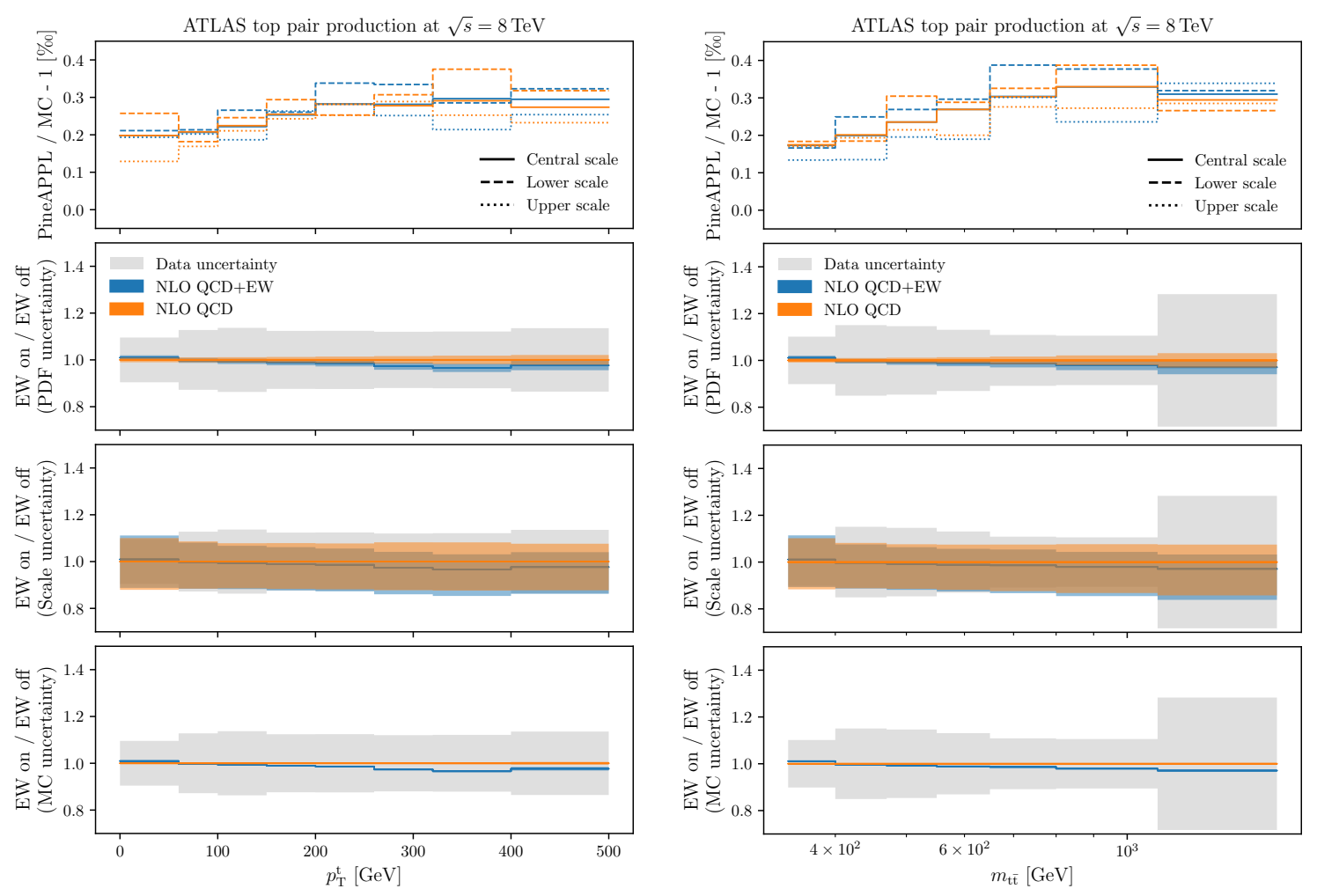

Figure 4. Same as figure 2, but for the ATLAS differential top-quark pair measurement at a centre-of-mass energy of $8 \mathrm{TeV}$ [83]. Displayed are the distributions in the transverse momentum of the top quark $p_{\mathrm{T}}^{\mathrm{t}}$ (left), and in the invariant mass of the top-quark pair $m_{\mathrm{t} \mathrm{t}}$ (right).

The size of the EW correction is comparable to the size of the PDF uncertainty, except at large values of transverse momentum or invariant mass, where the former becomes larger than the latter. This fact suggests that, once included in a global fit, EW corrections can improve the accuracy of the PDFs. In comparison to the scale uncertainty, the size of the EW corrections remains negligible: despite the fact that the choice of factorisation and renormalisation scales have been devised to optimise the convergence of the perturbative expansion, NNLO QCD corrections remain large [103-111], as expected in a process mostly initiated by gluons. Their inclusion is therefore mandatory in a fit of PDFs. Finally, the Monte Carlo statistical uncertainty remains negligible in comparison to the data, PDF and scale uncertainties, and to the size of the EW correction. Our conclusions are therefore not affected by Monte Carlo inefficiencies.

\subsubsection{Z-boson production with non-zero transverse momentum}

Experimental measurements and process features. We select the single-differential transverse momentum distribution of the sum of the two leptons (the "Z boson"), $p_{\mathrm{T}}^{\ell \bar{\ell}}$, measured by the CMS experiment at a centre-of-mass energy of $13 \mathrm{TeV}$ [112]. This measurement, which has not been included in any PDF determination so far, shows very low experimental uncertainties (at the percent level or below). EW corrections are therefore 
expected to be essential to achieve a good description of it, and to constrain accurately the PDFs, together with NNLO QCD corrections, which are already well known [113119]. Analogous measurements, from the ATLAS [101] and CMS [102] experiments at a centre-of-mass energy of $8 \mathrm{TeV}$, were partly included (upon selection of an appropriate kinematic cut that excluded bins with large EW corrections) in a dedicated study [120], in the NNPDF3.1 PDF set [14] and in variants of the CT18 PDF set [15]. In the QCD computation, we consider a single LO contribution $\mathcal{O}\left(\alpha_{\mathrm{S}} \alpha^{2}\right)$ and a single NLO contribution $\mathcal{O}\left(\alpha_{\mathrm{s}}^{2} \alpha^{2}\right)$. In the NLO QCD+EW computation, we supplement these with another LO and NLO, which are $\mathcal{O}\left(\alpha^{3}\right)$ and $\mathcal{O}\left(\alpha_{\mathrm{s}} \alpha^{3}\right)$; contributions of the order $\mathcal{O}\left(\alpha^{4}\right)$ are not considered (see ref. [66]). EW corrections for this process were computed in refs. [11, 121-124]. The process receives contributions from 101 (166) parton luminosities, see appendix B.

Process-specific settings. We choose a dynamic value for the renormalisation and factorisation scales $\mu_{\mathrm{R}}=\mu_{\mathrm{F}}=\sqrt{M_{\ell \bar{\ell}}^{2}+\left(p_{\mathrm{T}}^{\ell \bar{\ell}}\right)^{2}}$, used for example in ref. [117], where $M_{\ell \bar{\ell}}$ is the invariant mass of the lepton pair and $p_{\mathrm{T}}^{\ell \bar{\ell}}$ its transverse momentum. ${ }^{9}$ Consistently with the experimental analysis, we require $p_{\mathrm{T}}^{\ell}>25 \mathrm{GeV},\left|\eta_{\ell}\right|<2.4,\left|M_{\ell \bar{\ell}}-M_{\mathrm{Z}}\right|<15 \mathrm{GeV}$, $\left|y_{\ell \bar{\ell}}\right|<2.4$ and $20 \mathrm{GeV}<p_{\mathrm{T}}^{\ell \bar{\ell}}<1500 \mathrm{GeV}$ for the transverse momentum and pseudorapidity of each lepton, and for the invariant mass, pseudorapidity and transverse momentum of the lepton pair. We finally discard all bins with $p_{\mathrm{T}}^{\ell \bar{\ell}}<20 \mathrm{GeV}$ to avoid a kinematic region where resummation effects are sizeable.

Numerical results. In figure 5 we report the distribution differential in the transverse momentum of the $\mathrm{Z}$ boson. Also in this case, the PINEAPPL result is well validated, as it differs from the MC result by $0.5 \%$ at most. The accuracy of the theory or the choice of scale do not alter this conclusion.

The process receives its leading contribution from qg- and $\bar{q} g$-initiated channels, which account for about $79 \%$ of the cross section, with some variations in the relative contributions from individual quark and antiquark flavours as the transverse momentum of the $\mathrm{Z}$ boson varies; the PI contribution always remain negligible. Overall, the EW corrections suppress the theoretical predictions by about $2 \%$ to $15 \%$ as the transverse momentum of the $\mathrm{Z}$ boson increases. The size of this shift is as large (or slightly larger) as the data uncertainty over the entire range of $p_{\mathrm{T}}^{\bar{\ell}}$. As a consequence, we anticipate the inclusion of EW corrections to be relevant for an accurate fit of this data.

The size of the EW correction is between four and fifteen times larger than the size of the PDF uncertainty: as previously noted in the other cases, this fact suggests that, once included in a PDF fit, EW corrections can improve the accuracy of the PDFs. In comparison to the scale uncertainty, the size of the EW corrections remains negligible at small values of $p_{\mathrm{T}}^{\ell \bar{\ell}}$, roughly $p_{\mathrm{T}}^{\ell \bar{\ell}} \lesssim 400 \mathrm{GeV}$, while it becomes larger than it in the two bins at the largest value of $p_{\mathrm{T}}^{\ell \bar{\ell}}$. In this kinematic region, NLO EW corrections might therefore become even more relevant than NNLO QCD corrections, and should therefore be mandatorily included

\footnotetext{
${ }^{9}$ In the last bin the dynamic scale is $\mu_{\mathrm{R}}=\mu_{\mathrm{F}} \approx p_{\mathrm{T}}^{\ell \bar{\ell}}$, and therefore the bin limits drive the scale beyond the limit $Q_{\max }^{2}=(1 \mathrm{TeV})^{2}$ defined in section 2.2.1, which we increase for this process to $Q_{\max }^{2}=(10 \mathrm{TeV})^{2}$, along with $N_{\tau}=40$.
} 


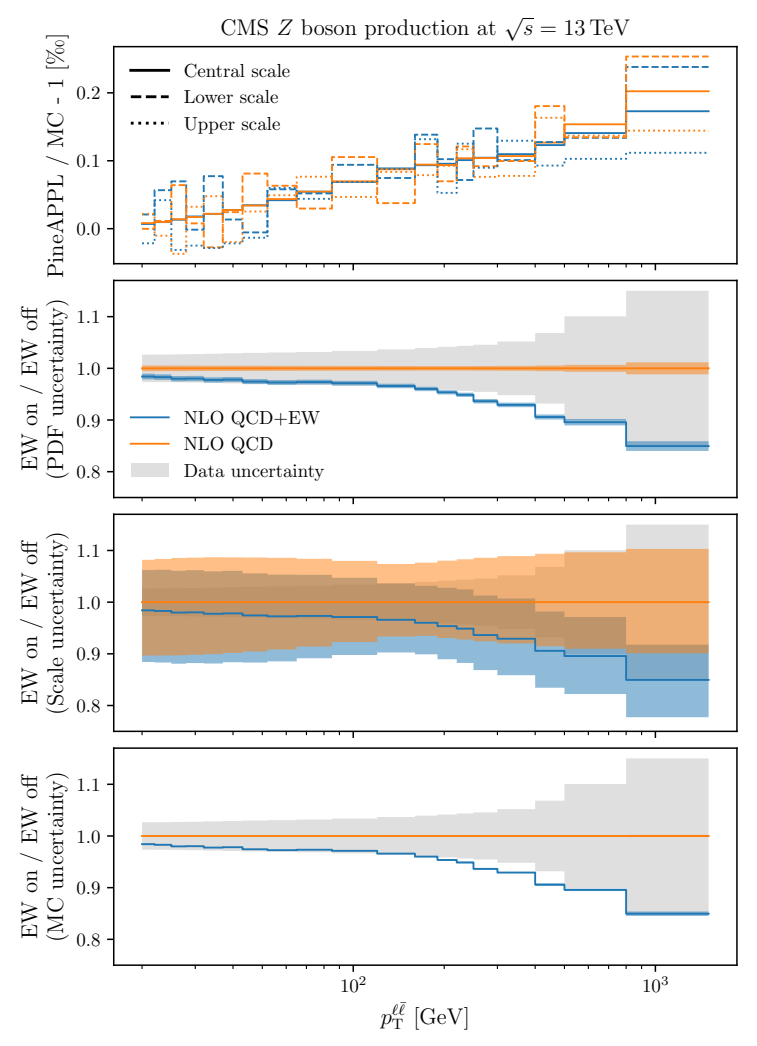

Figure 5. Same as figure 2 but for the CMS differential $\mathrm{Z} p_{\mathrm{T}}$ measurement at a centre-of-mass energy of $13 \mathrm{TeV}$ [112].

in a fit of PDFs to this data set. Finally, the Monte Carlo uncertainty is well under control, as it remains mostly negligible in comparison to the PDF, scale and data uncertainty, and to the size of the EW correction.

\section{Subtraction of EW effects from data}

The ability to perform theoretical calculations simultaneously accurate in both the QCD and EW couplings is not sufficient to make a consistent comparison with experimental measurements. In this section we formulate some guidelines to facilitate this task. We focus on the problem of data with (partially) subtracted EW effects, which, if compared to theory predictions including them, leads to a double counting issue. Our guidelines are intended to make the reader aware of an emerging new issue, whose definitive solution remains however beyond the scope of this work.

A first example is the subtraction of (irreducible) background processes which must not be considered as such. A very blatant case is neutral-current Drell-Yan, where the signal process is the production of an opposite-sign lepton pair, which starts at $\mathcal{O}\left(\alpha^{2}\right)$. Because this process is usually thought as a quark-initiated $s$-channel mechanism $\left(\mathrm{q} \overline{\mathrm{q}} \rightarrow \gamma^{*} / \mathrm{Z} \rightarrow \ell \bar{\ell}\right)$, in many analyses the PI component, $\gamma \gamma \rightarrow \ell \bar{\ell}$ in the $t$ channel, is considered a different process, and therefore as a background and subtracted. The subtraction from the mea- 

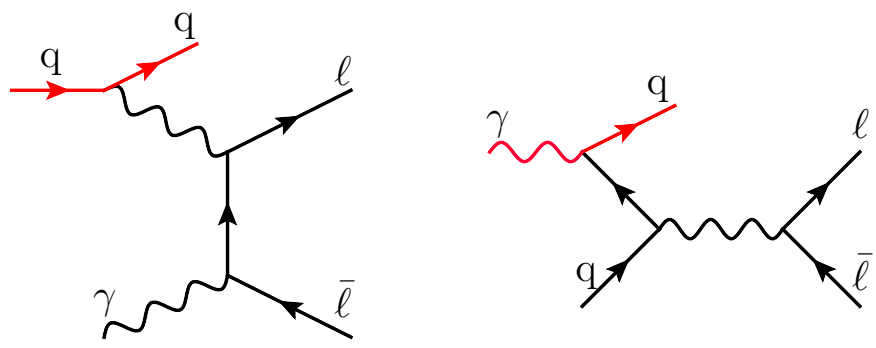

Figure 6. Photon-induced (left) and quark-induced (right) contributions to the Drell-Yan process. In black, the LO process is shown. In red, the initial-state splitting leading to the real-emission $\mathrm{q} \gamma \rightarrow \ell \bar{\ell} \mathrm{q}$ is highlighted. Such a real emission enters in the NLO EW corrections.

sured data is done by calculating the theoretical predictions of the double-photon initiated contribution, possibly including (ill-defined) higher-order corrections. For example, in refs. $[125,126]$ (a similar statement appears also in an older analysis [70]), one reads:

The photon-induced process, $\gamma \gamma \rightarrow \ell \bar{\ell}$, is simulated at LO using Pythia 8 and the MRST2004qed PDF set [16]. The expected yield for this process also accounts for NLO QED/EW corrections from references [127, 128], which decrease the yield by approximately $30 \%$.

Such a distinction, which is unphysical and incorrect in quantum mechanics, may be somehow justified at LO. Beyond this order, it is simply wrong. Indeed, at $\mathcal{O}\left(\alpha^{3}\right)$, the reaction $\mathrm{q} \gamma \rightarrow \ell \bar{\ell} \mathrm{q}$ becomes possible, which includes both kind of topologies discussed above, and needs both in order to yield an IR-finite result, see figure 6 (as a consequence, one cannot speak of EW corrections to $\gamma \gamma \rightarrow \ell \bar{\ell})$. It may be useful for the reader to consider a QCD counterpart of this issue, e.g. the subtraction of the gluon-initiated contribution to top-pair production, in order to understand the incorrectness of this procedure.

A second example is related to removing EW effects from data. These can be either the full EW corrections or just a part of them. In either case, a comparison between these data and a NLO-EW accurate simulation aimed at the extraction of some parameter would be meaningless, as some effects included in the latter have been removed from the former. The typical example relevant for the LHC is the deconvolution of effects due to multiple-photon radiation from light particles in the final state. This applies mostly to processes such as neutral- or charged-current Drell-Yan, especially when electrons are considered. The problem lies in the fact that electrons, and to a lesser extent muons, tend to radiate collinear photons, which are not accounted for in QCD-only matrix elements. Thus, leptons that are measured in the detector are less energetic, and this fact is compensated for by inverting a photon shower. The resulting dataset is e.g. referred to as pre-FSR with observables defined in terms of Born-level electrons (see e.g. ref. [101] for its definition). These datasets are needed for and correctly used in QCD-only PDF determinations, since the EW corrections to some DY observables can be significant, and excluding them would therefore degrade the quality of the fit. In fits including fixed-order EW corrections the problem with this definition, besides double counting, is that the first photon emission is 
included exactly at the matrix-element level. The inclusion of subsequent emission would require the matching with the QED shower, which is not yet available for general processes.

It is interesting to note that one can tune the QED parton shower to mimic NLO EW effects for specific processes and observables, so that a prediction only accurate at NLO QCD displays a remarkable agreement with another at NLO QCD+EW when the photonic shower is included (see also the behaviour of predictions showered with Рнотоs [129-131] in ref. [132]). However, this kind of agreement always comes a posteriori, and cannot be ensured in general. The transverse momentum of the $\mathrm{Z}$ boson as shown in section 3.2.3 is such an example: the difference between the two datasets is rather small (less than $6 \%$ ), but the NLO EW corrections are as large as $25 \%$. Furthermore, the deconvolution of QED effects in data introduces a dependence on the program (and possibly on the specific version) employed for the shower inversion. This fact is especially problematic if deconvolved datasets are the only ones which are published, since undoing the exact deconvolution can be very difficult, or practically impossible.

A more physical definition of leptonic observables would be one making use of either bare leptons (the leptons as they emerge after FSR) or of dressed leptons (leptons and photons are clustered together and their momenta are combined, in analogy with jets in QCD). The problem with the former is that electrons are never measured as bare particles, because of the finite resolution of the electromagnetic calorimeter. For what concerns muons, while in principle the concept of a bare muon is physical, it should be kept in mind that modern, general-purpose codes employed to compute EW corrections treat leptons as massless, to ensure numerical stability of the matrix elements. In this case, using bare leptons is not collinear safe. Dressed leptons avoid all these shortcomings, with the further advantage of being inclusive on the effect of extra collinear emissions. This fact encourages to explore the possibility of employing a dressed-lepton definition, regardless of the leptonic flavour. We acknowledge that this practice is already being followed in (some) experimental analyses: indeed, to mention two examples discussed in this paper, in ref. [101] data for dressed leptons are published, together with the Born-level and bare ones, while refs. [112] employs a dressed-lepton definition. We therefore recommend that these ways of presenting the experimental data become standard in the future.

\section{Conclusions and outlook}

The systematic inclusion of EW corrections in accurate theoretical computations for several LHC processes is becoming more and more important in order to match the increasing precision of the data. In this paper we simplified the computational aspect of this task, building upon the automation of QCD and EW computations pioneered in recent years [9-11]. Specifically we developed PINEAPPL, a new library that stores perturbative calculations from an external Monte Carlo generator in a PDF-independent way using interpolation grids. This offers the advantage of fast a posteriori convolutions with PDFs, for example to study the uncertainties coming from different PDF sets and/or the strong coupling $\alpha_{\mathrm{s}}$, and to determine the PDFs themselves, a task for which fast-interpolation grids are fundamental. We tested PINEAPPL together with MG5_AMC and found a precision of $10^{-4}$ 
to $10^{-5}$ relative to the MC result, which is excellent for all foreseeable practical purposes. Although we used MG5_AMC, we note that PINEAPPL is not tied in any way to a specific Monte Carlo generator, and can be easily interfaced with any of them.

We emphasise that a distinguishing feature of PINEAPPL is the support for arbitrary coupling orders not only in the strong, but also in the electroweak coupling. This enables us to generate, for the first time, NLO EW and NLO combined QCD-EW interpolation grids. Using MG5_AMC we calculated and showcased the impact of these corrections for specific measurements of some representative LHC processes: Drell-Yan lepton-pair production, top-pair production, and Z-boson production with non-zero transverse momentum.

Finally, we discussed the issue of subtracting EW corrections in experimental data, which becomes important when theoretical predictions including EW corrections are compared to experimental data. In particular, with the development of PINEAPPL, all technical requirements are fulfilled for producing the first PDF fit of LHC data including EW and combined QCD-EW corrections. This will have at least two advantages: in PDF fits phase-space regions are usually cut away if they exhibit large EW corrections; including them therefore increases the number of data points in a fit and therefore indirectly enlarges a PDF set's interpolation region. Secondly, this makes it possible to use experimental data that are closer to the actual measurement, without the need to compensate for missing EW corrections. We plan to address this task in a future work.

The PINEAPPL library is available at https://n3pdf.github.io/pineappl.

\section{Acknowledgments}

We would like to thank Valerio Bertone, Stefan Dittmaier, Stefano Forte, Zahari Kassabov, Davide Pagani, and Juan Rojo for a critical reading of the manuscript, and Rikkert Frederix for discussions about AMCFAst. We also acknowledge discussions with Florencia Canelli, Stefano Camarda, Paolo Francavilla, Abideh Jafari, Andreas Jung, Elizaveta Shabalina, Wolfgang Wagner about the treatment of EW effects in experimental data. S.C. and C.S. are supported by the European Research Council under the European Union's Horizon 2020 research and innovation Programme (grant agreement no. 740006). E.R.N. is supported by the European Commission through a Marie Skłodowska-Curie Action (grant agreement no. 752748). M.Z. thanks the Nikhef institute in Amsterdam, where he was employed when this paper was started.

\section{A Installation and usage of PineAPPL}

PINEAPPL currently consists of three parts: 1) the library itself, which is a dependency for the other parts, 2) the helper program pineappl, which allows one to read PINEAPPL grids from the command line and make predictions with it (explained in section A.1), and finally 3) the $\mathrm{C}$ interface, which is intended to be used in Monte Carlo integrators to generate the grids. 


\section{A.1 Demonstration of pineappl}

The program pineappl can be used to perform quick convolutions and other calculations with existing grids on the command line. If started without any arguments, it prints its help and lists all supported subcommands:

$\$$ pineappl

pineappl 0.3 .0

Read, write, and query PineAPPL grids

USAGE :

pineappl <SUBCOMMAND>

FLAGS :

$\begin{array}{ll}\text { - } \mathrm{h}, \text {--help } & \text { Prints help information } \\ -\mathrm{V}, \text {--version } & \text { Prints version information }\end{array}$

SUBCOMMANDS :

$\begin{array}{ll}\text { channels } & \text { Shows the contribution for each partonic channel } \\ \text { convolute } & \text { Convolutes a PineAPPL grid with a PDF set } \\ \text { diff } & \text { Compares the contents of two grids with each other } \\ \text { info } & \text { Shows information about the grid } \\ \text { luminosity } & \text { Shows the luminosity function } \\ \text { merge } & \text { Merges one or more PineAPPL grids together } \\ \text { orders } & \text { Shows the predictions for all bins for each order } \\ & \text { separately } \\ \text { pdf_uncertainty } & \text { Calculates PDF uncertainties }\end{array}$

Convolutions. The most important subcommand is convolute, which performs a convolution of a single grid with a single or multiple PDF sets. As an example we show the grid produced for the ATLAS Drell-Yan high-mass lepton-pair production from section 3.2.1, convoluted with NNPDF31_nlo_as_0118_luxqed as the main PDF set and with CT18NLO as a second PDF set.

\begin{tabular}{|c|c|c|c|c|c|c|c|}
\hline$i^{\prime}$ & xmin & $x \max$ & $\operatorname{diff}$ & integ & neg unc & os unc & CT18NLO \\
\hline 0 & 116 & 130 & $2.0630698 \mathrm{e}-1$ & $2.888297 \mathrm{e} 0$ & $-2.08 \%$ & $1.69 \%$ & $2.0246802 e-1-1.86 \%$ \\
\hline 1 & 130 & 150 & $9.1818985 \mathrm{e}-2$ & $1.836379 \mathrm{e} 0$ & $-1.79 \%$ & $1.86 \%$ & $8.9766355 e-2-2.24 \%$ \\
\hline 2 & 150 & 170 & $4.5306370 \mathrm{e}-2$ & $9.061274 \mathrm{e}-1$ & $-1.60 \%$ & $1.98 \%$ & $4.4115960 \mathrm{e}-2-2.63 \%$ \\
\hline 3 & 170 & 190 & $2.5894856 \mathrm{e}-2$ & $5.178971 e-1$ & $-1.66 \%$ & $2.06 \%$ & $2.5138525 e-2-2.92 \%$ \\
\hline 4 & 190 & 210 & $1.6075267 e-2$ & $3.215053 e-1$ & $-1.70 \%$ & $2.10 \%$ & $1.5566535 e-2-3.16 \%$ \\
\hline 5 & 210 & 230 & $1.0526659 \mathrm{e}-2$ & $2.105331 e-1$ & $-1.71 \%$ & $2.12 \%$ & $1.0173163 e-2-3.36 \%$ \\
\hline 6 & 230 & 250 & $7.1928162 \mathrm{e}-3$ & $1.438563 e-1$ & $-1.71 \%$ & $2.13 \%$ & $6.9403972 e-3-3.51 \%$ \\
\hline
\end{tabular}




$\begin{array}{rrrrrrrrr}7 & 250 & 300 & 4.0776555 \mathrm{e}-3 & 2.038827 \mathrm{e}-1 & -1.70 \% & 2.44 \% & 3.9255068 \mathrm{e}-3 & -3.73 \% \\ 8 & 300 & 400 & 1.4775481 \mathrm{e}-3 & 1.477548 \mathrm{e}-1 & -1.94 \% & 2.87 \% & 1.4182754 \mathrm{e}-3 & -4.01 \% \\ 9 & 400 & 500 & 4.5473785 \mathrm{e}-4 & 4.547378 \mathrm{e}-2 & -2.30 \% & 3.19 \% & 4.3525336 \mathrm{e}-4 & -4.28 \% \\ 10 & 500 & 700 & 1.2164277 \mathrm{e}-4 & 2.432855 \mathrm{e}-2 & -2.41 \% & 3.12 \% & 1.1612523 \mathrm{e}-4 & -4.54 \% \\ 11 & 700 & 1000 & 1.9792340 \mathrm{e}-5 & 5.937701 \mathrm{e}-3 & -2.05 \% & 2.12 \% & 1.8813113 \mathrm{e}-5 & -4.95 \% \\ 12 & 1000 & 1500 & 2.0228761 \mathrm{e}-6 & 1.011438 \mathrm{e}-3 & -1.29 \% & 0.47 \% & 1.9221978 \mathrm{e}-6 & -4.98 \%\end{array}$

The output shows all 13 bins with lower (xmin) and upper limit (xmax) of the invariant mass $M_{\ell \bar{\ell}}$ of the lepton pair, with the differential cross section $\mathrm{d} \sigma / \mathrm{d} M_{\bar{\ell} \bar{\ell}}$ (diff), integrated cross section $\left(M_{\ell \bar{\ell}}^{\max }-M_{\ell \bar{\ell}}^{\min }\right) \mathrm{d} \sigma / \mathrm{d} M_{\ell \bar{\ell}}$ (integ), and the perturbative uncertainty estimated from a 7-point scale variation (envelope given by neg unc and pos unc). The uncertainty estimation can alternatively use a 3 - or a 9-point scale variation using the optional program switch --scales 3 or --scales 9, respectively. The (differential) results for the second PDF set (CT18NLO) is shown in absolute numbers and also as a percentage relative to the result of the first PDF set.

Perturbative orders. Often it is helpful to see the impact of the different perturbative orders to the cross section. The subcommand orders shows this:

\begin{tabular}{|c|c|c|c|c|c|c|}
\hline bin & xmin & $x \max$ & $\operatorname{diff}$ & $0\left(a^{\wedge} 0 a^{\wedge} 2\right)$ & $0\left(a^{\wedge} 1 a^{\wedge} 2\right)$ & $0\left(a^{\wedge} 0 a^{\wedge} 3\right)$ \\
\hline & -1 & - & -- &.- & ---- & -- \\
\hline 0 & 116 & 130 & $2.0630698 \mathrm{e}-1$ & $100.00 \%$ & $15.97 \%$ & $-5.26 \%$ \\
\hline 1 & 130 & 150 & $9.1818985 \mathrm{e}-2$ & $100.00 \%$ & $18.07 \%$ & $-4.29 \%$ \\
\hline 2 & 150 & 170 & $4.5306370 e-2$ & $100.00 \%$ & $19.66 \%$ & $-3.64 \%$ \\
\hline 3 & 170 & 190 & $2.5894856 \mathrm{e}-2$ & $100.00 \%$ & $20.69 \%$ & $-3.21 \%$ \\
\hline 4 & 190 & 210 & $1.6075267 e-2$ & $100.00 \%$ & $21.26 \%$ & $-2.91 \%$ \\
\hline 5 & 210 & 230 & $1.0526659 \mathrm{e}-2$ & $100.00 \%$ & $21.48 \%$ & $-2.85 \%$ \\
\hline 6 & 230 & 250 & $7.1928162 \mathrm{e}-3$ & $100.00 \%$ & $21.60 \%$ & $-2.62 \%$ \\
\hline 7 & 250 & 300 & $4.0776555 \mathrm{e}-3$ & $100.00 \%$ & $21.36 \%$ & $-2.75 \%$ \\
\hline 8 & 300 & 400 & $1.4775481 \mathrm{e}-3$ & $100.00 \%$ & $20.32 \%$ & $-3.11 \%$ \\
\hline 9 & 400 & 500 & $4.5473785 e-4$ & $100.00 \%$ & $17.83 \%$ & $-3.65 \%$ \\
\hline 10 & 500 & 700 & $1.2164277 \mathrm{e}-4$ & $100.00 \%$ & $14.04 \%$ & $-4.68 \%$ \\
\hline 11 & 700 & 1000 & $1.9792340 e-5$ & $100.00 \%$ & $7.21 \%$ & $-6.75 \%$ \\
\hline 12 & 1000 & 1500 & $2.0228761 e-6$ & $100.00 \%$ & $-3.05 \%$ & $-9.99 \%$ \\
\hline
\end{tabular}

The first four columns are the same as in convolute, and the remaining ones show all orders normalised to the sum of the leading orders, which in this case is only the $\mathcal{O}\left(\alpha^{2}\right)$. Absolute numbers are shown if the switch --absolute or -a is passed to the program.

Channels and luminosity function. Sometimes it is useful to know which partons contribute the most and by how much. This is what the subcommand channels shows: 


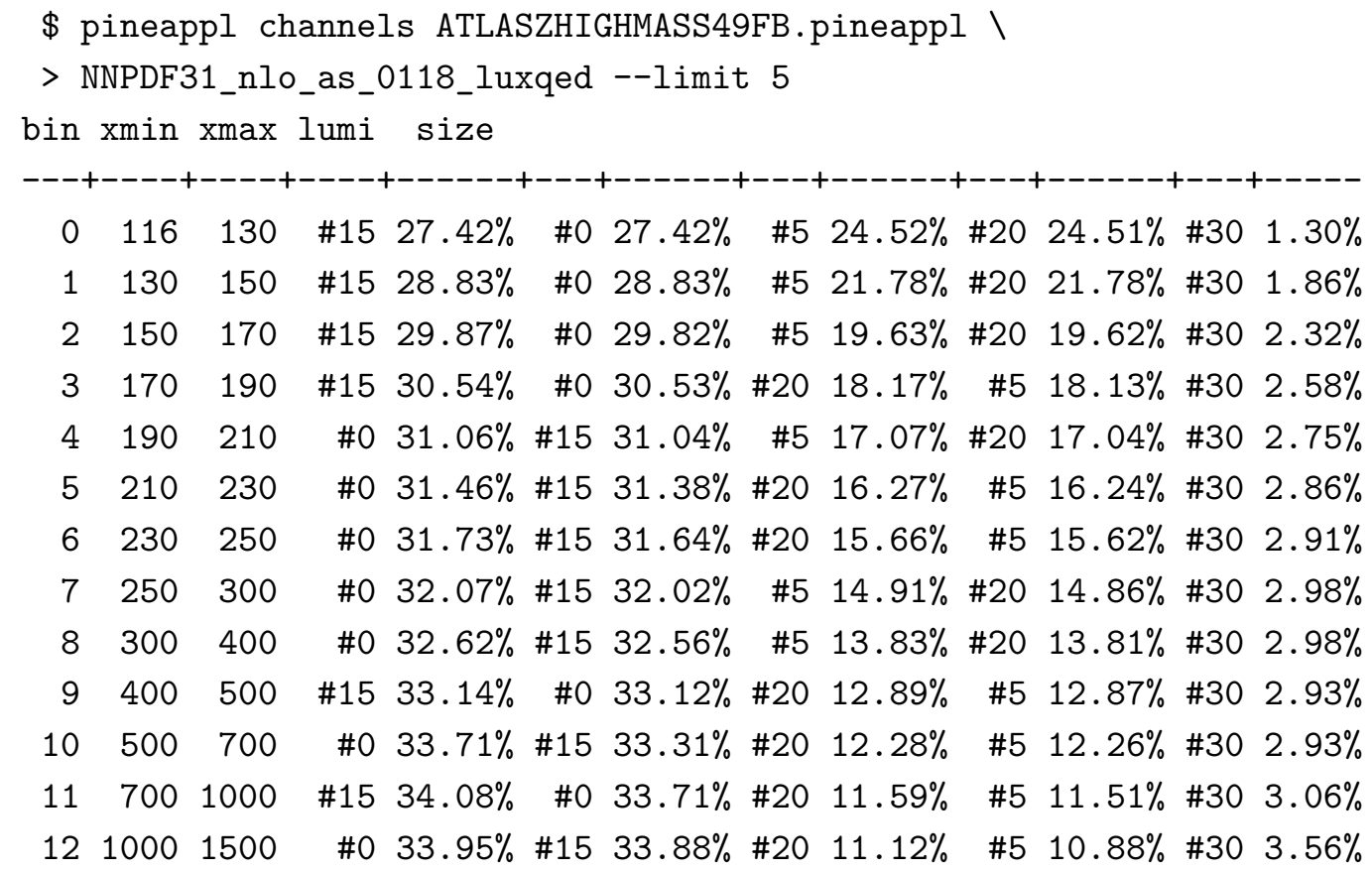

The first three columns are known from convolute. The next columns (the switch --limit 5 limits the output to five columns) show first the channel index and then the relative size of the corresponding contribution. Since the contribution of a partonic channel can be negative, the columns are sorted ignoring the sign of the contribution. The first line shows that for bin 0 , i.e. for the range $116 \mathrm{GeV}<M_{\ell \bar{\ell}}<130 \mathrm{GeV}$, the cross section is dominated by partonic channel \#15 (27.42\%), following by partonic channel \#0 with same size, then channel \#5, etc. The meaning of the channel numbers is given by using the subcommand luminosity (only an excerpt is shown):

id entry

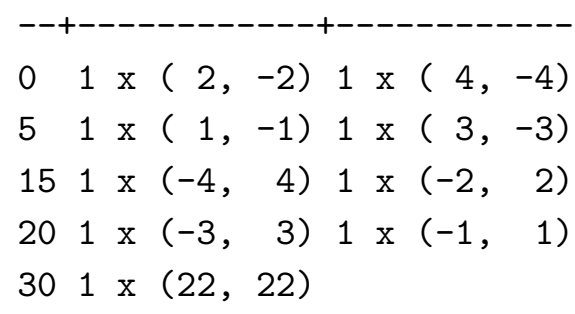

This shows that channel \#0 represents the up-type quark-anti-quark contributions (shown with PDG id 2 and 4 for up and charm quarks, which have the same matrix elements), channel \#15 is the same channel with its initial states transposed, channels \#5 and \#20 are the down-type quark-anti-quark channels, and channel \#30 is the photon-photon channel. The size of the remaining channels is smaller than the photon-photon channel. The factors 1 are not important here, but in general they can contain CKM values and charge factors that, if kept in the squared matrix elements, would not allow for sharing a single matrix element for different quark flavours and therefore slow down the calculation. A complete list of all channels and of their contribution to the cross section for all of the processes discussed in section 3 is collected in appendix B. 


\section{A.2 Sample runcard for mg5_aMC@NLO}

The following run card was used to produce the results shown in section 3.2.1. The only difference with respect to a standard MG5_AMC run is the switch set pineappl True, which enables to fill a PINEAPPL grid. For a complete set of runcards and patches see https://n3pdf.github.io/pineappl.

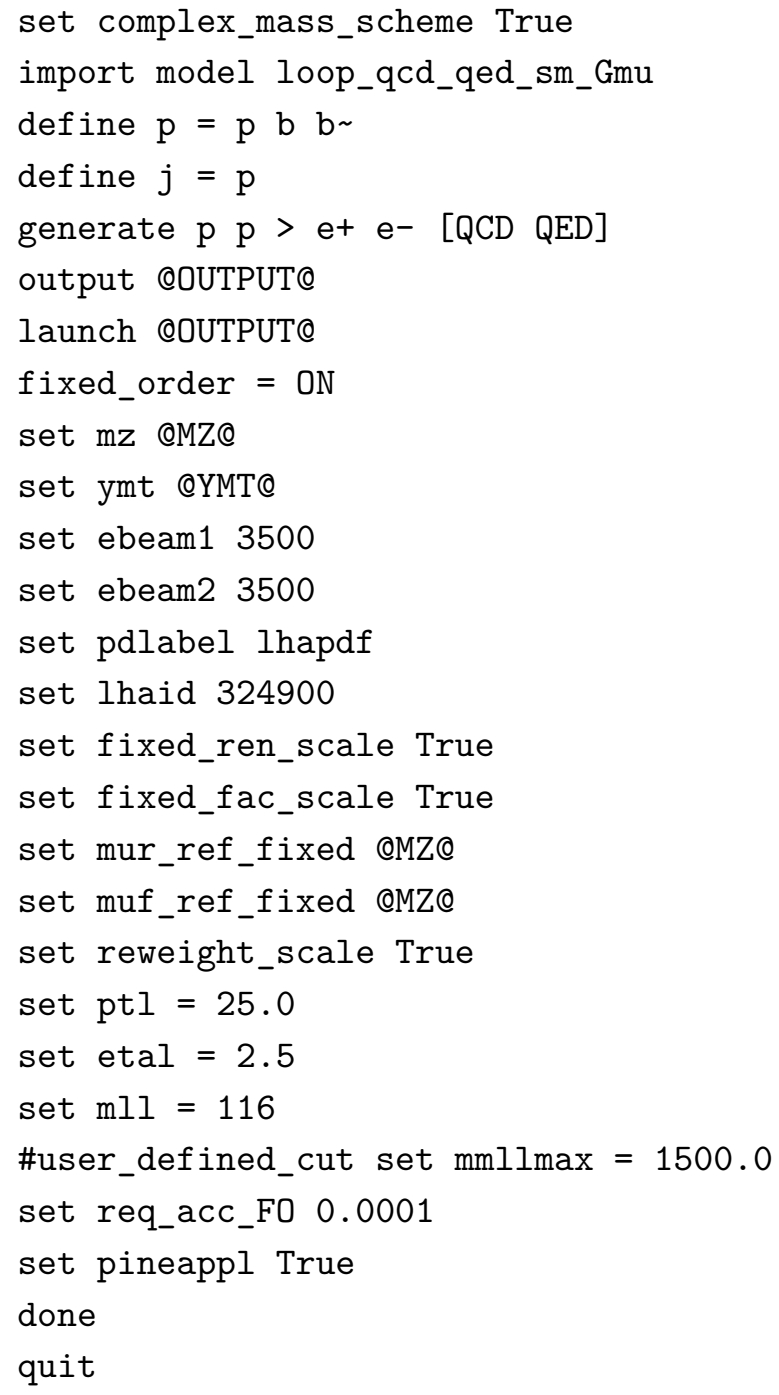

\section{A.3 Example Monte Carlo program in $\mathrm{C}++$}

The following listing shows how to setup PINEAPPL using its C interface in a simple Monte Carlo integrator for calculating the double-photon contribution to Drell-Yan lepton-pair production at the LHC. All PINEAPPL functions have the prefix pineappl_. The full example together with a makefile can be found at https://github.com/N3PDF/pineappl/tree/ master/examples/capi-dy-aa. The documentation of the $\mathrm{C}$ API can be found at https://docs.rs/pineappl_capi. 


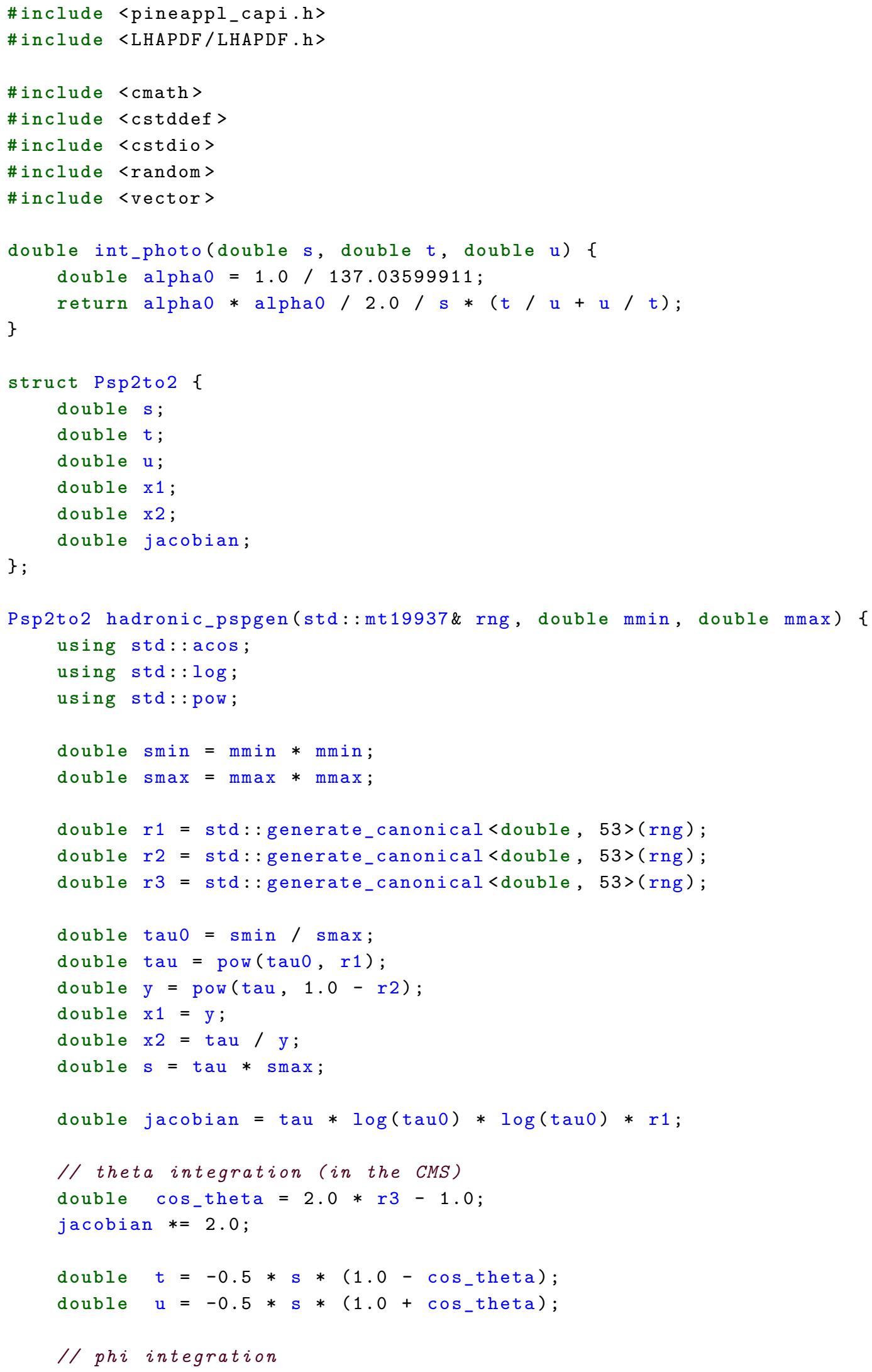




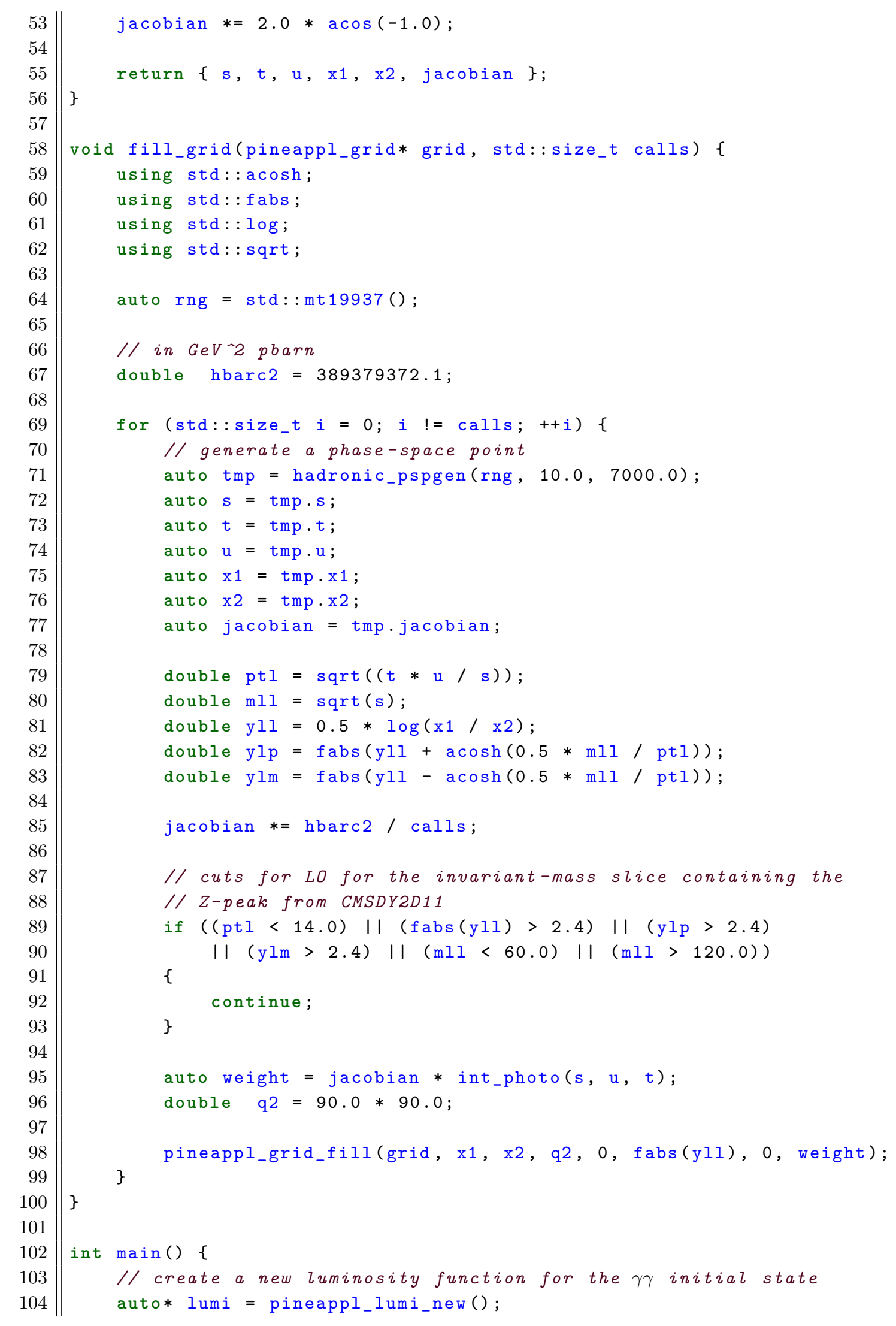




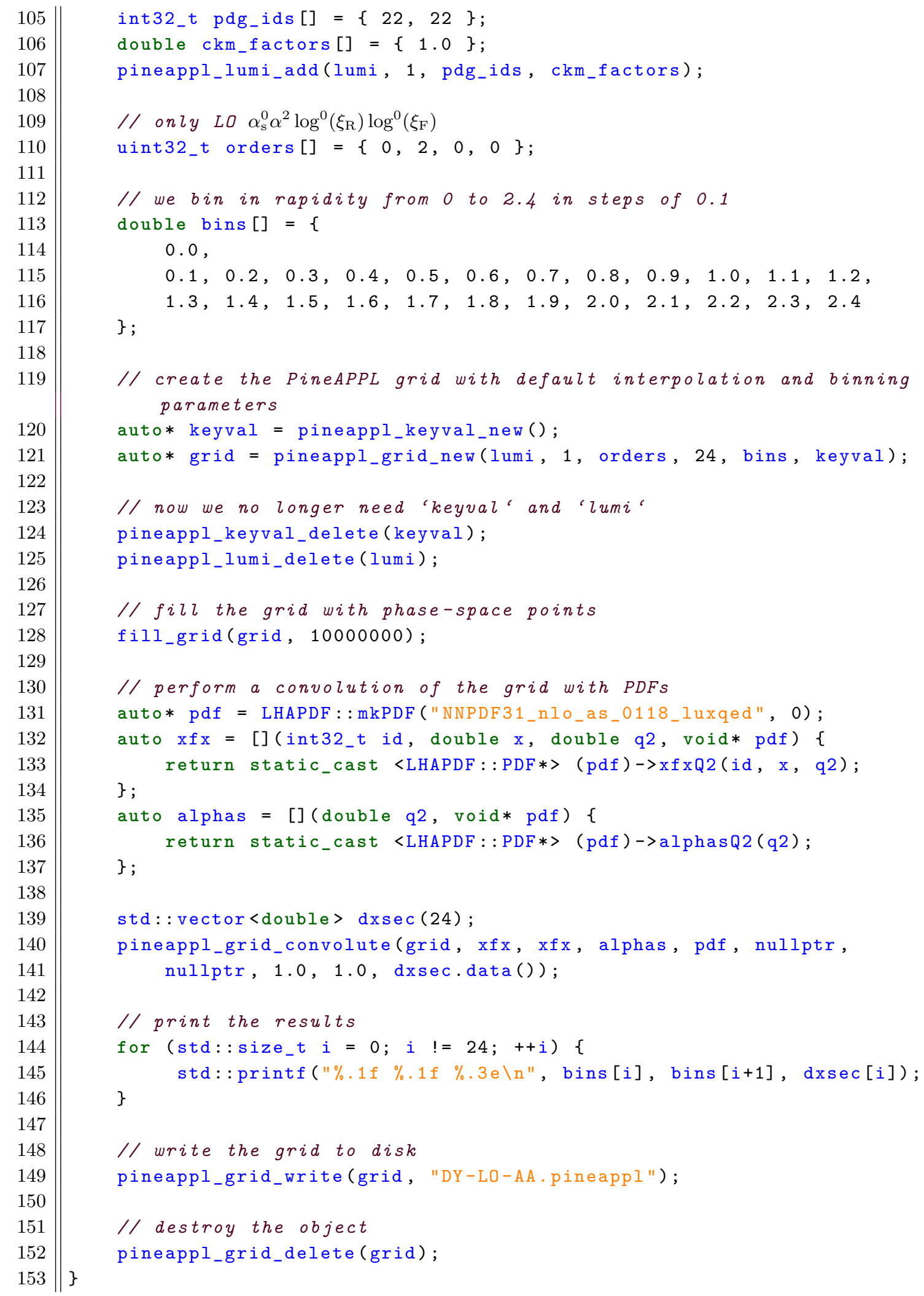




\section{A.4 Installation}

Updated installation instructions are kept in the file README. md in PINEAPPL's repository at https://github.com/N3PDF/pineappl/ and on its homepage https://n3pdf.github.io/ pineappl/.

\section{Installation of Rust}

All parts are written in Rust: a Rust compiler and related tools are needed. On operating systems with a bash shell (such as Linux or MacOS) the installation is as simple as

$$
\text { \$ curl --proto '=https' --tlsv1.2 -sSf https://sh.rustup.rs I sh }
$$

which downloads the compiler rustc, the package manager cargo, and a few other helpful tools. When the installation has completed make sure to read and follow the instructions printed on screen. See also https://www.rust-lang.org/tools/install for more details and for installation instructions for other operating systems.

\section{Installation of the command-line program pineappl}

The command-line program pineappl is compiled and installed using

\$ cargo install pineappl_cli

This program also needs LHAPDF [133] installed; make sure that the environment variables PATH, LD_LIBRARY_PATH, and PKG_CONFIG_PATH are properly set. For usage instructions simply type pineappl in your shell and read the help message.

\section{Installation of the C-language interface (optional)}

For the $\mathrm{C}$ interface you need to first install cargo-c,

\$ cargo install cargo-c

and then download the PINEAPPL repository, compile and finally install into it into a directory \$prefix as follows:

\$ git clone https://github.com/N3PDF/pineappl/

\$ cd pineappl_capi/

$\$$ cargo cinstall --release --prefix=DIRECTORY

The last line will install the $\mathrm{C}$ header pineappl_capi.h, the library, and a pkg-config ${ }^{10}$ file (pineappl_capi.pc) into the directory specified as DIRECTORY. Make sure that the environment variables PATH, LD_LIBRARY_PATH, and PKG_CONFIG_PATH are properly set. The latter is needed for pkg-config --cflags --libs pineappl_capi to work, which prints the necessary compiler/linker flags.

After being installed, one can compile and link against the library. See appendix A.3 for an example. 


\begin{tabular}{|c|c|c|c|c|c|c|c|c|c|c|c|c|c|c|c|c|}
\hline & & & & 1 & 2 & 3 & 4 & 5 & 6 & 7 & 8 & 9 & 10 & 11 & 12 & 13 \\
\hline 1 & $u, \bar{u}$ & $c, \bar{c}$ & & 27.42 & 28.83 & 29.82 & 30.53 & 31.06 & 31.46 & 31.73 & 32.07 & 32.62 & 33.12 & 33.71 & 33.71 & 33.95 \\
\hline 2 & $\gamma, \bar{c}$ & $\gamma, \bar{u}$ & & -0.03 & -0.03 & -0.03 & -0.03 & -0.02 & -0.01 & -0.01 & 0.00 & 0.01 & 0.01 & 0.02 & 0.02 & 0.02 \\
\hline 3 & $g, \bar{c}$ & $g, \bar{u}$ & & -0.77 & -0.58 & -0.39 & -0.27 & -0.17 & -0.10 & -0.05 & 0.02 & 0.08 & 0.12 & 0.13 & 0.10 & 0.09 \\
\hline 4 & $u, \gamma$ & $c, \gamma$ & & -0.09 & -0.10 & -0.09 & -0.07 & -0.05 & -0.02 & 0.01 & 0.05 & 0.11 & 0.19 & 0.21 & 0.30 & 0.40 \\
\hline 5 & $u, g$ & $c, g$ & & -0.76 & -0.42 & -0.08 & 0.19 & 0.42 & 0.59 & 0.76 & 0.93 & 1.22 & 1.50 & 1.68 & 1.88 & 2.15 \\
\hline 6 & $d, \bar{d}$ & $s, \bar{s}$ & & 24.52 & 21.78 & 19.63 & 18.13 & 17.07 & 16.24 & 15.62 & 14.91 & 13.83 & 12.87 & 12.26 & 11.51 & 10.88 \\
\hline 7 & $\gamma, \bar{s}$ & $\gamma, \bar{d}$ & & 0.00 & 0.00 & 0.00 & 0.00 & 0.00 & 0.00 & 0.00 & 0.00 & 0.00 & 0.00 & 0.00 & 0.00 & 0.00 \\
\hline 8 & $g, \bar{s}$ & $g, \bar{d}$ & & -0.84 & -0.55 & -0.34 & -0.21 & -0.13 & -0.06 & -0.02 & 0.02 & 0.08 & 0.10 & 0.10 & 0.09 & 0.07 \\
\hline 9 & $d, \gamma$ & $s, \gamma$ & & 0.00 & 0.00 & 0.00 & 0.00 & 0.01 & 0.01 & 0.01 & 0.01 & 0.01 & 0.01 & 0.01 & 0.01 & 0.01 \\
\hline 10 & $d, g$ & $s, g$ & & -0.89 & -0.53 & -0.27 & -0.10 & 0.01 & 0.10 & 0.17 & 0.23 & 0.33 & 0.39 & 0.42 & 0.45 & 0.44 \\
\hline 11 & $b, \bar{b}$ & & & 1.15 & 0.92 & 0.74 & 0.60 & 0.51 & 0.43 & 0.39 & 0.31 & 0.22 & 0.14 & 0.09 & 0.05 & 0.02 \\
\hline 12 & $\gamma, \bar{b}$ & & & 0.00 & 0.00 & 0.00 & 0.00 & 0.00 & 0.00 & 0.00 & 0.00 & 0.00 & 0.00 & 0.00 & 0.00 & 0.00 \\
\hline 13 & $g, \bar{b}$ & & & -0.17 & -0.11 & -0.07 & -0.05 & -0.03 & -0.02 & -0.01 & 0.00 & 0.00 & 0.01 & 0.01 & 0.01 & 0.01 \\
\hline 14 & $b, \gamma$ & & & 0.00 & 0.00 & 0.00 & 0.00 & 0.00 & 0.00 & 0.00 & 0.00 & 0.00 & 0.00 & 0.00 & 0.00 & 0.00 \\
\hline 15 & $b, g$ & & & -0.17 & -0.11 & -0.07 & -0.04 & -0.03 & -0.02 & -0.01 & 0.00 & 0.00 & 0.01 & 0.01 & 0.01 & 0.00 \\
\hline 16 & $\bar{c}, c$ & $\bar{u}, u$ & & 27.42 & 28.83 & 29.87 & 30.54 & 31.04 & 31.38 & 31.64 & 32.02 & 32.56 & 33.14 & 33.31 & 34.08 & 33.88 \\
\hline 17 & $\gamma, u$ & $\gamma, c$ & & -0.09 & -0.10 & -0.09 & -0.07 & -0.04 & -0.03 & -0.01 & 0.04 & 0.11 & 0.18 & 0.23 & 0.31 & 0.44 \\
\hline 18 & $g, u$ & $g, c$ & & -0.77 & -0.42 & -0.08 & 0.19 & 0.41 & 0.59 & 0.73 & 0.93 & 1.23 & 1.48 & 1.68 & 1.90 & 2.12 \\
\hline 19 & $\bar{c}, \gamma$ & $\bar{u}, \gamma$ & & -0.03 & -0.03 & -0.03 & -0.03 & -0.02 & -0.01 & -0.01 & 0.00 & 0.01 & 0.01 & 0.02 & 0.02 & 0.02 \\
\hline 20 & $\bar{c}, g$ & $\bar{u}, g$ & & -0.76 & -0.57 & -0.40 & -0.27 & -0.17 & -0.10 & -0.04 & 0.02 & 0.08 & 0.12 & 0.13 & 0.11 & 0.09 \\
\hline 21 & $\bar{s}, s$ & $\bar{d}, d$ & & 24.51 & 21.78 & 19.62 & 18.17 & 17.04 & 16.27 & 15.66 & 14.86 & 13.81 & 12.89 & 12.28 & 11.59 & 11.12 \\
\hline 22 & $\gamma, d$ & $\gamma, s$ & & 0.00 & 0.00 & 0.00 & 0.01 & 0.01 & 0.01 & 0.01 & 0.01 & 0.01 & 0.01 & 0.01 & 0.01 & 0.01 \\
\hline 23 & $g, d$ & $g, s$ & & -0.88 & -0.53 & -0.26 & -0.10 & 0.01 & 0.10 & 0.15 & 0.24 & 0.32 & 0.39 & 0.43 & 0.44 & 0.43 \\
\hline 24 & $\bar{s}, \gamma$ & $\bar{d}, \gamma$ & & 0.00 & 0.00 & 0.00 & 0.00 & 0.00 & 0.00 & 0.00 & 0.00 & 0.00 & 0.00 & 0.00 & 0.00 & 0.00 \\
\hline 25 & $\bar{s}, g$ & $\bar{d}, g$ & & -0.84 & -0.55 & -0.34 & -0.21 & -0.12 & -0.06 & -0.02 & 0.03 & 0.07 & 0.10 & 0.10 & 0.09 & 0.07 \\
\hline 26 & $\bar{b}, b$ & & & 1.15 & 0.92 & 0.73 & 0.60 & 0.51 & 0.44 & 0.38 & 0.31 & 0.22 & 0.14 & 0.09 & 0.05 & 0.02 \\
\hline 27 & $\gamma, b$ & & & 0.00 & 0.00 & 0.00 & 0.00 & 0.00 & 0.00 & 0.00 & 0.00 & 0.00 & 0.00 & 0.00 & 0.00 & 0.00 \\
\hline 28 & $\underline{g}, b$ & & & -0.17 & -0.11 & -0.07 & -0.04 & -0.03 & -0.02 & -0.01 & 0.00 & 0.00 & 0.01 & 0.01 & 0.01 & 0.01 \\
\hline 29 & $\bar{b}, \gamma$ & & & 0.00 & 0.00 & 0.00 & 0.00 & 0.00 & 0.00 & 0.00 & 0.00 & 0.00 & 0.00 & 0.00 & 0.00 & 0.00 \\
\hline 30 & $\bar{b}, g$ & & & -0.17 & -0.11 & -0.07 & -0.04 & -0.03 & -0.02 & -0.01 & 0.00 & 0.00 & 0.01 & 0.01 & 0.01 & 0.01 \\
\hline 31 & $\underline{\gamma}, \gamma$ & & & 1.30 & 1.86 & 2.32 & 2.58 & 2.75 & 2.86 & 2.91 & 2.98 & 2.98 & 2.93 & 2.93 & 3.06 & 3.56 \\
\hline 32 & $\bar{b}, \gamma$ & $\bar{s}, \gamma$ & $\bar{d}, \gamma$ & -0.01 & -0.01 & -0.01 & -0.01 & 0.00 & 0.00 & 0.00 & 0.00 & 0.01 & 0.01 & 0.01 & 0.01 & 0.01 \\
\hline 33 & $d, \underline{\gamma}$ & $s, \gamma$ & $b, \gamma$ & -0.02 & -0.02 & -0.02 & -0.01 & -0.01 & 0.00 & 0.01 & 0.01 & 0.03 & 0.04 & 0.05 & 0.07 & 0.08 \\
\hline 34 & $\gamma, \bar{b}$ & $\gamma, \bar{s}$ & $\gamma, \bar{d}$ & -0.01 & -0.01 & -0.01 & -0.01 & -0.01 & 0.00 & 0.00 & 0.00 & 0.01 & 0.01 & 0.01 & 0.02 & 0.01 \\
\hline 35 & $\gamma, d$ & $\gamma, s$ & $\gamma, b$ & -0.02 & -0.02 & -0.02 & -0.01 & -0.01 & 0.00 & 0.01 & 0.02 & 0.03 & 0.03 & 0.05 & 0.07 & 0.08 \\
\hline
\end{tabular}

Table 1. The 35 parton luminosities contributing to the predictions, accurate to NLO QCD+EW, of the Drell-Yan lepton-pair production measured by the ATLAS experiment at a centre-of-mass energy of $7 \mathrm{TeV}$ [70]. The contributions are reported, in percentage, for each of the 13 bins. The bin edges are in the invariant mass of the lepton pair, $M_{\ell \bar{\ell}}$, as follows: 116, 130, 150, 170, 190, 210, 230, 250, 300, 400, 500, 700, 1000, $1500 \mathrm{GeV}$.

\section{B Parton luminosities}

In this appendix, we present the complete breakdown of parton luminosities entering the $\mathrm{NLO} \mathrm{QCD}+\mathrm{EW}$ predictions of the various measurements considered in section 3. For each parton luminosity, we indicate its percentage contribution to the cross section in a given bin. In particular:

- Table 1 collects the 35 parton luminosities contributing to the Drell-Yan leptonpair production measured by the ATLAS experiment at a centre-of-mass energy of $7 \mathrm{TeV}[70]$. The 13 bins are for the invariant mass of the lepton pair, $M_{\ell \bar{\ell}}$, with edges at $116,130,150,170,190,210,230,250,300,400,500,700,1000$ and $1500 \mathrm{GeV}$.

- Tables 2-7 collect the 35 parton luminosities contributing to the double differential Drell-Yan lepton-pair production measured by the CMS experiment at a centre-ofmass energy of $7 \mathrm{TeV}$ [70]. The 132 bins are split across tables $2-7$, where each table corresponds to a bin in the invariant mass of the lepton pair: table 2 to $20 \mathrm{GeV}<$ $M_{\ell \bar{\ell}}<30 \mathrm{GeV}$; table 3 to $30 \mathrm{GeV}<M_{\ell \bar{\ell}}<45 \mathrm{GeV}$; table 4 to $45 \mathrm{GeV}<M_{\ell \bar{\ell}}<60 \mathrm{GeV}$;

\footnotetext{
${ }^{10} \mathrm{~A}$ standard way on Linux to express how dependencies are compiled/linked against, see https://www.freedesktop.org/wiki/Software/pkg-config/.
} 


\begin{tabular}{|c|c|c|c|c|c|c|c|c|c|c|c|c|c|c|c|}
\hline & & & & 1 & 2 & 3 & 4 & 5 & 6 & 7 & 8 & 9 & 10 & 11 & 12 \\
\hline 1 & $u, \bar{u}$ & $c, \bar{c}$ & & 16.87 & 17.00 & 16.94 & 17.06 & 16.99 & 17.02 & 17.03 & 17.19 & 17.50 & 17.21 & 17.34 & 17.44 \\
\hline 2 & $\gamma, \bar{c}$ & $\gamma, \bar{u}$ & & 0.22 & 0.23 & 0.23 & 0.22 & 0.23 & 0.23 & 0.22 & 0.24 & 0.23 & 0.23 & 0.22 & 0.23 \\
\hline 3 & $g, \bar{c}$ & $g, \bar{u}$ & & 8.98 & 8.93 & 8.96 & 8.84 & 8.91 & 8.76 & 8.76 & 8.63 & 8.51 & 8.38 & 8.44 & 8.20 \\
\hline 4 & $u, \gamma$ & $c, \gamma$ & & 0.56 & 0.56 & 0.56 & 0.57 & 0.54 & 0.55 & 0.58 & 0.56 & 0.55 & 0.54 & 0.52 & 0.55 \\
\hline 5 & $u, g$ & $c, g$ & & 10.87 & 10.88 & 10.89 & 10.89 & 10.96 & 10.86 & 11.14 & 11.06 & 10.94 & 11.31 & 11.31 & 11.44 \\
\hline 6 & $d, \bar{d}$ & $s, \bar{s}$ & & 5.12 & 5.21 & 5.15 & 5.12 & 5.23 & 5.11 & 5.17 & 5.13 & 5.11 & 5.15 & 5.07 & 5.11 \\
\hline 7 & $\gamma, \bar{s}$ & $\gamma, \bar{d}$ & & 0.00 & 0.01 & 0.01 & 0.00 & 0.00 & 0.01 & 0.01 & 0.00 & 0.00 & 0.00 & 0.01 & 0.00 \\
\hline 8 & $g, \bar{s}$ & $g, \bar{d}$ & & 2.72 & 2.69 & 2.71 & 2.66 & 2.71 & 2.63 & 2.64 & 2.64 & 2.59 & 2.57 & 2.58 & 2.53 \\
\hline 9 & $d, \gamma$ & $s, \gamma$ & & 0.01 & 0.00 & 0.01 & 0.01 & 0.00 & 0.00 & 0.01 & 0.00 & 0.01 & 0.01 & 0.01 & 0.01 \\
\hline 10 & $d, g$ & $s, g$ & & 2.91 & 2.96 & 2.94 & 3.00 & 2.97 & 2.94 & 2.92 & 2.92 & 2.98 & 2.93 & 2.93 & 2.95 \\
\hline 11 & $b, \bar{b}$ & & & 0.29 & 0.32 & 0.32 & 0.30 & 0.29 & 0.30 & 0.30 & 0.30 & 0.30 & 0.30 & 0.28 & 0.28 \\
\hline 12 & $\gamma, \bar{b}$ & & & 0.00 & 0.00 & 0.00 & 0.00 & 0.00 & 0.00 & 0.00 & 0.00 & 0.00 & 0.00 & 0.00 & 0.00 \\
\hline 13 & $g, \bar{b}$ & & & 0.48 & 0.45 & 0.44 & 0.47 & 0.45 & 0.46 & 0.44 & 0.44 & 0.43 & 0.43 & 0.43 & 0.42 \\
\hline 14 & $b, \gamma$ & & & 0.00 & 0.00 & 0.00 & 0.00 & 0.00 & 0.00 & 0.00 & 0.00 & 0.00 & 0.00 & 0.00 & 0.00 \\
\hline 15 & $b, g$ & & & 0.47 & 0.48 & 0.44 & 0.48 & 0.48 & 0.45 & 0.47 & 0.44 & 0.42 & 0.43 & 0.43 & 0.40 \\
\hline 16 & $\bar{c}, c$ & $\bar{u}, u$ & & 17.00 & 16.92 & 16.99 & 17.05 & 17.01 & 17.18 & 16.99 & 17.20 & 17.16 & 17.27 & 17.49 & 17.38 \\
\hline 17 & $\gamma, u$ & $\gamma, c$ & & 0.58 & 0.57 & 0.57 & 0.57 & 0.54 & 0.56 & 0.54 & 0.55 & 0.57 & 0.55 & 0.56 & 0.58 \\
\hline 18 & $g, u$ & $g, c$ & & 10.82 & 10.74 & 10.86 & 10.74 & 10.81 & 10.92 & 11.07 & 10.96 & 11.23 & 11.33 & 11.28 & 11.55 \\
\hline 19 & $\bar{c}, \gamma$ & $\bar{u}, \gamma$ & & 0.22 & 0.22 & 0.23 & 0.22 & 0.23 & 0.23 & 0.22 & 0.23 & 0.22 & 0.22 & 0.24 & 0.24 \\
\hline 20 & $\bar{c}, g$ & $\bar{u}, g$ & & 8.96 & 8.95 & 8.92 & 8.93 & 8.87 & 8.88 & 8.69 & 8.69 & 8.59 & 8.42 & 8.34 & 8.27 \\
\hline 21 & $\bar{s}, s$ & $\bar{d}, d$ & & 5.12 & 5.10 & 5.06 & 5.11 & 5.12 & 5.18 & 5.10 & 5.19 & 5.14 & 5.11 & 5.12 & 5.09 \\
\hline 22 & $\gamma, d$ & $\gamma, s$ & & 0.00 & 0.01 & 0.00 & 0.01 & 0.00 & 0.01 & 0.00 & 0.01 & 0.00 & 0.00 & 0.01 & 0.00 \\
\hline 23 & $g, d$ & $g, s$ & & 2.94 & 2.93 & 2.95 & 2.98 & 2.92 & 2.92 & 2.94 & 2.91 & 2.92 & 2.97 & 2.91 & 2.93 \\
\hline 24 & $\bar{s}, \gamma$ & $\bar{d}, \gamma$ & & 0.01 & 0.01 & 0.00 & 0.01 & 0.00 & 0.00 & 0.01 & 0.00 & 0.01 & 0.01 & 0.01 & 0.01 \\
\hline 25 & $\bar{s}, g$ & $\bar{d}, g$ & & 2.73 & 2.72 & 2.71 & 2.71 & 2.67 & 2.69 & 2.70 & 2.63 & 2.58 & 2.62 & 2.52 & 2.49 \\
\hline 26 & $\bar{b}, b$ & & & 0.29 & 0.31 & 0.31 & 0.29 & 0.29 & 0.31 & 0.28 & 0.30 & 0.28 & 0.29 & 0.28 & 0.26 \\
\hline 27 & $\gamma, b$ & & & 0.00 & 0.00 & 0.00 & 0.00 & 0.00 & 0.00 & 0.00 & 0.00 & 0.00 & 0.00 & 0.00 & 0.00 \\
\hline 28 & $g, b$ & & & 0.46 & 0.44 & 0.45 & 0.44 & 0.48 & 0.46 & 0.47 & 0.44 & 0.45 & 0.45 & 0.40 & 0.39 \\
\hline 29 & $\bar{b}, \gamma$ & & & 0.00 & 0.00 & 0.00 & 0.00 & 0.00 & 0.00 & 0.00 & 0.00 & 0.00 & 0.00 & 0.00 & 0.00 \\
\hline 30 & $\bar{b}, g$ & & & 0.46 & 0.48 & 0.47 & 0.45 & 0.42 & 0.45 & 0.43 & 0.44 & 0.42 & 0.44 & 0.42 & 0.42 \\
\hline 31 & $\gamma, \gamma$ & & & 0.49 & 0.48 & 0.48 & 0.48 & 0.46 & 0.49 & 0.49 & 0.48 & 0.45 & 0.46 & 0.45 & 0.44 \\
\hline 32 & $\bar{b}, \gamma$ & $\bar{s}, \gamma$ & $\bar{d}, \gamma$ & 0.08 & 0.07 & 0.08 & 0.08 & 0.07 & 0.08 & 0.08 & 0.08 & 0.08 & 0.07 & 0.08 & 0.07 \\
\hline 33 & $d, \gamma$ & $s, \gamma$ & $b, \gamma$ & 0.13 & 0.12 & 0.12 & 0.11 & 0.12 & 0.12 & 0.12 & 0.12 & 0.12 & 0.12 & 0.12 & 0.13 \\
\hline 34 & $\gamma, \bar{b}$ & $\gamma, \bar{s}$ & $\gamma, \bar{d}$ & 0.07 & 0.08 & 0.07 & 0.07 & 0.08 & 0.08 & 0.08 & 0.07 & 0.08 & 0.07 & 0.08 & 0.08 \\
\hline \multirow{2}{*}{35} & $\gamma, d$ & $\gamma, s$ & $\gamma, b$ & 0.12 & 0.12 & 0.12 & 0.12 & 0.11 & 0.11 & 0.12 & 0.12 & 0.11 & 0.11 & 0.12 & 0.12 \\
\hline & & & & 13 & 14 & 15 & 16 & 17 & 18 & 19 & 20 & 21 & 22 & 23 & 24 \\
\hline 1 & $u, \bar{u}$ & $c, \bar{c}$ & & 17.52 & 17.69 & 17.89 & 17.93 & 18.41 & 18.50 & 19.19 & 19.83 & 20.68 & 21.20 & 22.55 & 22.96 \\
\hline 2 & $\gamma, \bar{c}$ & $\gamma, \bar{u}$ & & 0.25 & 0.24 & 0.22 & 0.24 & 0.21 & 0.23 & 0.20 & 0.23 & 0.21 & 0.17 & 0.15 & 0.15 \\
\hline 3 & $g, \bar{c}$ & $g, \bar{u}$ & & 8.07 & 8.02 & 7.66 & 7.55 & 7.26 & 7.08 & 6.61 & 6.25 & 5.81 & 5.60 & 4.91 & 4.89 \\
\hline 4 & $u, \gamma$ & $c, \gamma$ & & 0.55 & 0.56 & 0.54 & 0.53 & 0.49 & 0.50 & 0.49 & 0.41 & 0.39 & 0.40 & 0.39 & 0.59 \\
\hline 5 & $u, g$ & $c, g$ & & 11.56 & 11.75 & 11.67 & 11.91 & 11.96 & 12.01 & 12.01 & 11.97 & 11.80 & 11.68 & 11.28 & 12.60 \\
\hline 6 & $d, \bar{d}$ & $s, \bar{s}$ & & 5.09 & 5.05 & 5.06 & 5.11 & 5.16 & 5.03 & 5.18 & 5.34 & 5.45 & 5.41 & 5.47 & 5.61 \\
\hline 7 & $\gamma, \bar{s}$ & $\gamma, \bar{d}$ & & 0.01 & 0.00 & 0.00 & 0.00 & 0.01 & 0.00 & 0.01 & 0.00 & 0.00 & 0.00 & 0.00 & 0.00 \\
\hline 8 & $g, \bar{s}$ & $g, \bar{d}$ & & 2.44 & 2.45 & 2.41 & 2.33 & 2.28 & 2.16 & 2.13 & 2.02 & 1.86 & 1.82 & 1.63 & 1.41 \\
\hline 9 & $d, \gamma$ & $s, \gamma$ & & 0.01 & 0.00 & 0.01 & 0.00 & 0.00 & 0.00 & 0.00 & 0.01 & 0.00 & 0.01 & 0.01 & 0.00 \\
\hline 10 & $d, g$ & $s, g$ & & 2.91 & 2.94 & 2.93 & 2.87 & 2.82 & 2.87 & 2.81 & 2.76 & 2.66 & 2.49 & 2.45 & 2.09 \\
\hline 11 & $b, \bar{b}$ & & & 0.27 & 0.26 & 0.25 & 0.25 & 0.23 & 0.25 & 0.24 & 0.26 & 0.20 & 0.26 & 0.19 & 0.16 \\
\hline 12 & $\gamma, \bar{b}$ & & & 0.00 & 0.00 & 0.00 & 0.00 & 0.00 & 0.00 & 0.00 & 0.00 & 0.00 & 0.00 & 0.00 & 0.01 \\
\hline 13 & $g, \bar{b}$ & & & 0.41 & 0.37 & 0.39 & 0.36 & 0.36 & 0.32 & 0.31 & 0.28 & 0.30 & 0.27 & 0.16 & 0.22 \\
\hline 14 & $b, \gamma$ & & & 0.00 & 0.00 & 0.00 & 0.00 & 0.00 & 0.00 & 0.00 & 0.00 & 0.00 & 0.00 & 0.00 & 0.00 \\
\hline 15 & $b, g$ & & & 0.40 & 0.38 & 0.36 & 0.36 & 0.34 & 0.33 & 0.29 & 0.28 & 0.27 & 0.27 & 0.20 & 0.23 \\
\hline 16 & $\bar{c}, c$ & $\bar{u}, u$ & & 17.71 & 17.50 & 18.22 & 18.23 & 18.33 & 18.76 & 19.13 & 19.71 & 20.69 & 21.12 & 21.51 & 21.39 \\
\hline 17 & $\gamma, u$ & $\gamma, c$ & & 0.54 & 0.55 & 0.56 & 0.53 & 0.54 & 0.51 & 0.49 & 0.44 & 0.55 & 0.41 & 0.25 & 0.43 \\
\hline 18 & $g, u$ & $g, c$ & & 11.47 & 11.74 & 11.73 & 11.98 & 12.01 & 12.13 & 12.16 & 11.89 & 11.61 & 11.72 & 11.91 & 12.47 \\
\hline 19 & $\bar{c}, \gamma$ & $\bar{u}, \gamma$ & & 0.23 & 0.22 & 0.23 & 0.23 & 0.22 & 0.23 & 0.20 & 0.20 & 0.21 & 0.17 & 0.13 & 0.19 \\
\hline 20 & $\bar{c}, g$ & $\bar{u}, g$ & & 8.05 & 7.97 & 7.64 & 7.48 & 7.32 & 7.11 & 6.79 & 6.25 & 5.81 & 5.44 & 5.06 & 4.10 \\
\hline 21 & $\bar{s}, s$ & $\bar{d}, d$ & & 5.16 & 5.08 & 5.07 & 5.07 & 5.07 & 5.13 & 5.15 & 5.31 & 5.35 & 5.75 & 5.94 & 5.34 \\
\hline 22 & $\gamma, d$ & $\gamma, s$ & & 0.01 & 0.00 & 0.00 & 0.01 & 0.01 & 0.01 & 0.00 & 0.00 & 0.00 & 0.00 & 0.00 & 0.01 \\
\hline 23 & $g, d$ & $g, s$ & & 2.90 & 2.94 & 2.93 & 2.86 & 2.87 & 2.84 & 2.78 & 2.80 & 2.67 & 2.56 & 2.31 & 2.29 \\
\hline 24 & $\bar{s}, \gamma$ & $\bar{d}, \gamma$ & & 0.00 & 0.00 & 0.00 & 0.00 & 0.01 & 0.00 & 0.00 & 0.00 & 0.00 & 0.01 & 0.00 & 0.01 \\
\hline 25 & $\bar{s}, g$ & $\bar{d}, g$ & & 2.51 & 2.42 & 2.38 & 2.34 & 2.24 & 2.23 & 2.10 & 2.15 & 1.87 & 1.69 & 1.86 & 1.58 \\
\hline 26 & $\bar{b}, b$ & & & 0.27 & 0.26 & 0.27 & 0.24 & 0.24 & 0.24 & 0.23 & 0.21 & 0.21 & 0.21 & 0.24 & 0.21 \\
\hline 27 & $\gamma, b$ & & & 0.00 & 0.00 & 0.00 & 0.00 & 0.00 & 0.00 & 0.00 & 0.00 & 0.00 & 0.00 & 0.00 & 0.00 \\
\hline 28 & $g, b$ & & & 0.43 & 0.37 & 0.38 & 0.38 & 0.34 & 0.34 & 0.34 & 0.27 & 0.27 & 0.26 & 0.30 & 0.22 \\
\hline 29 & $\bar{b}, \gamma$ & & & 0.00 & 0.00 & 0.00 & 0.00 & 0.00 & 0.00 & 0.00 & 0.00 & 0.00 & 0.00 & 0.00 & 0.00 \\
\hline 30 & $\bar{b}, g$ & & & 0.40 & 0.38 & 0.38 & 0.37 & 0.34 & 0.33 & 0.30 & 0.31 & 0.27 & 0.24 & 0.16 & 0.19 \\
\hline 31 & $\gamma, \gamma$ & & & 0.46 & 0.46 & 0.44 & 0.46 & 0.56 & 0.47 & 0.50 & 0.52 & 0.55 & 0.55 & 0.59 & 0.50 \\
\hline 32 & $\bar{b}, \gamma$ & $\bar{s}, \gamma$ & $\bar{d}, \gamma$ & 0.08 & 0.08 & 0.08 & 0.08 & 0.07 & 0.07 & 0.07 & 0.07 & 0.05 & 0.06 & 0.10 & 0.00 \\
\hline 33 & $d, \gamma$ & $s, \gamma$ & $b, \gamma$ & 0.12 & 0.12 & 0.11 & 0.11 & 0.10 & 0.10 & 0.11 & 0.10 & 0.09 & 0.11 & 0.06 & 0.05 \\
\hline 34 & $\gamma, \bar{b}$ & $\gamma, \bar{s}$ & $\gamma, \bar{d}$ & 0.08 & 0.08 & 0.09 & 0.07 & 0.08 & 0.08 & 0.07 & 0.07 & 0.06 & 0.05 & 0.06 & 0.06 \\
\hline 35 & $\gamma, d$ & $\gamma, s$ & $\gamma, b$ & 0.10 & 0.12 & 0.10 & 0.11 & 0.12 & 0.10 & 0.11 & 0.07 & 0.09 & 0.07 & 0.09 & 0.04 \\
\hline
\end{tabular}

Table 2. The 35 parton luminosities contributing to the predictions, accurate to NLO QCD+EW, of the double differential Drell-Yan lepton pair production measured by the CMS experiment at a centre-of-mass energy of $7 \mathrm{TeV}$ [71]. The invariant mass bin is $20 \mathrm{GeV}<M_{\ell \bar{\ell}}<30 \mathrm{GeV}$; bins 1-24 are in the rapidity of the lepton pair, $0.0<y_{\ell \bar{\ell}}<2.4$, and each bin has a width of 0.1. 


\begin{tabular}{|c|c|c|c|c|c|c|c|c|c|c|c|c|c|c|c|}
\hline & & & & 25 & 26 & 27 & 28 & 29 & 30 & 31 & 32 & 33 & 34 & 35 & 36 \\
\hline 1 & $u, \bar{u}$ & $c, \bar{c}$ & & 35.63 & 35.65 & 35.58 & 35.60 & 35.68 & 35.68 & 35.77 & 35.85 & 35.81 & 36.05 & 35.97 & 36.15 \\
\hline 2 & $\gamma, \bar{c}$ & $\gamma, \bar{u}$ & & -0.01 & -0.02 & -0.02 & -0.02 & -0.02 & -0.02 & -0.01 & -0.02 & -0.01 & -0.02 & -0.01 & -0.02 \\
\hline 3 & $g, \bar{c}$ & $g, \bar{u}$ & & 0.03 & 0.03 & 0.07 & 0.05 & 0.03 & 0.02 & -0.03 & -0.05 & -0.03 & -0.07 & -0.10 & -0.15 \\
\hline 4 & $u, \gamma$ & $c, \gamma$ & & 0.03 & 0.02 & 0.03 & 0.04 & 0.04 & 0.03 & 0.02 & 0.01 & 0.02 & 0.01 & 0.01 & 0.01 \\
\hline 5 & $u, g$ & $c, g$ & & 0.51 & 0.50 & 0.54 & 0.53 & 0.56 & 0.60 & 0.60 & 0.61 & 0.66 & 0.66 & 0.67 & 0.69 \\
\hline 6 & $d, \bar{d}$ & $s, \bar{s}$ & & 11.37 & 11.33 & 11.40 & 11.33 & 11.33 & 11.32 & 11.29 & 11.25 & 11.22 & 11.15 & 11.09 & 11.11 \\
\hline 7 & $\gamma, \bar{s}$ & $\gamma, \bar{d}$ & & 0.01 & 0.01 & 0.01 & 0.01 & 0.01 & 0.01 & 0.01 & 0.01 & 0.01 & 0.01 & 0.01 & 0.01 \\
\hline 8 & $g, \bar{s}$ & $g, \bar{d}$ & & 0.04 & 0.04 & 0.03 & 0.05 & 0.03 & 0.01 & 0.04 & 0.01 & 0.01 & -0.01 & 0.00 & -0.02 \\
\hline 9 & $d, \gamma$ & $s, \gamma$ & & 0.01 & 0.01 & 0.01 & 0.01 & 0.01 & 0.01 & 0.01 & 0.01 & 0.01 & 0.01 & 0.01 & 0.01 \\
\hline 10 & $d, g$ & $s, g$ & & 0.10 & 0.09 & 0.11 & 0.10 & 0.10 & 0.08 & 0.11 & 0.11 & 0.13 & 0.08 & 0.10 & 0.09 \\
\hline 11 & $b, \bar{b}$ & & & 0.86 & 0.84 & 0.86 & 0.84 & 0.85 & 0.84 & 0.83 & 0.80 & 0.81 & 0.78 & 0.77 & 0.74 \\
\hline 12 & $\gamma, \bar{b}$ & & & 0.00 & 0.00 & 0.00 & 0.00 & 0.00 & 0.00 & 0.00 & 0.00 & 0.00 & 0.00 & 0.00 & 0.00 \\
\hline 13 & $g, \bar{b}$ & & & -0.02 & -0.01 & -0.02 & -0.02 & -0.01 & -0.03 & -0.02 & -0.01 & -0.01 & -0.03 & -0.02 & -0.01 \\
\hline 14 & $b, \gamma$ & & & 0.00 & 0.00 & 0.00 & 0.00 & 0.00 & 0.00 & 0.00 & 0.00 & 0.00 & 0.00 & 0.00 & 0.00 \\
\hline 15 & $b, g$ & & & -0.03 & -0.03 & -0.01 & -0.02 & -0.01 & -0.02 & -0.03 & -0.01 & -0.02 & -0.03 & -0.01 & -0.03 \\
\hline 16 & $\bar{c}, c$ & $\bar{u}, u$ & & 35.61 & 35.82 & 35.58 & 35.73 & 35.66 & 35.79 & 35.70 & 35.82 & 35.96 & 36.04 & 36.06 & 36.24 \\
\hline 17 & $\gamma, u$ & $\gamma, c$ & & 0.03 & 0.02 & 0.02 & 0.03 & 0.03 & 0.04 & 0.03 & 0.02 & 0.02 & 0.01 & 0.01 & 0.01 \\
\hline 18 & $g, u$ & $g, c$ & & 0.51 & 0.49 & 0.58 & 0.57 & 0.59 & 0.56 & 0.62 & 0.58 & 0.63 & 0.67 & 0.69 & 0.70 \\
\hline 19 & $\bar{c}, \gamma$ & $\bar{u}, \gamma$ & & -0.02 & -0.02 & -0.01 & -0.01 & -0.01 & -0.02 & -0.01 & -0.02 & -0.02 & -0.02 & -0.03 & -0.01 \\
\hline 20 & $\bar{c}, g$ & $\bar{u}, g$ & & 0.05 & -0.01 & 0.03 & 0.04 & 0.03 & 0.00 & 0.02 & -0.04 & -0.05 & -0.07 & -0.09 & -0.11 \\
\hline 21 & $\bar{s}, s$ & $\bar{d}, d$ & & 11.37 & 11.34 & 11.35 & 11.32 & 11.25 & 11.32 & 11.22 & 11.26 & 11.17 & 11.13 & 11.17 & 11.04 \\
\hline 22 & $\gamma, d$ & $\gamma, s$ & & 0.01 & 0.01 & 0.01 & 0.01 & 0.01 & 0.01 & 0.01 & 0.01 & 0.01 & 0.01 & 0.01 & 0.01 \\
\hline 23 & $g, d$ & $g, s$ & & 0.10 & 0.10 & 0.09 & 0.09 & 0.11 & 0.12 & 0.11 & 0.09 & 0.09 & 0.10 & 0.15 & 0.09 \\
\hline 24 & $\bar{s}, \gamma$ & $\bar{d}, \gamma$ & & 0.01 & 0.01 & 0.01 & 0.01 & 0.01 & 0.01 & 0.01 & 0.01 & 0.01 & 0.01 & 0.01 & 0.01 \\
\hline 25 & $\bar{s}, g$ & $\bar{d}, g$ & & 0.04 & 0.03 & 0.05 & 0.04 & 0.03 & 0.02 & 0.03 & 0.01 & -0.01 & -0.01 & -0.01 & -0.03 \\
\hline 26 & $\bar{b}, b$ & & & 0.86 & 0.86 & 0.86 & 0.84 & 0.85 & 0.82 & 0.84 & 0.83 & 0.79 & 0.80 & 0.78 & 0.75 \\
\hline 27 & $\gamma, b$ & & & 0.00 & 0.00 & 0.00 & 0.00 & 0.00 & 0.00 & 0.00 & 0.00 & 0.00 & 0.00 & 0.00 & 0.00 \\
\hline 28 & $g, b$ & & & -0.01 & -0.01 & -0.02 & -0.03 & -0.02 & -0.02 & -0.02 & -0.02 & -0.01 & -0.04 & -0.02 & -0.03 \\
\hline 29 & $\bar{b}, \gamma$ & & & 0.00 & 0.00 & 0.00 & 0.00 & 0.00 & 0.00 & 0.00 & 0.00 & 0.00 & 0.00 & 0.00 & 0.00 \\
\hline 30 & $\bar{b}, g$ & & & -0.01 & -0.02 & -0.02 & -0.03 & -0.01 & -0.01 & -0.02 & -0.01 & -0.02 & -0.02 & -0.03 & -0.02 \\
\hline 31 & $\underline{\gamma}, \gamma$ & & & 2.97 & 2.93 & 2.94 & 2.94 & 2.93 & 2.89 & 2.93 & 2.91 & 2.88 & 2.85 & 2.85 & 2.83 \\
\hline 32 & $\bar{b}, \gamma$ & $\bar{s}, \gamma$ & $\bar{d}, \gamma$ & -0.01 & -0.01 & -0.01 & -0.02 & -0.01 & -0.01 & -0.01 & -0.01 & -0.01 & -0.01 & -0.01 & -0.01 \\
\hline 33 & $d, \underline{\gamma}$ & $s, \gamma$ & $b, \gamma$ & -0.01 & 0.00 & 0.00 & -0.01 & 0.00 & -0.01 & 0.00 & -0.01 & -0.01 & -0.01 & -0.01 & -0.01 \\
\hline 34 & $\gamma, \bar{b}$ & $\gamma, \bar{s}$ & $\gamma, \bar{d}$ & -0.01 & -0.01 & -0.01 & -0.01 & -0.01 & -0.01 & -0.01 & -0.01 & -0.01 & -0.02 & -0.01 & -0.01 \\
\hline \multirow[t]{2}{*}{35} & $\gamma, d$ & $\gamma, s$ & $\gamma, b$ & -0.01 & -0.01 & -0.01 & -0.01 & -0.01 & 0.00 & -0.01 & -0.01 & -0.01 & -0.01 & -0.01 & -0.01 \\
\hline & & & & 37 & 38 & 39 & 40 & 41 & 42 & 43 & 44 & 45 & 46 & 47 & 48 \\
\hline 1 & $u, \bar{u}$ & $c, \bar{c}$ & & 36.47 & 36.82 & 37.42 & 37.93 & 38.69 & 39.38 & 40.18 & 40.67 & 41.34 & 40.60 & 41.28 & 40.83 \\
\hline 2 & $\gamma, \bar{c}$ & $\gamma, \bar{u}$ & & -0.02 & -0.04 & -0.05 & -0.06 & -0.08 & -0.07 & -0.08 & -0.10 & -0.10 & -0.10 & -0.12 & 0.09 \\
\hline 3 & $g, \bar{c}$ & $g, \bar{u}$ & & -0.21 & -0.33 & -0.55 & -0.74 & -0.98 & -1.16 & -1.44 & -1.63 & -1.78 & -1.51 & -1.24 & -1.86 \\
\hline 4 & $u, \gamma$ & $c, \gamma$ & & 0.01 & -0.03 & -0.05 & -0.08 & -0.11 & -0.16 & -0.14 & -0.17 & -0.20 & -0.17 & -0.27 & -0.07 \\
\hline 5 & $u, g$ & $c, g$ & & 0.61 & 0.48 & 0.33 & 0.09 & -0.12 & -0.41 & -0.73 & -0.96 & -0.94 & -0.74 & -0.57 & -0.03 \\
\hline 6 & $d, \bar{d}$ & $s, \bar{s}$ & & 11.08 & 11.12 & 11.06 & 11.20 & 11.18 & 11.27 & 11.34 & 11.29 & 11.21 & 11.07 & 10.90 & 10.72 \\
\hline 7 & $\gamma, \bar{s}$ & $\gamma, \bar{d}$ & & 0.01 & 0.00 & 0.01 & 0.00 & 0.00 & 0.00 & 0.00 & 0.00 & 0.00 & 0.00 & 0.00 & 0.00 \\
\hline 8 & $g, \bar{s}$ & $g, \bar{d}$ & & -0.05 & -0.11 & -0.13 & -0.22 & -0.27 & -0.34 & -0.42 & -0.48 & -0.51 & -0.42 & -0.44 & -0.59 \\
\hline 9 & $d, \gamma$ & $s, \gamma$ & & 0.01 & 0.01 & 0.01 & 0.01 & 0.01 & 0.01 & 0.01 & 0.01 & 0.00 & 0.01 & 0.00 & 0.00 \\
\hline 10 & $d, g$ & $s, g$ & & 0.09 & 0.03 & -0.05 & -0.09 & -0.16 & -0.24 & -0.35 & -0.36 & -0.38 & -0.33 & -0.27 & -0.02 \\
\hline 11 & $b, \bar{b}$ & & & 0.73 & 0.71 & 0.70 & 0.70 & 0.72 & 0.64 & 0.62 & 0.64 & 0.63 & 0.53 & 0.52 & 0.48 \\
\hline 12 & $\gamma, \bar{b}$ & & & 0.00 & 0.00 & 0.00 & 0.00 & 0.00 & 0.00 & 0.00 & 0.00 & 0.00 & 0.00 & 0.00 & 0.01 \\
\hline 13 & $g, \bar{b}$ & & & -0.03 & -0.04 & -0.05 & -0.06 & -0.08 & -0.07 & -0.10 & -0.08 & -0.11 & -0.07 & -0.06 & -0.10 \\
\hline 14 & $b, \gamma$ & & & 0.00 & 0.00 & 0.00 & 0.00 & 0.00 & 0.00 & 0.00 & 0.00 & 0.00 & 0.00 & 0.00 & 0.00 \\
\hline 15 & $b, g$ & & & -0.02 & -0.04 & -0.05 & -0.05 & -0.06 & -0.08 & -0.10 & -0.11 & -0.11 & -0.10 & -0.12 & -0.10 \\
\hline 16 & $\bar{c}, c$ & $\bar{u}, u$ & & 36.33 & 36.88 & 37.44 & 37.92 & 38.62 & 39.18 & 39.94 & 40.88 & 40.88 & 41.01 & 40.33 & 40.59 \\
\hline 17 & $\gamma, u$ & $\gamma, c$ & & -0.02 & -0.04 & -0.05 & -0.07 & -0.10 & -0.15 & -0.17 & -0.19 & -0.16 & -0.15 & -0.16 & -0.12 \\
\hline 18 & $g, u$ & $g, c$ & & 0.64 & 0.54 & 0.36 & 0.12 & -0.12 & -0.43 & -0.70 & -1.05 & -0.94 & -0.67 & -0.53 & -0.31 \\
\hline 19 & $\bar{c}, \gamma$ & $\bar{u}, \gamma$ & & -0.02 & -0.03 & -0.04 & -0.07 & -0.08 & -0.08 & -0.08 & -0.11 & -0.10 & -0.11 & -0.09 & -0.12 \\
\hline 20 & $\bar{c}, g$ & $\bar{u}, g$ & & -0.21 & -0.35 & -0.54 & -0.72 & -0.98 & -1.18 & -1.45 & -1.70 & -1.66 & -1.48 & -1.50 & -1.62 \\
\hline 21 & $\bar{s}, s$ & $\bar{d}, d$ & & 11.11 & 11.07 & 11.07 & 11.21 & 11.24 & 11.36 & 11.38 & 11.42 & 11.02 & 10.85 & 10.86 & 10.41 \\
\hline 22 & $\gamma, d$ & $\gamma, s$ & & 0.01 & 0.00 & 0.01 & 0.01 & 0.01 & 0.01 & 0.01 & 0.01 & 0.00 & 0.00 & 0.01 & 0.00 \\
\hline 23 & $g, d$ & $g, s$ & & 0.06 & 0.05 & 0.00 & -0.12 & -0.13 & -0.24 & -0.34 & -0.38 & -0.40 & -0.37 & -0.41 & -0.12 \\
\hline 24 & $\bar{s}, \gamma$ & $\bar{d}, \gamma$ & & 0.01 & 0.00 & 0.01 & 0.01 & 0.00 & 0.00 & 0.01 & 0.00 & 0.00 & 0.00 & 0.00 & 0.00 \\
\hline 25 & $\bar{s}, g$ & $\bar{d}, g$ & & -0.03 & -0.10 & -0.17 & -0.21 & -0.30 & -0.35 & -0.45 & -0.49 & -0.49 & -0.50 & -0.45 & -0.45 \\
\hline 26 & $\bar{b}, b$ & & & 0.74 & 0.71 & 0.70 & 0.70 & 0.67 & 0.67 & 0.65 & 0.64 & 0.62 & 0.53 & 0.51 & 0.53 \\
\hline 27 & $\gamma, b$ & & & 0.00 & 0.00 & 0.00 & 0.00 & 0.00 & 0.00 & 0.00 & 0.00 & 0.00 & 0.00 & 0.00 & 0.00 \\
\hline 28 & $\underline{g}, b$ & & & -0.03 & -0.04 & -0.05 & -0.06 & -0.06 & -0.08 & -0.09 & -0.10 & -0.09 & -0.09 & -0.12 & -0.03 \\
\hline 29 & $\bar{b}, \gamma$ & & & 0.00 & 0.00 & 0.00 & 0.00 & 0.00 & 0.00 & 0.00 & 0.00 & 0.00 & 0.00 & 0.00 & 0.00 \\
\hline 30 & $\bar{b}, g$ & & & -0.03 & -0.03 & -0.05 & -0.06 & -0.06 & -0.08 & -0.08 & -0.11 & -0.11 & -0.09 & -0.14 & -0.12 \\
\hline 31 & $\underline{\gamma}, \gamma$ & & & 2.81 & 2.82 & 2.83 & 2.80 & 2.68 & 2.76 & 2.74 & 2.65 & 2.52 & 2.44 & 2.26 & 2.13 \\
\hline 32 & $\bar{b}, \gamma$ & $\bar{s}, \gamma$ & $\bar{d}, \gamma$ & -0.01 & -0.02 & -0.02 & -0.02 & -0.03 & -0.04 & -0.03 & -0.04 & -0.04 & -0.03 & -0.07 & -0.03 \\
\hline 33 & $d, \underline{\gamma}$ & $s, \gamma$ & $b, \gamma$ & -0.01 & -0.02 & -0.02 & -0.03 & -0.04 & -0.04 & -0.05 & -0.05 & -0.05 & -0.05 & -0.04 & -0.09 \\
\hline 34 & $\gamma, \bar{b}$ & $\gamma, \bar{s}$ & $\gamma, \bar{d}$ & -0.01 & -0.02 & -0.02 & -0.02 & -0.03 & -0.04 & -0.04 & -0.05 & -0.04 & -0.04 & -0.03 & -0.02 \\
\hline 35 & $\gamma, d$ & $\gamma, s$ & $\gamma, b$ & -0.01 & -0.02 & -0.02 & -0.02 & -0.04 & -0.04 & -0.04 & -0.06 & -0.05 & -0.04 & -0.05 & -0.01 \\
\hline
\end{tabular}

Table 3. Same as table 2, but for the invariant mass bin $30 \mathrm{GeV}<M_{\ell \bar{\ell}}<45 \mathrm{GeV}$. 


\begin{tabular}{|c|c|c|c|c|c|c|c|c|c|c|c|c|c|c|c|}
\hline & & & & 49 & 50 & 51 & 52 & 53 & 54 & 55 & 56 & 57 & 58 & 59 & 60 \\
\hline 1 & $u, \bar{u}$ & $c, \bar{c}$ & & 35.55 & 35.51 & 35.54 & 35.66 & 35.51 & 35.53 & 35.70 & 35.67 & 36.27 & 36.28 & 36.60 & 37.17 \\
\hline 2 & $\gamma, \bar{c}$ & $\gamma, \bar{u}$ & & -0.08 & -0.08 & -0.07 & -0.09 & -0.08 & -0.07 & -0.09 & -0.09 & -0.08 & -0.10 & -0.10 & -0.13 \\
\hline 3 & $g, \bar{c}$ & $g, \bar{u}$ & & -1.29 & -1.29 & -1.34 & -1.35 & -1.30 & -1.32 & -1.32 & -1.35 & -1.40 & -1.43 & -1.56 & -1.67 \\
\hline 4 & $u, \gamma$ & $c, \gamma$ & & -0.13 & -0.13 & -0.11 & -0.09 & -0.14 & -0.10 & -0.13 & -0.13 & -0.16 & -0.16 & -0.22 & -0.25 \\
\hline 5 & $u, g$ & $c, g$ & & -1.10 & -1.09 & -1.08 & -1.07 & -1.08 & -1.04 & -1.11 & -1.03 & -1.13 & -1.15 & -1.31 & -1.47 \\
\hline 6 & $d, \bar{d}$ & $s, \bar{s}$ & & 15.51 & 15.31 & 15.25 & 15.23 & 15.35 & 15.22 & 15.13 & 15.08 & 15.03 & 14.85 & 15.15 & 15.04 \\
\hline 7 & $\gamma, \bar{s}$ & $\gamma, \bar{d}$ & & 0.01 & 0.01 & 0.01 & 0.01 & 0.01 & 0.01 & 0.01 & 0.00 & 0.01 & 0.01 & 0.00 & 0.01 \\
\hline 8 & $g, \bar{s}$ & $g, \bar{d}$ & & -0.48 & -0.46 & -0.43 & -0.48 & -0.45 & -0.49 & -0.47 & -0.53 & -0.50 & -0.56 & -0.57 & -0.62 \\
\hline 9 & $d, \gamma$ & $s, \gamma$ & & 0.01 & 0.01 & 0.01 & 0.01 & 0.01 & 0.01 & 0.01 & 0.01 & 0.01 & 0.01 & 0.01 & 0.01 \\
\hline 10 & $d, g$ & $s, g$ & & -0.48 & -0.42 & -0.45 & -0.43 & -0.44 & -0.46 & -0.42 & -0.42 & -0.46 & -0.46 & -0.53 & -0.58 \\
\hline 11 & $b, \bar{b}$ & & & 1.12 & 1.14 & 1.17 & 1.12 & 1.10 & 1.07 & 1.05 & 1.03 & 1.05 & 1.01 & 0.99 & 0.98 \\
\hline 12 & $\gamma, \bar{b}$ & & & 0.00 & 0.00 & 0.00 & 0.00 & 0.00 & 0.00 & 0.00 & 0.00 & 0.00 & 0.00 & 0.00 & 0.00 \\
\hline 13 & $g, \bar{b}$ & & & -0.12 & -0.12 & -0.12 & -0.13 & -0.11 & -0.12 & -0.14 & -0.11 & -0.11 & -0.13 & -0.14 & -0.14 \\
\hline 14 & $b, \gamma$ & & & 0.00 & 0.00 & 0.00 & 0.00 & 0.00 & 0.00 & 0.00 & 0.00 & 0.00 & 0.00 & 0.00 & 0.00 \\
\hline 15 & $b, g$ & & & -0.13 & -0.11 & -0.11 & -0.12 & -0.11 & -0.11 & -0.13 & -0.12 & -0.12 & -0.13 & -0.14 & -0.14 \\
\hline 16 & $\bar{c}, c$ & $\bar{u}, u$ & & 35.33 & 35.31 & 35.44 & 35.54 & 35.41 & 35.79 & 35.77 & 35.83 & 35.85 & 36.53 & 36.54 & 37.24 \\
\hline 17 & $\gamma, u$ & $\gamma, c$ & & -0.14 & -0.13 & -0.12 & -0.12 & -0.13 & -0.13 & -0.14 & -0.13 & -0.15 & -0.16 & -0.22 & -0.23 \\
\hline 18 & $g, u$ & $g, c$ & & -1.15 & -1.06 & -1.09 & -1.10 & -1.08 & -1.10 & -1.07 & -1.06 & -1.14 & -1.21 & -1.29 & -1.48 \\
\hline 19 & $\bar{c}, \gamma$ & $\bar{u}, \gamma$ & & -0.07 & -0.08 & -0.07 & -0.09 & -0.09 & -0.09 & -0.07 & -0.07 & -0.09 & -0.09 & -0.11 & -0.13 \\
\hline 20 & $\bar{c}, g$ & $\bar{u}, g$ & & -1.36 & -1.38 & -1.35 & -1.36 & -1.37 & -1.33 & -1.34 & -1.34 & -1.44 & -1.43 & -1.57 & -1.66 \\
\hline 21 & $\bar{s}, s$ & $\bar{d}, d$ & & 15.34 & 15.35 & 15.28 & 15.23 & 15.38 & 15.15 & 15.24 & 15.17 & 15.07 & 15.00 & 15.14 & 14.91 \\
\hline 22 & $\gamma, d$ & $\gamma, s$ & & 0.01 & 0.01 & 0.01 & 0.01 & 0.01 & 0.01 & 0.01 & 0.01 & 0.01 & 0.01 & 0.01 & 0.01 \\
\hline 23 & $g, d$ & $g, s$ & & -0.44 & -0.41 & -0.44 & -0.43 & -0.47 & -0.46 & -0.45 & -0.42 & -0.45 & -0.49 & -0.56 & -0.58 \\
\hline 24 & $\bar{s}, \gamma$ & $\bar{d}, \gamma$ & & 0.01 & 0.01 & 0.01 & 0.01 & 0.01 & 0.01 & 0.01 & 0.01 & 0.01 & 0.01 & 0.01 & 0.01 \\
\hline 25 & $\bar{s}, g$ & $\bar{d}, g$ & & -0.51 & -0.47 & -0.47 & -0.49 & -0.49 & -0.46 & -0.51 & -0.50 & -0.50 & -0.54 & -0.55 & -0.57 \\
\hline 26 & $\bar{b}, b$ & & & 1.14 & 1.12 & 1.10 & 1.09 & 1.08 & 1.07 & 1.06 & 1.10 & 1.12 & 1.02 & 0.99 & 1.00 \\
\hline 27 & $\gamma, b$ & & & 0.00 & 0.00 & 0.00 & 0.00 & 0.00 & 0.00 & 0.00 & 0.00 & 0.00 & 0.00 & 0.00 & 0.00 \\
\hline 28 & $\underline{g}, b$ & & & -0.13 & -0.13 & -0.11 & -0.12 & -0.10 & -0.12 & -0.12 & -0.12 & -0.12 & -0.12 & -0.10 & -0.13 \\
\hline 29 & $\bar{b}, \gamma$ & & & 0.00 & 0.00 & 0.00 & 0.00 & 0.00 & 0.00 & 0.00 & 0.00 & 0.00 & 0.00 & 0.00 & 0.00 \\
\hline 30 & $\bar{b}, g$ & & & -0.13 & -0.12 & -0.13 & -0.13 & -0.12 & -0.13 & -0.12 & -0.14 & -0.11 & -0.11 & -0.12 & -0.15 \\
\hline 31 & $\underline{\gamma}, \gamma$ & & & 3.86 & 3.86 & 3.83 & 3.84 & 3.86 & 3.83 & 3.80 & 3.80 & 3.71 & 3.75 & 3.84 & 3.77 \\
\hline 32 & $\bar{b}, \gamma$ & $\bar{s}, \gamma$ & $\bar{d}, \gamma$ & -0.03 & -0.04 & -0.04 & -0.04 & -0.04 & -0.03 & -0.04 & -0.03 & -0.03 & -0.04 & -0.04 & -0.05 \\
\hline 33 & $d, \underline{\gamma}$ & $s, \gamma$ & $b, \gamma$ & -0.04 & -0.04 & -0.02 & -0.04 & -0.04 & -0.05 & -0.05 & -0.03 & -0.05 & -0.06 & -0.05 & -0.06 \\
\hline 34 & $\gamma, \bar{b}$ & $\gamma, \bar{s}$ & $\gamma, \bar{d}$ & -0.04 & -0.03 & -0.04 & -0.03 & -0.04 & -0.03 & -0.03 & -0.04 & -0.04 & -0.04 & -0.04 & -0.05 \\
\hline \multirow[t]{2}{*}{35} & $\gamma, d$ & $\gamma, s$ & $\gamma, b$ & -0.04 & -0.05 & -0.04 & -0.04 & -0.04 & -0.04 & -0.04 & -0.04 & -0.05 & -0.05 & -0.05 & -0.07 \\
\hline & & & & 61 & 62 & 63 & 64 & 65 & 66 & 67 & 68 & 69 & 70 & 71 & 72 \\
\hline 1 & $u, \bar{u}$ & $c, \bar{c}$ & & 37.46 & 38.07 & 37.99 & 37.66 & 37.67 & 37.52 & 37.66 & 37.51 & 37.88 & 38.12 & 37.68 & 37.73 \\
\hline 2 & $\gamma, \bar{c}$ & $\gamma, \bar{u}$ & & -0.13 & -0.13 & -0.12 & -0.10 & -0.09 & -0.08 & -0.08 & -0.06 & -0.09 & -0.06 & -0.03 & -0.09 \\
\hline 3 & $g, \bar{c}$ & $g, \bar{u}$ & & -1.74 & -1.76 & -1.66 & -1.51 & -1.43 & -1.33 & -1.29 & -1.16 & -1.07 & -0.92 & -0.87 & -1.02 \\
\hline 4 & $u, \gamma$ & $c, \gamma$ & & -0.25 & -0.26 & -0.23 & -0.20 & -0.17 & -0.16 & -0.13 & -0.11 & -0.08 & -0.08 & -0.05 & -0.10 \\
\hline 5 & $u, g$ & $c, g$ & & -1.60 & -1.61 & -1.51 & -1.31 & -1.14 & -0.92 & -0.74 & -0.42 & -0.24 & 0.03 & -0.10 & -0.32 \\
\hline 6 & $d, \bar{d}$ & $s, \bar{s}$ & & 14.93 & 14.81 & 14.66 & 14.57 & 14.23 & 14.03 & 13.84 & 13.45 & 13.06 & 12.97 & 12.79 & 12.41 \\
\hline 7 & $\gamma, \bar{s}$ & $\gamma, \bar{d}$ & & 0.00 & 0.00 & 0.00 & 0.00 & 0.00 & 0.00 & 0.00 & 0.00 & 0.00 & 0.00 & 0.00 & 0.00 \\
\hline 8 & $g, \bar{s}$ & $g, \bar{d}$ & & -0.61 & -0.62 & -0.59 & -0.55 & -0.52 & -0.47 & -0.39 & -0.44 & -0.44 & -0.44 & -0.39 & -0.41 \\
\hline 9 & $d, \gamma$ & $s, \gamma$ & & 0.01 & 0.01 & 0.01 & 0.01 & 0.01 & 0.01 & 0.00 & 0.00 & 0.00 & 0.00 & 0.00 & 0.00 \\
\hline 10 & $d, g$ & $s, g$ & & -0.59 & -0.62 & -0.56 & -0.54 & -0.47 & -0.49 & -0.39 & -0.33 & -0.42 & -0.21 & -0.23 & -0.10 \\
\hline 11 & $b, \bar{b}$ & & & 0.96 & 0.91 & 0.91 & 0.82 & 0.78 & 0.71 & 0.65 & 0.65 & 0.54 & 0.44 & 0.54 & 0.49 \\
\hline 12 & $\gamma, \bar{b}$ & & & 0.00 & 0.00 & 0.00 & 0.00 & 0.00 & 0.00 & 0.00 & 0.00 & 0.00 & 0.00 & 0.00 & 0.00 \\
\hline 13 & $g, \bar{b}$ & & & -0.13 & -0.13 & -0.13 & -0.12 & -0.10 & -0.10 & -0.08 & -0.08 & -0.10 & -0.05 & -0.04 & -0.11 \\
\hline 14 & $b, \gamma$ & & & 0.00 & 0.00 & 0.00 & 0.00 & 0.00 & 0.00 & 0.00 & 0.00 & 0.00 & 0.00 & 0.00 & 0.00 \\
\hline 15 & $b, g$ & & & -0.15 & -0.14 & -0.13 & -0.13 & -0.11 & -0.09 & -0.02 & -0.07 & -0.14 & -0.06 & -0.07 & -0.05 \\
\hline 16 & $\bar{c}, c$ & $\bar{u}, u$ & & 37.76 & 37.94 & 37.71 & 37.76 & 37.71 & 37.70 & 37.39 & 37.66 & 37.70 & 36.92 & 37.88 & 38.42 \\
\hline 17 & $\gamma, u$ & $\gamma, c$ & & -0.27 & -0.25 & -0.20 & -0.20 & -0.17 & -0.15 & -0.12 & -0.13 & -0.11 & -0.11 & -0.09 & -0.15 \\
\hline 18 & $g, u$ & $g, c$ & & -1.58 & -1.62 & -1.52 & -1.31 & -1.08 & -0.89 & -0.66 & -0.61 & -0.50 & -0.39 & -0.48 & 0.86 \\
\hline 19 & $\bar{c}, \gamma$ & $\bar{u}, \gamma$ & & -0.13 & -0.13 & -0.11 & -0.10 & -0.10 & -0.08 & -0.07 & -0.06 & -0.07 & -0.07 & -0.07 & -0.04 \\
\hline 20 & $\bar{c}, g$ & $\bar{u}, g$ & & -1.74 & -1.91 & -1.66 & -1.52 & -1.45 & -1.33 & -1.21 & -1.12 & -0.89 & -0.94 & -0.83 & -1.11 \\
\hline 21 & $\bar{s}, s$ & $\bar{d}, d$ & & 14.93 & 14.83 & 14.70 & 14.50 & 14.35 & 14.12 & 13.79 & 13.36 & 13.30 & 13.22 & 12.89 & 12.25 \\
\hline 22 & $\gamma, d$ & $\gamma, s$ & & 0.01 & 0.01 & 0.01 & 0.00 & 0.01 & 0.01 & 0.01 & 0.01 & 0.00 & 0.00 & 0.00 & 0.00 \\
\hline 23 & $g, d$ & $g, s$ & & -0.54 & -0.61 & -0.57 & -0.52 & -0.46 & -0.41 & -0.37 & -0.32 & -0.36 & -0.27 & -0.23 & -0.28 \\
\hline 24 & $\bar{s}, \gamma$ & $\bar{d}, \gamma$ & & 0.01 & 0.01 & 0.01 & 0.01 & 0.00 & 0.00 & 0.00 & 0.00 & 0.00 & 0.00 & 0.00 & 0.00 \\
\hline 25 & $\bar{s}, g$ & $\bar{d}, g$ & & -0.63 & -0.64 & -0.60 & -0.58 & -0.56 & -0.48 & -0.41 & -0.29 & -0.39 & -0.38 & -0.28 & -0.13 \\
\hline 26 & $\bar{b}, b$ & & & 0.92 & 0.87 & 0.86 & 0.84 & 0.74 & 0.73 & 0.62 & 0.66 & 0.57 & 0.53 & 0.43 & 0.45 \\
\hline 27 & $\gamma, b$ & & & 0.00 & 0.00 & 0.00 & 0.00 & 0.00 & 0.00 & 0.00 & 0.00 & 0.00 & 0.00 & 0.00 & 0.02 \\
\hline 28 & $\underline{g}, b$ & & & -0.13 & -0.12 & -0.12 & -0.12 & -0.11 & -0.09 & -0.08 & -0.08 & -0.11 & -0.04 & -0.03 & -0.08 \\
\hline 29 & $\underline{\bar{b}}, \gamma$ & & & 0.00 & 0.00 & 0.00 & 0.00 & 0.00 & 0.00 & 0.00 & 0.00 & 0.00 & 0.00 & 0.00 & 0.00 \\
\hline 30 & $\bar{b}, g$ & & & -0.15 & -0.13 & -0.13 & -0.12 & -0.11 & -0.11 & -0.08 & -0.08 & -0.07 & -0.09 & -0.14 & -0.11 \\
\hline 31 & $\underline{\gamma}, \gamma$ & & & 3.65 & 3.43 & 3.19 & 2.93 & 2.72 & 2.49 & 2.31 & 2.17 & 2.11 & 1.98 & 1.81 & 1.64 \\
\hline 32 & $\bar{b}, \gamma$ & $\bar{s}, \gamma$ & $\bar{d}, \gamma$ & -0.05 & -0.05 & -0.05 & -0.04 & -0.04 & -0.04 & -0.03 & -0.02 & -0.02 & -0.03 & -0.03 & -0.02 \\
\hline 33 & $d, \underline{\gamma}$ & $s, \gamma$ & $b, \gamma$ & -0.07 & -0.06 & -0.06 & -0.05 & -0.05 & -0.04 & -0.04 & -0.04 & -0.03 & -0.03 & -0.02 & -0.05 \\
\hline 34 & $\gamma, \bar{b}$ & $\gamma, \bar{s}$ & $\gamma, \bar{d}$ & -0.06 & -0.05 & -0.05 & -0.04 & -0.04 & -0.03 & -0.03 & -0.03 & -0.03 & -0.02 & -0.02 & -0.02 \\
\hline 35 & $\gamma, d$ & $\gamma, s$ & $\gamma, b$ & -0.07 & -0.05 & -0.06 & -0.05 & -0.04 & -0.05 & -0.04 & -0.03 & -0.02 & -0.05 & -0.02 & -0.07 \\
\hline
\end{tabular}

Table 4. Same as table 2, but for the invariant mass bin $45 \mathrm{GeV}<M_{\ell \bar{\ell}}<60 \mathrm{GeV}$. 


\begin{tabular}{|c|c|c|c|c|c|c|c|c|c|c|c|c|c|c|c|}
\hline & & & & 73 & 74 & 75 & 76 & 77 & 78 & 79 & 80 & 81 & 82 & 83 & 84 \\
\hline 1 & $u, \bar{u}$ & $c, \bar{c}$ & & 22.04 & 22.07 & 22.16 & 22.20 & 22.41 & 22.54 & 22.70 & 22.87 & 22.95 & 23.15 & 23.28 & 23.45 \\
\hline 2 & $\gamma, \bar{c}$ & $\gamma, \bar{u}$ & & 0.00 & -0.01 & -0.01 & -0.01 & -0.01 & -0.01 & -0.01 & -0.01 & -0.01 & -0.01 & -0.01 & -0.01 \\
\hline 3 & $g, \bar{c}$ & $g, \bar{u}$ & & -0.95 & -0.96 & -0.96 & -0.98 & -1.00 & -1.02 & -1.02 & -1.01 & -0.98 & -0.96 & -0.93 & -0.91 \\
\hline 4 & $u, \gamma$ & $c, \gamma$ & & -0.01 & -0.01 & -0.01 & -0.01 & -0.01 & -0.01 & -0.01 & -0.01 & -0.01 & -0.01 & -0.01 & -0.01 \\
\hline 5 & $u, g$ & $c, g$ & & -0.89 & -0.90 & -0.91 & -0.93 & -0.97 & -1.02 & -1.01 & -1.00 & -0.99 & -0.98 & -0.94 & -0.90 \\
\hline 6 & $d, \bar{d}$ & $s, \bar{s}$ & & 30.85 & 30.88 & 30.83 & 30.87 & 30.86 & 30.94 & 30.88 & 30.65 & 30.47 & 30.25 & 30.02 & 29.76 \\
\hline 7 & $\gamma, \bar{s}$ & $\gamma, \bar{d}$ & & 0.00 & 0.00 & 0.00 & 0.00 & 0.00 & 0.00 & 0.00 & 0.00 & 0.00 & 0.00 & 0.00 & 0.00 \\
\hline 8 & $g, \bar{s}$ & $g, \bar{d}$ & & -1.35 & -1.35 & -1.36 & -1.39 & -1.42 & -1.47 & -1.47 & -1.44 & -1.42 & -1.36 & -1.34 & -1.28 \\
\hline 9 & $d, \gamma$ & $s, \gamma$ & & 0.00 & 0.00 & 0.00 & 0.00 & 0.00 & 0.00 & 0.00 & 0.00 & 0.00 & 0.00 & 0.00 & 0.00 \\
\hline 10 & $d, g$ & $s, g$ & & -1.32 & -1.32 & -1.33 & -1.36 & -1.42 & -1.45 & -1.46 & -1.45 & -1.42 & -1.39 & -1.36 & -1.32 \\
\hline 11 & $b, \bar{b}$ & & & 2.17 & 2.16 & 2.15 & 2.12 & 2.11 & 2.08 & 2.03 & 2.00 & 1.92 & 1.86 & 1.78 & 1.69 \\
\hline 12 & $\gamma, \bar{b}$ & & & 0.00 & 0.00 & 0.00 & 0.00 & 0.00 & 0.00 & 0.00 & 0.00 & 0.00 & 0.00 & 0.00 & 0.00 \\
\hline 13 & $g, \bar{b}$ & & & -0.32 & -0.31 & -0.31 & -0.32 & -0.32 & -0.33 & -0.33 & -0.32 & -0.31 & -0.30 & -0.28 & -0.27 \\
\hline 14 & $b, \gamma$ & & & 0.00 & 0.00 & 0.00 & 0.00 & 0.00 & 0.00 & 0.00 & 0.00 & 0.00 & 0.00 & 0.00 & 0.00 \\
\hline 15 & $b, g$ & & & -0.31 & -0.32 & -0.32 & -0.32 & -0.33 & -0.33 & -0.33 & -0.32 & -0.31 & -0.30 & -0.29 & -0.27 \\
\hline 16 & $\bar{c}, c$ & $\bar{u}, u$ & & 22.02 & 22.09 & 22.12 & 22.24 & 22.42 & 22.57 & 22.70 & 22.80 & 22.97 & 23.11 & 23.26 & 23.45 \\
\hline 17 & $\gamma, u$ & $\gamma, c$ & & -0.01 & -0.01 & -0.01 & -0.01 & -0.01 & -0.01 & -0.01 & -0.01 & -0.01 & -0.01 & -0.01 & -0.01 \\
\hline 18 & $g, u$ & $g, c$ & & -0.90 & -0.90 & -0.91 & -0.93 & -0.96 & -1.02 & -1.02 & -1.01 & -0.99 & -0.96 & -0.94 & -0.90 \\
\hline 19 & $\bar{c}, \gamma$ & $\bar{u}, \gamma$ & & -0.01 & -0.01 & -0.01 & -0.01 & -0.01 & -0.01 & -0.01 & -0.01 & -0.01 & -0.01 & -0.01 & -0.01 \\
\hline 20 & $\bar{c}, g$ & $\bar{u}, g$ & & -0.94 & -0.95 & -0.96 & -0.97 & -1.00 & -1.02 & -1.03 & -1.00 & -0.99 & -0.96 & -0.93 & -0.90 \\
\hline 21 & $\bar{s}, s$ & $\bar{d}, d$ & & 30.86 & 30.82 & 30.84 & 30.88 & 30.87 & 30.92 & 30.78 & 30.64 & 30.47 & 30.20 & 30.03 & 29.75 \\
\hline 22 & $\gamma, d$ & $\gamma, s$ & & 0.00 & 0.00 & 0.00 & 0.00 & 0.00 & 0.00 & 0.00 & 0.00 & 0.00 & 0.00 & 0.00 & 0.00 \\
\hline 23 & $g, d$ & $g, s$ & & -1.30 & -1.31 & -1.34 & -1.35 & -1.41 & -1.47 & -1.47 & -1.45 & -1.43 & -1.38 & -1.35 & -1.32 \\
\hline 24 & $\bar{s}, \gamma$ & $\bar{d}, \gamma$ & & 0.00 & 0.00 & 0.00 & 0.00 & 0.00 & 0.00 & 0.00 & 0.00 & 0.00 & 0.00 & 0.00 & 0.00 \\
\hline 25 & $\bar{s}, g$ & $\bar{d}, g$ & & -1.34 & -1.36 & -1.36 & -1.39 & -1.42 & -1.47 & -1.46 & -1.43 & -1.40 & -1.37 & -1.33 & -1.29 \\
\hline 26 & $\bar{b}, b$ & & & 2.16 & 2.16 & 2.16 & 2.12 & 2.11 & 2.08 & 2.03 & 1.98 & 1.94 & 1.85 & 1.77 & 1.71 \\
\hline 27 & $\gamma, b$ & & & 0.00 & 0.00 & 0.00 & 0.00 & 0.00 & 0.00 & 0.00 & 0.00 & 0.00 & 0.00 & 0.00 & 0.00 \\
\hline 28 & $g, b$ & & & -0.31 & -0.31 & -0.31 & -0.32 & -0.33 & -0.33 & -0.33 & -0.32 & -0.31 & -0.30 & -0.29 & -0.27 \\
\hline 29 & $\bar{b}, \gamma$ & & & 0.00 & 0.00 & 0.00 & 0.00 & 0.00 & 0.00 & 0.00 & 0.00 & 0.00 & 0.00 & 0.00 & 0.00 \\
\hline 30 & $\bar{b}, g$ & & & -0.31 & -0.32 & -0.32 & -0.32 & -0.32 & -0.33 & -0.33 & -0.32 & -0.31 & -0.30 & -0.29 & -0.27 \\
\hline 31 & $\gamma, \gamma$ & & & 0.19 & 0.19 & 0.19 & 0.19 & 0.19 & 0.19 & 0.19 & 0.18 & 0.18 & 0.17 & 0.16 & 0.15 \\
\hline 32 & $\bar{b}, \gamma$ & $\bar{s}, \gamma$ & $\bar{d}, \gamma$ & 0.00 & 0.00 & 0.00 & 0.00 & 0.00 & 0.00 & 0.00 & 0.00 & 0.00 & 0.00 & 0.00 & 0.00 \\
\hline 33 & $d, \underline{\gamma}$ & $s, \gamma$ & $b, \gamma$ & 0.00 & 0.00 & 0.00 & 0.00 & 0.00 & 0.00 & 0.00 & 0.00 & 0.00 & 0.00 & 0.00 & 0.00 \\
\hline 34 & $\gamma, \bar{b}$ & $\gamma, \bar{s}$ & $\gamma, \bar{d}$ & 0.00 & 0.00 & 0.00 & 0.00 & 0.00 & 0.00 & 0.00 & 0.00 & 0.00 & 0.00 & 0.00 & 0.00 \\
\hline \multirow[t]{2}{*}{35} & $\gamma, d$ & $\gamma, s$ & $\gamma, b$ & 0.00 & 0.00 & 0.00 & 0.00 & 0.00 & 0.00 & 0.00 & 0.00 & 0.00 & 0.00 & 0.00 & 0.00 \\
\hline & & & & 85 & 86 & 87 & 88 & 89 & 90 & 91 & 92 & 93 & 94 & 95 & 96 \\
\hline 1 & $u, \bar{u}$ & $c, \bar{c}$ & & 23.60 & 23.88 & 24.00 & 24.21 & 24.48 & 24.76 & 25.21 & 25.36 & 25.69 & 26.46 & 26.86 & 27.92 \\
\hline 2 & $\gamma, \bar{c}$ & $\gamma, \bar{u}$ & & -0.01 & -0.01 & 0.00 & 0.00 & 0.00 & 0.00 & 0.00 & 0.00 & 0.00 & 0.00 & 0.00 & 0.00 \\
\hline 3 & $g, \bar{c}$ & $g, \bar{u}$ & & -0.86 & -0.82 & -0.77 & -0.73 & -0.70 & -0.67 & -0.59 & -0.54 & -0.51 & -0.48 & -0.43 & -0.42 \\
\hline 4 & $u, \gamma$ & $c, \gamma$ & & -0.01 & -0.01 & -0.01 & -0.01 & -0.01 & -0.01 & -0.01 & -0.01 & -0.01 & 0.00 & -0.01 & 0.00 \\
\hline 5 & $u, g$ & $c, g$ & & -0.87 & -0.82 & -0.79 & -0.73 & -0.67 & -0.61 & -0.52 & -0.54 & -0.48 & -0.46 & -0.61 & -0.73 \\
\hline 6 & $d, \bar{d}$ & $s, \bar{s}$ & & 29.49 & 29.07 & 28.82 & 28.52 & 28.07 & 27.80 & 27.10 & 26.92 & 26.50 & 25.99 & 25.70 & 25.02 \\
\hline 7 & $\gamma, \bar{s}$ & $\gamma, \bar{d}$ & & 0.00 & 0.00 & 0.00 & 0.00 & 0.00 & 0.00 & 0.00 & 0.00 & 0.00 & 0.00 & 0.00 & 0.00 \\
\hline 8 & $g, \bar{s}$ & $g, \bar{d}$ & & -1.23 & -1.19 & -1.13 & -1.07 & -1.00 & -0.97 & -0.91 & -0.84 & -0.81 & -0.81 & -0.81 & -0.75 \\
\hline 9 & $d, \gamma$ & $s, \gamma$ & & 0.00 & 0.00 & 0.00 & 0.00 & 0.00 & 0.00 & 0.00 & 0.00 & 0.00 & 0.00 & 0.00 & 0.00 \\
\hline 10 & $d, g$ & $s, g$ & & -1.27 & -1.23 & -1.17 & -1.13 & -1.08 & -1.02 & -0.99 & -1.00 & -0.97 & -0.98 & -1.06 & -1.16 \\
\hline 11 & $b, \bar{b}$ & & & 1.61 & 1.52 & 1.43 & 1.31 & 1.22 & 1.13 & 1.02 & 0.96 & 0.86 & 0.75 & 0.67 & 0.60 \\
\hline 12 & $\gamma, \bar{b}$ & & & 0.00 & 0.00 & 0.00 & 0.00 & 0.00 & 0.00 & 0.00 & 0.00 & 0.00 & 0.00 & 0.00 & 0.00 \\
\hline 13 & $g, \bar{b}$ & & & -0.26 & -0.24 & -0.22 & -0.21 & -0.19 & -0.17 & -0.17 & -0.15 & -0.14 & -0.13 & -0.12 & -0.09 \\
\hline 14 & $b, \gamma$ & & & 0.00 & 0.00 & 0.00 & 0.00 & 0.00 & 0.00 & 0.00 & 0.00 & 0.00 & 0.00 & 0.00 & 0.00 \\
\hline 15 & $b, g$ & & & -0.26 & -0.25 & -0.23 & -0.21 & -0.18 & -0.18 & -0.16 & -0.14 & -0.12 & -0.12 & -0.11 & -0.09 \\
\hline 16 & $\bar{c}, c$ & $\bar{u}, u$ & & 23.61 & 23.85 & 24.00 & 24.19 & 24.52 & 24.73 & 24.91 & 25.36 & 25.73 & 25.94 & 27.07 & 27.31 \\
\hline 17 & $\gamma, u$ & $\gamma, c$ & & -0.01 & -0.01 & -0.01 & -0.01 & -0.01 & -0.01 & -0.01 & -0.01 & 0.00 & 0.00 & 0.00 & -0.01 \\
\hline 18 & $g, u$ & $g, c$ & & -0.86 & -0.82 & -0.78 & -0.73 & -0.69 & -0.62 & -0.53 & -0.54 & -0.54 & -0.45 & -0.76 & -0.74 \\
\hline 19 & $\bar{c}, \gamma$ & $\bar{u}, \gamma$ & & -0.01 & -0.01 & 0.00 & 0.00 & 0.00 & 0.00 & 0.00 & 0.00 & 0.00 & 0.00 & 0.00 & 0.00 \\
\hline 20 & $\bar{c}, g$ & $\bar{u}, g$ & & -0.86 & -0.82 & -0.77 & -0.73 & -0.70 & -0.65 & -0.59 & -0.55 & -0.51 & -0.46 & -0.47 & -0.43 \\
\hline 21 & $\bar{s}, s$ & $\bar{d}, d$ & & 29.47 & 29.14 & 28.83 & 28.54 & 28.11 & 27.64 & 27.29 & 26.91 & 26.39 & 25.97 & 25.56 & 24.97 \\
\hline 22 & $\gamma, d$ & $\gamma, s$ & & 0.00 & 0.00 & 0.00 & 0.00 & 0.00 & 0.00 & 0.00 & 0.00 & 0.00 & 0.00 & 0.00 & 0.00 \\
\hline 23 & $g, d$ & $g, s$ & & -1.27 & -1.23 & -1.18 & -1.13 & -1.08 & -1.04 & -0.95 & -1.01 & -0.94 & -1.06 & -1.19 & -1.14 \\
\hline 24 & $\bar{s}, \gamma$ & $\bar{d}, \gamma$ & & 0.00 & 0.00 & 0.00 & 0.00 & 0.00 & 0.00 & 0.00 & 0.00 & 0.00 & 0.00 & 0.00 & 0.00 \\
\hline 25 & $\bar{s}, g$ & $\bar{d}, g$ & & -1.23 & -1.19 & -1.11 & -1.08 & -1.02 & -0.97 & -0.90 & -0.90 & -0.80 & -0.75 & -0.77 & -0.73 \\
\hline 26 & $\bar{b}, b$ & & & 1.62 & 1.52 & 1.43 & 1.31 & 1.23 & 1.13 & 1.04 & 0.94 & 0.85 & 0.76 & 0.64 & 0.59 \\
\hline 27 & $\gamma, b$ & & & 0.00 & 0.00 & 0.00 & 0.00 & 0.00 & 0.00 & 0.00 & 0.00 & 0.00 & 0.00 & 0.00 & 0.00 \\
\hline 28 & $\underline{g}, b$ & & & -0.26 & -0.24 & -0.23 & -0.21 & -0.20 & -0.17 & -0.16 & -0.15 & -0.13 & -0.11 & -0.11 & -0.08 \\
\hline 29 & $\bar{b}, \gamma$ & & & 0.00 & 0.00 & 0.00 & 0.00 & 0.00 & 0.00 & 0.00 & 0.00 & 0.00 & 0.00 & 0.00 & 0.00 \\
\hline 30 & $\bar{b}, g$ & & & -0.26 & -0.24 & -0.22 & -0.20 & -0.20 & -0.18 & -0.15 & -0.15 & -0.13 & -0.12 & -0.11 & -0.10 \\
\hline 31 & $\underline{\gamma}, \gamma$ & & & 0.14 & 0.13 & 0.12 & 0.11 & 0.10 & 0.09 & 0.09 & 0.08 & 0.07 & 0.07 & 0.07 & 0.07 \\
\hline 32 & $\bar{b}, \gamma$ & $\bar{s}, \gamma$ & $\bar{d}, \gamma$ & 0.00 & 0.00 & 0.00 & 0.00 & 0.00 & 0.00 & 0.00 & 0.00 & 0.00 & 0.00 & 0.00 & 0.00 \\
\hline 33 & $d, \underline{\gamma}$ & $s, \gamma$ & $b, \gamma$ & 0.00 & 0.00 & 0.00 & 0.00 & 0.00 & 0.00 & 0.00 & 0.00 & 0.00 & 0.00 & 0.00 & 0.00 \\
\hline 34 & $\gamma, \bar{b}$ & $\gamma, \bar{s}$ & $\gamma, \bar{d}$ & 0.00 & 0.00 & 0.00 & 0.00 & 0.00 & 0.00 & 0.00 & 0.00 & 0.00 & 0.00 & 0.00 & 0.00 \\
\hline 35 & $\gamma, d$ & $\gamma, s$ & $\gamma, b$ & 0.00 & 0.00 & 0.00 & 0.00 & 0.00 & 0.00 & 0.00 & 0.00 & 0.00 & 0.00 & 0.00 & 0.00 \\
\hline
\end{tabular}

Table 5. Same as table 2, but for the invariant mass bin $60 \mathrm{GeV}<M_{\ell \bar{\ell}}<120 \mathrm{GeV}$. 


\begin{tabular}{|c|c|c|c|c|c|c|c|c|c|c|c|c|c|c|c|}
\hline & & & & 97 & 98 & 99 & 100 & 101 & 102 & 103 & 104 & 105 & 106 & 107 & 108 \\
\hline 1 & $u, \bar{u}$ & $c, \bar{c}$ & & 28.42 & 28.23 & 27.88 & 28.64 & 28.74 & 29.04 & 28.88 & 29.27 & 29.40 & 29.79 & 29.95 & 30.24 \\
\hline 2 & $\gamma, \bar{c}$ & $\gamma, \bar{u}$ & & -0.11 & -0.06 & -0.11 & -0.07 & -0.06 & -0.07 & -0.07 & -0.06 & -0.06 & -0.04 & -0.03 & -0.05 \\
\hline 3 & $g, \bar{c}$ & $g, \bar{u}$ & & -1.26 & -1.22 & -1.26 & -1.20 & -1.21 & -1.12 & -1.14 & -1.14 & -1.04 & -1.07 & -0.98 & -0.96 \\
\hline 4 & $u, \gamma$ & $c, \gamma$ & & -0.27 & -0.19 & -0.24 & -0.25 & -0.19 & -0.16 & -0.16 & -0.13 & -0.14 & -0.13 & -0.11 & -0.09 \\
\hline 5 & $u, g$ & $c, g$ & & -1.40 & -1.38 & -1.35 & -1.34 & -1.39 & -1.31 & -1.45 & -1.47 & -1.33 & -1.41 & -1.38 & -1.43 \\
\hline 6 & $d, \bar{d}$ & $s, \bar{s}$ & & 24.28 & 24.35 & 24.49 & 24.18 & 23.96 & 23.93 & 23.73 & 23.71 & 23.68 & 23.03 & 22.98 & 22.78 \\
\hline 7 & $\gamma, \bar{s}$ & $\gamma, \bar{d}$ & & 0.00 & 0.00 & 0.00 & 0.00 & 0.00 & 0.00 & 0.00 & 0.00 & 0.00 & 0.00 & 0.00 & 0.00 \\
\hline 8 & $g, \bar{s}$ & $g, \bar{d}$ & & -1.19 & -1.12 & -1.19 & -1.11 & -1.13 & -1.11 & -1.07 & -1.07 & -0.99 & -1.03 & -0.94 & -0.97 \\
\hline 9 & $d, \gamma$ & $s, \gamma$ & & 0.00 & 0.01 & 0.00 & 0.01 & 0.00 & 0.00 & 0.00 & 0.00 & 0.00 & 0.00 & 0.00 & 0.00 \\
\hline 10 & $d, g$ & $s, g$ & & -1.22 & -1.23 & -1.26 & -1.26 & -1.20 & -1.26 & -1.25 & -1.20 & -1.11 & -1.14 & -1.17 & -1.15 \\
\hline 11 & $b, \bar{b}$ & & & 1.53 & 1.53 & 1.50 & 1.47 & 1.46 & 1.43 & 1.28 & 1.29 & 1.22 & 1.21 & 1.04 & 1.08 \\
\hline 12 & $\gamma, \bar{b}$ & & & 0.00 & 0.00 & 0.00 & 0.00 & 0.00 & 0.00 & 0.00 & 0.00 & 0.00 & 0.00 & 0.00 & 0.00 \\
\hline 13 & $g, \bar{b}$ & & & -0.26 & -0.25 & -0.23 & -0.23 & -0.23 & -0.23 & -0.22 & -0.23 & -0.22 & -0.20 & -0.19 & -0.17 \\
\hline 14 & $b, \gamma$ & & & 0.00 & 0.00 & 0.00 & 0.00 & 0.00 & 0.00 & 0.00 & 0.00 & 0.00 & 0.00 & 0.00 & 0.00 \\
\hline 15 & $b, g$ & & & -0.27 & -0.22 & -0.24 & -0.23 & -0.24 & -0.25 & -0.25 & -0.23 & -0.20 & -0.19 & -0.22 & -0.21 \\
\hline 16 & $\bar{c}, c$ & $\bar{u}, u$ & & 28.34 & 28.34 & 28.66 & 28.35 & 28.64 & 28.66 & 29.26 & 29.35 & 29.28 & 29.98 & 29.85 & 30.46 \\
\hline 17 & $\gamma, u$ & $\gamma, c$ & & -0.29 & -0.24 & -0.24 & -0.20 & -0.21 & -0.18 & -0.20 & -0.13 & -0.14 & -0.14 & -0.09 & -0.10 \\
\hline 18 & $g, u$ & $g, c$ & & -1.34 & -1.40 & -1.39 & -1.36 & -1.42 & -1.41 & -1.42 & -1.29 & -1.43 & -1.40 & -1.46 & -1.39 \\
\hline 19 & $\bar{c}, \gamma$ & $\bar{u}, \gamma$ & & -0.08 & -0.12 & -0.07 & -0.09 & -0.07 & -0.07 & -0.05 & -0.06 & -0.04 & -0.05 & -0.04 & -0.05 \\
\hline 20 & $\bar{c}, g$ & $\bar{u}, g$ & & -1.24 & -1.21 & -1.27 & -1.15 & -1.21 & -1.18 & -1.12 & -1.12 & -1.08 & -0.99 & -1.06 & -0.95 \\
\hline 21 & $\bar{s}, s$ & $\bar{d}, d$ & & 24.20 & 24.16 & 24.46 & 24.09 & 24.04 & 24.02 & 23.91 & 23.63 & 23.37 & 23.09 & 23.36 & 22.59 \\
\hline 22 & $\gamma, d$ & $\gamma, s$ & & 0.01 & 0.00 & 0.00 & 0.01 & 0.00 & 0.00 & 0.01 & 0.00 & 0.00 & 0.00 & 0.00 & 0.00 \\
\hline 23 & $g, d$ & $g, s$ & & -1.31 & -1.27 & -1.25 & -1.24 & -1.16 & -1.25 & -1.16 & -1.22 & -1.26 & -1.17 & -1.09 & -1.17 \\
\hline 24 & $\bar{s}, \gamma$ & $\bar{d}, \gamma$ & & 0.00 & 0.00 & 0.00 & 0.00 & 0.00 & 0.01 & 0.01 & 0.00 & 0.00 & 0.00 & 0.00 & 0.00 \\
\hline 25 & $\bar{s}, g$ & $\bar{d}, g$ & & -1.18 & -1.17 & -1.22 & -1.13 & -1.18 & -1.13 & -1.10 & -1.08 & -0.96 & -0.95 & -1.00 & -0.92 \\
\hline 26 & $\bar{b}, b$ & & & 1.58 & 1.50 & 1.39 & 1.47 & 1.45 & 1.42 & 1.39 & 1.31 & 1.27 & 1.16 & 1.07 & 1.03 \\
\hline 27 & $\gamma, b$ & & & 0.00 & 0.00 & 0.00 & 0.00 & 0.00 & 0.00 & 0.00 & 0.00 & 0.00 & 0.00 & 0.00 & 0.00 \\
\hline 28 & $\underline{g}, b$ & & & -0.28 & -0.37 & -0.25 & -0.24 & -0.24 & -0.25 & -0.26 & -0.23 & -0.20 & -0.21 & -0.22 & -0.16 \\
\hline 29 & $\bar{b}, \gamma$ & & & 0.00 & 0.00 & 0.00 & 0.00 & 0.00 & 0.00 & 0.00 & 0.00 & 0.00 & 0.00 & 0.00 & 0.00 \\
\hline 30 & $\bar{b}, g$ & & & -0.26 & -0.26 & -0.24 & -0.25 & -0.21 & -0.27 & -0.24 & -0.24 & -0.23 & -0.19 & -0.19 & -0.18 \\
\hline 31 & $\underline{\gamma}, \gamma$ & & & 3.74 & 3.76 & 3.53 & 3.24 & 3.14 & 2.88 & 2.79 & 2.44 & 2.27 & 2.14 & 1.99 & 1.82 \\
\hline 32 & $\bar{b}, \gamma$ & $\bar{s}, \gamma$ & $\bar{d}, \gamma$ & -0.05 & -0.04 & -0.02 & -0.02 & -0.03 & -0.03 & -0.01 & -0.02 & -0.01 & -0.02 & -0.02 & -0.02 \\
\hline 33 & $d, \underline{\gamma}$ & $s, \gamma$ & $b, \gamma$ & -0.04 & -0.06 & -0.02 & -0.04 & -0.02 & -0.04 & -0.04 & -0.03 & -0.03 & -0.03 & -0.02 & -0.01 \\
\hline 34 & $\gamma, \bar{b}$ & $\gamma, \bar{s}$ & $\gamma, \bar{d}$ & -0.04 & -0.03 & -0.04 & -0.04 & -0.03 & -0.03 & -0.02 & -0.02 & -0.02 & -0.02 & -0.02 & -0.02 \\
\hline \multirow[t]{2}{*}{35} & $\gamma, d$ & $\gamma, s$ & $\gamma, b$ & -0.04 & -0.05 & -0.04 & -0.04 & -0.04 & -0.04 & -0.04 & -0.03 & -0.03 & -0.03 & -0.02 & -0.02 \\
\hline & & & & 109 & 110 & 111 & 112 & 113 & 114 & 115 & 116 & 117 & 118 & 119 & 120 \\
\hline 1 & $u, \bar{u}$ & $c, \bar{c}$ & & 30.94 & 31.28 & 31.48 & 32.26 & 32.27 & 32.58 & 31.90 & 33.85 & 34.05 & 34.76 & 35.40 & 30.06 \\
\hline 2 & $\gamma, \bar{c}$ & $\gamma, \bar{u}$ & & -0.03 & -0.03 & -0.03 & -0.02 & -0.02 & -0.01 & -0.03 & -0.01 & -0.03 & -0.01 & -0.01 & 0.00 \\
\hline 3 & $g, \bar{c}$ & $g, \bar{u}$ & & -0.90 & -0.92 & -0.73 & -0.76 & -0.60 & -0.65 & -0.54 & -0.55 & -0.47 & -0.46 & -0.31 & -0.66 \\
\hline 4 & $u, \gamma$ & $c, \gamma$ & & -0.07 & -0.09 & -0.09 & -0.05 & -0.08 & -0.06 & -0.04 & -0.02 & -0.06 & -0.06 & -0.01 & -0.07 \\
\hline 5 & $u, g$ & $c, g$ & & -1.50 & -1.37 & -1.50 & -2.48 & -1.29 & -1.65 & -1.58 & -1.61 & -1.06 & -2.59 & -2.53 & -2.58 \\
\hline 6 & $d, \bar{d}$ & $s, \bar{s}$ & & 21.89 & 21.78 & 21.73 & 21.13 & 19.88 & 20.97 & 19.38 & 19.37 & 18.22 & 18.68 & 17.68 & 16.26 \\
\hline 7 & $\gamma, \bar{s}$ & $\gamma, \bar{d}$ & & 0.00 & 0.00 & 0.00 & 0.00 & 0.00 & 0.00 & 0.00 & 0.00 & 0.00 & 0.00 & 0.00 & 0.00 \\
\hline 8 & $g, \bar{s}$ & $g, \bar{d}$ & & -0.91 & -0.88 & -0.90 & -0.73 & -0.77 & -0.70 & -0.63 & -0.48 & -0.44 & -0.59 & -0.50 & -0.57 \\
\hline 9 & $d, \gamma$ & $s, \gamma$ & & 0.00 & 0.01 & 0.00 & 0.00 & 0.00 & 0.00 & 0.00 & 0.00 & 0.00 & 0.00 & 0.00 & 0.00 \\
\hline 10 & $d, g$ & $s, g$ & & -1.09 & -1.21 & -1.11 & -1.18 & -1.03 & -1.10 & -1.32 & -0.99 & -1.23 & -1.20 & -1.55 & -1.57 \\
\hline 11 & $b, \bar{b}$ & & & 1.03 & 0.95 & 0.91 & 0.72 & 0.67 & 0.62 & 0.65 & 0.46 & 0.47 & 0.38 & 0.35 & 0.24 \\
\hline 12 & $\gamma, \bar{b}$ & & & 0.00 & 0.00 & 0.00 & 0.00 & 0.00 & 0.00 & 0.00 & 0.00 & 0.00 & 0.00 & 0.00 & 0.00 \\
\hline 13 & $g, \bar{b}$ & & & -0.15 & -0.16 & -0.16 & -0.16 & -0.08 & -0.12 & -0.08 & -0.10 & -0.10 & -0.03 & -0.05 & 0.10 \\
\hline 14 & $b, \gamma$ & & & 0.00 & 0.00 & 0.00 & 0.00 & 0.00 & 0.00 & 0.00 & 0.00 & 0.00 & 0.00 & 0.00 & 0.00 \\
\hline 15 & $b, g$ & & & -0.17 & -0.15 & -0.10 & -0.11 & -0.09 & -0.07 & -0.08 & -0.09 & -0.07 & -0.08 & -0.07 & -0.06 \\
\hline 16 & $\bar{c}, c$ & $\bar{u}, u$ & & 30.58 & 31.14 & 31.10 & 32.36 & 32.85 & 32.28 & 35.50 & 32.54 & 34.36 & 37.81 & 39.26 & 43.46 \\
\hline 17 & $\gamma, u$ & $\gamma, c$ & & -0.08 & -0.08 & -0.08 & -0.05 & -0.07 & -0.09 & -0.06 & -0.06 & -0.05 & -0.02 & -0.03 & -0.01 \\
\hline 18 & $g, u$ & $g, c$ & & -1.47 & -1.50 & -1.38 & -1.30 & -1.73 & -1.61 & -1.61 & -1.61 & -2.15 & -2.23 & -2.88 & -1.27 \\
\hline 19 & $\bar{c}, \gamma$ & $\bar{u}, \gamma$ & & -0.03 & -0.03 & -0.03 & -0.03 & -0.01 & -0.02 & -0.01 & -0.02 & -0.02 & 0.01 & -0.02 & -0.04 \\
\hline 20 & $\bar{c}, g$ & $\bar{u}, g$ & & -0.89 & -0.83 & -0.84 & -0.71 & -0.60 & -0.67 & -0.45 & -0.53 & -0.41 & -0.51 & -0.43 & -0.34 \\
\hline 21 & $\bar{s}, s$ & $\bar{d}, d$ & & 22.49 & 22.16 & 21.70 & 21.43 & 20.62 & 21.08 & 19.61 & 19.98 & 19.90 & 16.94 & 16.48 & 17.77 \\
\hline 22 & $\gamma, d$ & $\gamma, s$ & & 0.00 & 0.00 & 0.00 & 0.00 & 0.00 & 0.00 & 0.00 & 0.00 & 0.00 & 0.00 & 0.00 & 0.00 \\
\hline 23 & $g, d$ & $g, s$ & & -1.11 & -1.16 & -1.06 & -1.06 & -0.76 & -1.31 & -1.29 & -0.58 & -1.33 & -1.39 & -0.95 & -1.22 \\
\hline 24 & $\bar{s}, \gamma$ & $\bar{d}, \gamma$ & & 0.00 & 0.00 & 0.00 & 0.00 & 0.00 & 0.00 & 0.00 & 0.00 & 0.00 & 0.00 & 0.00 & 0.00 \\
\hline 25 & $\bar{s}, g$ & $\bar{d}, g$ & & -0.93 & -0.96 & -0.81 & -0.91 & -0.77 & -0.81 & -0.62 & -0.68 & -0.58 & -0.44 & -0.56 & -0.10 \\
\hline 26 & $\bar{b}, b$ & & & 1.11 & 0.93 & 0.85 & 0.78 & 0.82 & 0.55 & 0.57 & 0.45 & 0.38 & 0.38 & 0.26 & 0.20 \\
\hline 27 & $\gamma, b$ & & & 0.00 & 0.00 & 0.00 & 0.00 & 0.00 & 0.00 & 0.00 & 0.00 & 0.00 & 0.00 & 0.00 & 0.00 \\
\hline 28 & $\underline{g}, b$ & & & -0.18 & -0.20 & -0.13 & -0.15 & -0.12 & -0.09 & -0.10 & -0.08 & -0.10 & -0.01 & -0.04 & -0.05 \\
\hline 29 & $\underline{\bar{b}}, \gamma$ & & & 0.00 & 0.00 & 0.00 & 0.00 & 0.00 & 0.00 & 0.00 & 0.00 & 0.00 & 0.00 & 0.00 & 0.00 \\
\hline 30 & $\bar{b}, g$ & & & -0.13 & -0.14 & -0.18 & -0.17 & -0.12 & -0.13 & -0.09 & -0.08 & -0.02 & -0.03 & -0.07 & -0.17 \\
\hline 31 & $\underline{\gamma}, \gamma$ & & & 1.64 & 1.49 & 1.40 & 1.23 & 1.09 & 1.05 & 0.96 & 0.87 & 0.76 & 0.69 & 0.59 & 0.63 \\
\hline 32 & $\bar{b}, \gamma$ & $\bar{s}, \gamma$ & $\bar{d}, \gamma$ & 0.00 & -0.01 & -0.01 & -0.01 & -0.01 & 0.00 & -0.02 & -0.01 & -0.01 & 0.00 & -0.02 & 0.00 \\
\hline 33 & $d, \underline{\gamma}$ & $s, \gamma$ & $b, \gamma$ & -0.01 & -0.01 & -0.02 & -0.01 & -0.01 & -0.01 & -0.01 & 0.00 & -0.01 & 0.01 & 0.00 & 0.00 \\
\hline 34 & $\gamma, \bar{b}$ & $\gamma, \bar{s}$ & $\gamma, \bar{d}$ & -0.01 & -0.01 & -0.01 & -0.01 & -0.01 & -0.01 & -0.01 & 0.01 & 0.00 & -0.01 & 0.00 & -0.01 \\
\hline 35 & $\gamma, d$ & $\gamma, s$ & $\gamma, b$ & -0.02 & -0.01 & -0.01 & -0.01 & -0.01 & -0.02 & 0.00 & -0.01 & -0.01 & 0.00 & -0.01 & -0.01 \\
\hline
\end{tabular}

Table 6. Same as table 2, but for the invariant mass bin $120 \mathrm{GeV}<M_{\ell \bar{\ell}}<200 \mathrm{GeV}$. 


\begin{tabular}{|c|c|c|c|c|c|c|c|c|c|c|c|c|c|c|c|}
\hline & & & & 121 & 122 & 123 & 124 & 125 & 126 & 127 & 128 & 129 & 130 & 131 & 132 \\
\hline 1 & $u, \bar{u}$ & $c, \bar{c}$ & & 34.15 & 35.16 & 34.90 & 35.92 & 36.34 & 38.15 & 37.57 & 40.33 & 44.41 & 41.92 & 47.45 & 14.53 \\
\hline 2 & $\gamma, \bar{c}$ & $\gamma, \bar{u}$ & & -0.19 & -0.13 & -0.17 & -0.13 & -0.13 & -0.13 & -0.11 & -0.10 & -0.09 & -0.07 & -0.03 & -0.02 \\
\hline 3 & $g, \bar{c}$ & $g, \bar{u}$ & & -1.33 & -1.31 & -1.26 & -1.16 & -1.05 & -0.89 & -0.87 & -0.81 & -0.54 & -0.43 & -0.40 & -0.56 \\
\hline 4 & $u, \gamma$ & $c, \gamma$ & & -0.88 & -0.71 & -0.60 & -0.48 & -0.43 & -0.36 & -0.38 & -0.21 & -0.19 & -0.17 & -0.25 & -0.14 \\
\hline 5 & $u, g$ & $c, g$ & & -2.42 & -2.44 & -2.55 & -2.69 & -2.95 & -3.26 & -3.79 & -4.03 & -4.77 & -4.50 & -6.74 & -12.60 \\
\hline 6 & $d, \bar{d}$ & $s, \bar{s}$ & & 19.16 & 18.52 & 19.19 & 18.54 & 17.96 & 17.04 & 17.41 & 17.52 & 15.05 & 11.73 & 12.88 & 20.30 \\
\hline 7 & $\gamma, \bar{s}$ & $\gamma, \bar{d}$ & & 0.01 & 0.01 & 0.01 & 0.00 & 0.00 & 0.00 & 0.00 & 0.00 & 0.00 & 0.00 & 0.00 & 0.00 \\
\hline 8 & $g, \bar{s}$ & $g, \bar{d}$ & & -0.93 & -0.96 & -0.89 & -0.81 & -0.80 & -0.75 & -0.66 & -0.62 & -0.46 & -0.35 & -0.26 & -0.40 \\
\hline 9 & $d, \gamma$ & $s, \gamma$ & & 0.03 & 0.01 & 0.02 & 0.01 & 0.00 & 0.01 & 0.00 & 0.00 & 0.00 & 0.01 & 0.00 & 0.00 \\
\hline 10 & $d, g$ & $s, g$ & & -1.31 & -1.29 & -1.32 & -1.33 & -1.34 & -1.46 & -1.55 & -1.56 & -1.61 & -1.70 & -1.51 & -2.48 \\
\hline 11 & $b, \bar{b}$ & & & 0.93 & 0.96 & 0.94 & 0.76 & 0.69 & 0.72 & 0.56 & 0.47 & 0.12 & 0.25 & 0.27 & 0.38 \\
\hline 12 & $\gamma, \bar{b}$ & & & 0.00 & 0.00 & 0.00 & 0.00 & 0.00 & 0.00 & 0.00 & 0.00 & 0.00 & 0.00 & 0.00 & 0.00 \\
\hline 13 & $g, \bar{b}$ & & & -0.20 & -0.19 & -0.17 & -0.18 & -0.14 & -0.13 & -0.08 & -0.10 & -0.07 & 0.01 & -0.08 & -0.03 \\
\hline 14 & $b, \gamma$ & & & 0.00 & 0.00 & 0.00 & 0.00 & 0.00 & 0.00 & 0.00 & 0.00 & 0.00 & 0.00 & 0.00 & 0.00 \\
\hline 15 & $b, g$ & & & -0.21 & -0.21 & -0.18 & -0.18 & -0.14 & -0.15 & -0.11 & -0.10 & -0.06 & -0.04 & -0.02 & -0.06 \\
\hline 16 & $\bar{c}, c$ & $\bar{u}, u$ & & 34.50 & 33.48 & 33.43 & 34.37 & 36.37 & 36.80 & 38.76 & 38.93 & 38.87 & 47.33 & 41.42 & 76.10 \\
\hline 17 & $\gamma, u$ & $\gamma, c$ & & -0.87 & -0.77 & -0.61 & -0.38 & -0.46 & -0.37 & -0.28 & -0.20 & -0.18 & -0.10 & -0.18 & -0.11 \\
\hline 18 & $g, u$ & $g, c$ & & -2.54 & -2.46 & -2.47 & -2.69 & -2.81 & -3.27 & -3.75 & -4.55 & -5.03 & -5.42 & -6.43 & -8.59 \\
\hline 19 & $\bar{c}, \gamma$ & $\bar{u}, \gamma$ & & -0.18 & -0.14 & -0.14 & -0.11 & -0.12 & -0.11 & -0.09 & -0.06 & -0.07 & -0.04 & -0.06 & -0.20 \\
\hline 20 & $\bar{c}, g$ & $\bar{u}, g$ & & -1.37 & -1.31 & -1.26 & -1.14 & -1.02 & -0.94 & -0.77 & -0.71 & -0.50 & -0.40 & -0.46 & -0.42 \\
\hline 21 & $\bar{s}, s$ & $\bar{d}, d$ & & 18.52 & 18.98 & 19.09 & 18.62 & 17.91 & 17.42 & 17.13 & 15.94 & 15.27 & 12.78 & 15.34 & 16.12 \\
\hline 22 & $\gamma, d$ & $\gamma, s$ & & 0.01 & 0.04 & 0.01 & 0.01 & 0.01 & 0.01 & 0.00 & 0.00 & 0.00 & 0.00 & 0.00 & 0.00 \\
\hline 23 & $g, d$ & $g, s$ & & -1.34 & -1.32 & -1.32 & -1.26 & -1.41 & -1.42 & -1.48 & -1.76 & -1.43 & -1.79 & -1.83 & -3.24 \\
\hline 24 & $\bar{s}, \gamma$ & $\bar{d}, \gamma$ & & 0.01 & 0.01 & 0.00 & 0.01 & 0.00 & 0.00 & 0.01 & 0.00 & 0.00 & 0.00 & 0.00 & 0.00 \\
\hline 25 & $\bar{s}, g$ & $\bar{d}, g$ & & -1.02 & -0.98 & -0.95 & -0.87 & -0.76 & -0.76 & -0.61 & -0.63 & -0.55 & -0.37 & -0.30 & -0.22 \\
\hline 26 & $\bar{b}, b$ & & & 0.93 & 0.93 & 0.92 & 0.82 & 0.65 & 0.72 & 0.53 & 0.43 & 0.34 & 0.27 & 0.19 & 0.27 \\
\hline 27 & $\gamma, b$ & & & 0.00 & 0.00 & 0.00 & 0.00 & 0.00 & 0.00 & 0.01 & 0.00 & 0.00 & 0.00 & 0.00 & 0.00 \\
\hline 28 & $g, b$ & & & -0.21 & -0.19 & -0.18 & -0.16 & -0.15 & -0.13 & -0.17 & -0.10 & -0.07 & -0.04 & -0.02 & -0.02 \\
\hline 29 & $\bar{b}, \gamma$ & & & 0.00 & 0.00 & 0.00 & 0.00 & 0.00 & 0.00 & 0.00 & 0.00 & 0.00 & 0.00 & 0.00 & 0.00 \\
\hline 30 & $\bar{b}, g$ & & & -0.20 & -0.19 & -0.17 & -0.17 & -0.16 & -0.13 & -0.13 & -0.14 & -0.08 & -0.06 & -0.04 & -0.04 \\
\hline 31 & $\underline{\gamma}, \gamma$ & & & 7.33 & 6.84 & 6.03 & 4.91 & 4.09 & 3.53 & 2.95 & 2.24 & 1.79 & 1.24 & 1.22 & 1.57 \\
\hline 32 & $\bar{b}, \gamma$ & $\bar{s}, \gamma$ & $\bar{d}, \gamma$ & -0.08 & -0.08 & -0.05 & -0.05 & -0.03 & -0.04 & -0.02 & -0.04 & -0.02 & 0.01 & 0.00 & -0.02 \\
\hline 33 & $d, \underline{\gamma}$ & $s, \gamma$ & $b, \gamma$ & -0.11 & -0.11 & -0.09 & -0.09 & -0.06 & -0.05 & -0.04 & -0.05 & -0.06 & -0.02 & -0.01 & 0.00 \\
\hline 34 & $\gamma, \bar{b}$ & $\gamma, \bar{s}$ & $\gamma, \bar{d}$ & -0.09 & -0.05 & -0.07 & -0.05 & -0.04 & -0.04 & -0.02 & -0.03 & -0.02 & -0.03 & -0.05 & -0.01 \\
\hline 35 & $\gamma, d$ & $\gamma, s$ & $\gamma, b$ & -0.12 & -0.12 & -0.09 & -0.05 & -0.05 & -0.06 & -0.03 & -0.05 & -0.03 & -0.02 & -0.09 & -0.12 \\
\hline
\end{tabular}

Table 7. Same as table 2, but for the invariant mass bin $200 \mathrm{GeV}<M_{\ell \bar{\ell}}<1500 \mathrm{GeV}$. Each bin in the rapidity of the lepton pair has a width of 0.2 .

table 5 to $60 \mathrm{GeV}<M_{\ell \bar{\ell}}<120 \mathrm{GeV}$; table 6 to $120 \mathrm{GeV}<M_{\ell \bar{\ell}}<200 \mathrm{GeV}$; and table 7 to $120 \mathrm{GeV}<M_{\ell \bar{\ell}}<1500 \mathrm{GeV}$. In each table, the bins are in the rapidity of the lepton pair, $0.0<y_{\ell \bar{\ell}}<2.4$, and each of them has a width of 0.1 (0.2 in the largest invariant mass range).

- Table 8 collects the 37 parton luminosities contributing to the top-quark pair distributions measured by the ATLAS and CMS experiments at a centre-of-mass energy of $8 \mathrm{TeV}[83,84]$. The four distributions, differential in the transverse momentum of the top quark, $p_{\mathrm{T}}^{\mathrm{t}}$, in the rapidity of the top quark, $y_{\mathrm{t}}$, in the invariant mass of the top-quark pair, $m_{\mathrm{t} \overline{\mathrm{t}}}$, and in the rapidity of the top-quark pair, $y_{\mathrm{t} \overline{\mathrm{t}}}$ are shown. The bin edges are, respectively: 0, 60, 100, 150, 200, 260, 320, 400, $500 \mathrm{GeV} ; 0.0,0.4,0.8,1.2$, $1.6,2.5 ; 345,400,470,550,650,800,1100,1600 \mathrm{GeV}$; and $0.0,0.3,0.6,0.9,1.3,2.5$.

- Table 9 collects the 166 parton luminosities contributing to the $\mathrm{Z} p_{\mathrm{T}}$ distribution measured by the CMS experiment at a centre-of-mass energy of $13 \mathrm{TeV}$ [112]. The bin edges are: 20, 22, 26, 28, 32, 37, 43, 52, 65, 85, 120, 160, 190, 220, 250, 300, 400, $500,800,1500 \mathrm{GeV}$.

\section{Low statistics and complementary results}

In this appendix we collect some additional plots, in the same format of those presented in figures $2-5$, specifically: figure 7 is the same as figure 2 , but for a low-statistic MC run; 


\begin{tabular}{|c|c|c|c|c|c|c|c|c|c|c|c|c|c|c|c|c|}
\hline & & & & 1 & 2 & 3 & 4 & 5 & 6 & 7 & 8 & 1 & 2 & 3 & 4 & 5 \\
\hline 1 & $g, g$ & & & 81.13 & 81.53 & 81.12 & 79.26 & 76.04 & 71.78 & 67.44 & 61.14 & 82.86 & 82.18 & 80.60 & 78.33 & 74.18 \\
\hline 2 & $\bar{s}, g$ & $\bar{d}, g$ & & 0.10 & 0.12 & 0.17 & 0.20 & 0.29 & 0.40 & 0.34 & 0.44 & 0.17 & 0.15 & 0.10 & 0.07 & 0.04 \\
\hline 3 & $d, g$ & $s, g$ & & 0.57 & 0.52 & 0.64 & 0.78 & 1.07 & 1.37 & 1.57 & 1.91 & 0.74 & 0.73 & 0.71 & 0.64 & 0.43 \\
\hline 4 & $\bar{b}, g$ & & & 0.01 & 0.01 & 0.01 & 0.02 & 0.01 & 0.02 & 0.03 & 0.03 & 0.01 & 0.00 & 0.00 & 0.00 & 0.00 \\
\hline 5 & $b, g$ & & & 0.01 & 0.01 & 0.01 & 0.01 & 0.03 & 0.02 & 0.05 & 0.02 & 0.01 & 0.01 & 0.01 & 0.01 & 0.00 \\
\hline 6 & $\bar{c}, g$ & $\bar{u}, g$ & & 0.10 & 0.07 & 0.11 & 0.16 & 0.24 & 0.26 & 0.29 & 0.28 & 0.12 & 0.10 & 0.08 & 0.05 & 0.01 \\
\hline 7 & $u, g$ & $b, g$ & & 1.21 & 1.21 & 1.35 & 1.75 & 2.33 & 3.02 & 3.71 & 4.52 & 1.59 & 1.67 & 1.82 & 1.81 & 1.60 \\
\hline 8 & $g, \bar{s}$ & $g, \bar{d}$ & & 0.10 & 0.13 & 0.17 & 0.19 & 0.31 & 0.34 & 0.41 & 0.42 & 0.19 & 0.21 & 0.21 & 0.21 & 0.18 \\
\hline 9 & $g, d$ & $g, s$ & & 0.53 & 0.59 & 0.62 & 0.77 & 0.96 & 1.46 & 1.63 & 1.76 & 0.73 & 0.69 & 0.63 & 0.58 & 0.45 \\
\hline 10 & $g, \bar{b}$ & & & 0.02 & 0.01 & 0.01 & 0.02 & 0.02 & 0.02 & 0.03 & 0.03 & 0.02 & 0.01 & 0.02 & 0.03 & 0.02 \\
\hline 11 & $g, b$ & & & 0.01 & 0.00 & 0.02 & 0.01 & 0.02 & 0.03 & 0.04 & 0.03 & 0.02 & 0.02 & 0.01 & 0.01 & 0.01 \\
\hline 12 & $g, \bar{c}$ & $g, \bar{u}$ & & 0.07 & 0.09 & 0.12 & 0.14 & 0.23 & 0.21 & 0.33 & 0.29 & 0.15 & 0.15 & 0.16 & 0.14 & 0.13 \\
\hline 13 & $g, u$ & $g, c$ & & 1.27 & 1.15 & 1.30 & 1.82 & 2.44 & 2.91 & 3.80 & 4.55 & 1.50 & 1.31 & 1.16 & 1.01 & 0.79 \\
\hline 14 & $g, \gamma$ & & & 0.23 & 0.27 & 0.29 & 0.33 & 0.35 & 0.39 & 0.44 & 0.46 & 0.26 & 0.23 & 0.20 & 0.17 & 0.13 \\
\hline 15 & $\bar{b}, \gamma$ & $\bar{s}, \gamma$ & $\bar{d}, \gamma$ & 0.00 & 0.00 & 0.00 & 0.00 & 0.00 & 0.00 & 0.00 & 0.00 & 0.00 & 0.00 & 0.00 & 0.00 & 0.00 \\
\hline 16 & $d, \gamma$ & $s, \gamma$ & $b, \gamma$ & 0.00 & 0.00 & 0.00 & 0.00 & 0.00 & 0.00 & 0.01 & 0.00 & 0.00 & 0.00 & 0.00 & 0.00 & 0.00 \\
\hline 17 & $\bar{c}, \gamma$ & $\bar{u}, \gamma$ & & 0.00 & 0.00 & 0.00 & 0.00 & 0.00 & 0.00 & 0.00 & 0.00 & 0.00 & 0.00 & 0.00 & 0.00 & 0.00 \\
\hline 18 & $u, \gamma$ & $c, \gamma$ & & 0.00 & 0.00 & 0.00 & 0.01 & 0.01 & 0.02 & 0.01 & 0.02 & 0.00 & 0.00 & 0.00 & 0.00 & 0.00 \\
\hline 19 & $u, \bar{u}$ & $c, \bar{c}$ & & 4.32 & 4.25 & 4.19 & 4.35 & 4.77 & 5.37 & 6.17 & 7.73 & 3.54 & 4.89 & 6.78 & 9.38 & 14.60 \\
\hline 20 & $\gamma, \bar{c}$ & $\gamma, \bar{u}$ & & 0.00 & 0.00 & 0.00 & 0.00 & 0.00 & 0.00 & 0.00 & 0.00 & 0.00 & 0.00 & 0.00 & 0.00 & 0.00 \\
\hline 21 & $d, \bar{d}$ & $s, \bar{s}$ & & 2.90 & 2.82 & 2.70 & 2.77 & 2.93 & 3.22 & 3.54 & 4.10 & 2.85 & 3.41 & 4.11 & 4.87 & 5.69 \\
\hline 22 & $\gamma, \bar{s}$ & $\gamma, \bar{d}$ & & 0.00 & 0.00 & 0.00 & 0.00 & 0.00 & 0.00 & 0.00 & 0.00 & 0.00 & 0.00 & 0.00 & 0.00 & 0.00 \\
\hline 23 & $d, \underline{\gamma}$ & $s, \gamma$ & & 0.00 & 0.00 & 0.00 & 0.00 & 0.00 & 0.00 & 0.00 & 0.00 & 0.00 & 0.00 & 0.00 & 0.00 & 0.00 \\
\hline 24 & $b, \vec{b}$ & & & -0.04 & -0.03 & -0.02 & -0.01 & -0.01 & -0.01 & -0.01 & 0.00 & -0.03 & -0.03 & -0.04 & -0.04 & -0.04 \\
\hline 25 & $\gamma, \bar{b}$ & & & 0.00 & 0.00 & 0.00 & 0.00 & 0.00 & 0.00 & 0.00 & 0.00 & 0.00 & 0.00 & 0.00 & 0.00 & 0.00 \\
\hline 26 & $b, \gamma$ & & & 0.00 & 0.00 & 0.00 & 0.00 & 0.00 & 0.00 & 0.00 & 0.00 & 0.00 & 0.00 & 0.00 & 0.00 & 0.00 \\
\hline 27 & $\bar{c}, c$ & $\bar{u}, u$ & & 4.34 & 4.22 & 4.19 & 4.34 & 4.76 & 5.45 & 6.19 & 7.54 & 2.62 & 1.95 & 1.42 & 1.03 & 0.58 \\
\hline 28 & $\gamma, d$ & $\gamma, c$ & & 0.00 & 0.00 & 0.00 & 0.01 & 0.01 & 0.01 & 0.01 & 0.06 & 0.00 & 0.00 & 0.00 & 0.01 & 0.00 \\
\hline 29 & $\bar{s}, s$ & $\bar{d}, d$ & & 2.93 & 2.79 & 2.72 & 2.75 & 2.85 & 3.29 & 3.55 & 4.23 & 2.37 & 2.01 & 1.64 & 1.27 & 0.74 \\
\hline 30 & $\gamma, d$ & $\gamma, s$ & & 0.00 & 0.00 & 0.00 & 0.00 & 0.00 & 0.00 & 0.00 & 0.00 & 0.00 & 0.00 & 0.00 & 0.00 & 0.00 \\
\hline 31 & $\bar{s}, \gamma$ & $\bar{d}, \gamma$ & & 0.00 & 0.00 & 0.00 & 0.00 & 0.00 & 0.00 & 0.00 & 0.00 & 0.00 & 0.00 & 0.00 & 0.00 & 0.00 \\
\hline 32 & $\bar{b}, b$ & & & -0.04 & -0.03 & -0.02 & -0.01 & -0.01 & -0.01 & -0.01 & 0.00 & -0.02 & -0.01 & -0.01 & 0.00 & 0.00 \\
\hline 33 & $\gamma, b$ & & & 0.00 & 0.00 & 0.00 & 0.00 & 0.00 & 0.00 & 0.00 & 0.00 & 0.00 & 0.00 & 0.00 & 0.00 & 0.00 \\
\hline 34 & $\bar{b}, \gamma$ & & & 0.00 & 0.00 & 0.00 & 0.00 & 0.00 & 0.00 & 0.00 & 0.00 & 0.00 & 0.00 & 0.00 & 0.00 & 0.00 \\
\hline 35 & $\gamma, g$ & & & 0.23 & 0.26 & 0.30 & 0.33 & 0.36 & 0.40 & 0.40 & 0.45 & 0.30 & 0.33 & 0.37 & 0.42 & 0.46 \\
\hline 36 & $\gamma, \bar{b}$ & $\gamma, \bar{s}$ & $\gamma, \bar{d}$ & 0.00 & 0.00 & 0.00 & 0.00 & 0.00 & 0.00 & 0.00 & 0.01 & 0.00 & 0.00 & 0.00 & 0.00 & 0.00 \\
\hline 37 & $\gamma, \bar{d}$ & $\gamma, s$ & $\gamma, b$ & 0.00 & 0.00 & 0.00 & 0.00 & 0.00 & 0.01 & 0.00 & 0.01 & 0.00 & 0.00 & 0.00 & 0.00 & 0.00 \\
\hline & & & & & 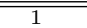 & $\bar{~} 2$ & 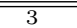 & 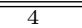 & 5 & $\overline{c 6}$ & $\overline{77}$ & 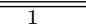 & $\bar{~} 2$ & 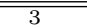 & $\overline{44}$ & $\overline{5}$ \\
\hline 1 & $g, g$ & & & & 75.95 & 79.83 & 82.25 & 83.33 & 83.66 & 83.57 & 82.79 & 84.10 & 83.37 & 81.85 & 78.93 & 69.48 \\
\hline 2 & $\bar{s}, g$ & $\bar{d}, g$ & & & 0.21 & 0.16 & 0.14 & 0.13 & 0.13 & 0.12 & 0.14 & 0.17 & 0.14 & 0.11 & 0.06 & 0.02 \\
\hline 3 & $d, g$ & $s, g$ & & & 0.73 & 0.63 & 0.60 & 0.62 & 0.66 & 0.74 & 0.81 & 0.77 & 0.78 & 0.73 & 0.65 & 0.32 \\
\hline 4 & $\bar{b}, g$ & & & & 0.02 & 0.01 & 0.01 & 0.01 & 0.01 & 0.01 & 0.01 & 0.01 & 0.01 & 0.01 & 0.00 & 0.00 \\
\hline 5 & $b, g$ & & & & 0.02 & 0.01 & 0.01 & 0.01 & 0.01 & 0.01 & 0.01 & 0.01 & 0.01 & 0.00 & 0.00 & 0.00 \\
\hline 6 & $\bar{c}, g$ & $\bar{u}, g$ & & & 0.15 & 0.11 & 0.10 & 0.09 & 0.09 & 0.09 & 0.09 & 0.13 & 0.10 & 0.07 & 0.05 & 0.00 \\
\hline 7 & $u, g$ & $b, g$ & & & 1.51 & 1.36 & 1.33 & 1.42 & 1.57 & 1.85 & 2.26 & 1.64 & 1.80 & 1.91 & 1.91 & 1.45 \\
\hline 8 & $g, \bar{s}$ & $g, \bar{d}$ & & & 0.21 & 0.16 & 0.14 & 0.13 & 0.13 & 0.12 & 0.12 & 0.20 & 0.22 & 0.23 & 0.23 & 0.16 \\
\hline 9 & $g, d$ & $g, s$ & & & 0.73 & 0.63 & 0.59 & 0.61 & 0.66 & 0.73 & 0.84 & 0.76 & 0.71 & 0.66 & 0.56 & 0.36 \\
\hline 10 & $g, \bar{b}$ & & & & 0.02 & 0.01 & 0.01 & 0.01 & 0.01 & 0.01 & 0.01 & 0.01 & 0.02 & 0.02 & 0.02 & 0.02 \\
\hline 11 & $g, b$ & & & & 0.02 & 0.01 & 0.01 & 0.01 & 0.01 & 0.01 & 0.01 & 0.01 & 0.02 & 0.02 & 0.02 & 0.02 \\
\hline 12 & $g, \bar{c}$ & $g, \bar{u}$ & & & 0.15 & 0.11 & 0.10 & 0.09 & 0.09 & 0.09 & 0.08 & 0.14 & 0.16 & 0.17 & 0.17 & 0.12 \\
\hline 13 & $g, u$ & $g, c$ & & & 1.51 & 1.36 & 1.33 & 1.42 & 1.58 & 1.85 & 2.23 & 1.48 & 1.32 & 1.15 & 0.93 & 0.59 \\
\hline 14 & $g, \gamma$ & & & & 0.25 & 0.29 & 0.29 & 0.30 & 0.31 & 0.33 & 0.35 & 0.25 & 0.22 & 0.19 & 0.17 & 0.13 \\
\hline 15 & $\bar{b}, \gamma$ & $\bar{s}, \gamma$ & & $\bar{d}, \gamma$ & 0.00 & 0.00 & 0.00 & 0.00 & 0.00 & 0.00 & 0.00 & 0.00 & 0.00 & 0.00 & 0.00 & 0.00 \\
\hline 16 & $d, \gamma$ & $s, \gamma$ & & $b, \gamma$ & 0.00 & 0.00 & 0. & 0.00 & 0.00 & 0.00 & 0.00 & 0.00 & 0.00 & 0.00 & 0.00 & 0.00 \\
\hline 17 & $\bar{c}, \gamma$ & $\bar{u}, \gamma$ & & & 0.00 & 0.00 & 0.00 & 0.00 & 0.00 & 0.00 & 0.00 & 0.00 & 0.00 & 0.00 & 0.00 & 0.00 \\
\hline 18 & $u, \gamma$ & $c, \gamma$ & & & 0.00 & 0.00 & 0. & 0.00 & 0.01 & 0.01 & 0.01 & 0.00 & 0.00 & 0.00 & 0.00 & 0.00 \\
\hline 19 & $u, \bar{u}$ & $c, \bar{c}$ & & & 5.45 & 4.54 & 3.91 & 3.59 & 3.36 & 3.34 & 3.00 & 3.04 & 4.06 & 5.64 & 8.49 & 18.00 \\
\hline 20 & $\gamma, \bar{c}$ & $\gamma, \bar{u}$ & & & 0.00 & 0.00 & 0.00 & 0.00 & 0.00 & 0.00 & 0.00 & 0.00 & 0.00 & 0.00 & 0.00 & 0.00 \\
\hline 21 & $d, \bar{d}$ & $s, \bar{s}$ & & & 3.71 & 3.00 & 2.50 & 2.18 & 2.01 & 1.83 & 1.68 & 2.52 & 3.02 & 3.68 & 4.71 & 6.99 \\
\hline 22 & $\gamma, \bar{s}$ & $\gamma, \bar{d}$ & & & 0.00 & 0.00 & 0.00 & 0.00 & 0.00 & 0.00 & 0.00 & 0.00 & 0.00 & 0.00 & 0.00 & 0.00 \\
\hline 23 & $d, \gamma$ & $s, \gamma$ & & & 0.00 & 0.00 & 0.00 & 0.00 & 0.00 & 0.00 & 0.00 & 0.00 & 0.00 & 0.00 & 0.00 & 0.00 \\
\hline 24 & $b, \bar{b}$ & & & & -0.02 & -0.02 & -0.02 & -0.02 & -0.02 & -0.02 & -0.02 & -0.03 & -0.03 & -0.02 & -0.02 & -0.02 \\
\hline 25 & $\gamma, \bar{b}$ & & & & 0.00 & 0.00 & 0.00 & 0.00 & 0.00 & 0.00 & 0.00 & 0.00 & 0.00 & 0.00 & 0.00 & 0.00 \\
\hline 26 & $b, \gamma$ & & & & 0.00 & 0.00 & & & 0.0 & 0.00 & 0.00 & 0.00 & 0.00 & 0.00 & 0.00 & 0.00 \\
\hline 27 & $\bar{c}, c$ & $\bar{u}, u$ & & & 5.45 & 4.54 & 3.93 & 3.56 & 3.42 & 3.23 & 3.45 & 2.35 & 1.86 & 1.50 & 1.20 & 0.81 \\
\hline 28 & $\gamma, d$ & $\gamma, c$ & & & 0.00 & 0.00 & 0.00 & 0.01 & 0.01 & 0.01 & 0.01 & 0.00 & 0.00 & 0.01 & 0.01 & 0.01 \\
\hline 29 & $\bar{s}, s$ & $\bar{d}, d$ & & & 3.72 & 3.00 & 2.49 & 2.21 & 1.99 & 1.77 & 1.78 & 2.16 & 1.93 & 1.73 & 1.50 & 1.01 \\
\hline 30 & $\gamma, d$ & $\gamma, s$ & & & 0.00 & 0.00 & 0.00 & 0.00 & 0.00 & 0.00 & 0.00 & 0.00 & 0.00 & 0.00 & 0.00 & 0.00 \\
\hline 31 & $\bar{s}, \gamma$ & $\bar{d}, \gamma$ & & & 0.00 & 0.00 & 0.00 & 0.00 & 0.00 & 0.00 & 0.00 & 0.00 & 0.00 & 0.00 & 0.00 & 0.00 \\
\hline 32 & $\bar{b}, b$ & & & & -0.02 & -0.02 & -0.02 & -0.02 & -0.02 & -0.02 & -0.02 & -0.03 & -0.03 & -0.02 & -0.02 & -0.02 \\
\hline 33 & $\gamma, b$ & & & & 0.00 & 0.00 & 0.00 & 0.00 & 0.00 & 0.00 & 0.00 & 0.00 & 0.00 & 0.00 & 0.00 & 0.00 \\
\hline 34 & $\bar{b}, \gamma$ & & & & 0.00 & 0.00 & 0.00 & 0.00 & 0.00 & 0.00 & 0.00 & 0.00 & 0.00 & 0.00 & 0.00 & 0.00 \\
\hline 35 & $\gamma, g$ & & & & 0.25 & 0.29 & 0.29 & 0.31 & 0.31 & 0.32 & 0.34 & 0.28 & 0.32 & 0.37 & 0.43 & 0.54 \\
\hline 36 & $\gamma, \bar{b}$ & $\gamma, \bar{s}$ & & $\gamma, \bar{d}$ & 0.00 & 0.00 & 0.00 & 0.00 & 0.00 & 0.00 & 0.00 & 0.00 & 0.00 & 0.00 & 0.00 & 0.00 \\
\hline 37 & $\gamma, \bar{d}$ & $\gamma, s$ & & $\gamma, b$ & 0.00 & 0.00 & 0.00 & 0.00 & 0.00 & 0.00 & 0.00 & 0.00 & 0.00 & 0.00 & 0.00 & 0.00 \\
\hline
\end{tabular}

Table 8. The 37 parton luminosities contributing to the predictions, accurate to NLO QCD+EW, to the top-quark pair distributions measured by the ATLAS and CMS experiments at a centre-ofmass-energy of $8 \mathrm{TeV}[83,84]$. From left to right, top to bottom, predictions are reported for the eight bins of transverse momentum of the top quark, for the five bins of top-quark rapidity, for the seven bins of top-quark pair invariant mass, and for the five bins of top-quark pair rapidity, see text for details. 


\begin{tabular}{|c|c|c|c|c|c|c|c|c|c|c|c|c|c|}
\hline Table & 9 continues into & next page & & 1 & 2 & 3 & 4 & 5 & 6 & 7 & 8 & 9 & 10 \\
\hline 1 & $g, u$ & & & 5.86 & 6.07 & 6.32 & 6.60 & 6.92 & 7.28 & 7.67 & 8.12 & 8.72 & 9.64 \\
\hline 2 & $\bar{d}, u$ & & & -0.07 & -0.07 & -0.06 & -0.03 & -0.03 & -0.01 & 0.00 & 0.03 & 0.06 & 0.10 \\
\hline 3 & $d, u$ & & & -0.09 & -0.07 & -0.05 & -0.03 & -0.01 & 0.02 & 0.07 & 0.12 & 0.19 & 0.30 \\
\hline 4 & $\bar{b}, u$ & $\bar{s}, u$ & & -0.01 & -0.01 & 0.00 & 0.00 & 0.01 & 0.01 & 0.03 & 0.03 & 0.05 & 0.06 \\
\hline 5 & $s, u$ & $b, u$ & & -0.01 & -0.01 & 0.00 & 0.00 & 0.01 & 0.02 & 0.03 & 0.04 & 0.06 & 0.09 \\
\hline 6 & $\bar{u}, u$ & & & -0.05 & -0.04 & -0.04 & -0.03 & -0.02 & -0.01 & -0.01 & 0.01 & 0.03 & 0.05 \\
\hline 7 & $u, u$ & & & -0.13 & -0.10 & -0.10 & -0.08 & -0.06 & -0.03 & 0.00 & 0.05 & 0.12 & 0.18 \\
\hline 8 & $\bar{c}, u$ & & & -0.03 & -0.03 & -0.03 & -0.02 & -0.02 & -0.01 & 0.00 & 0.01 & 0.02 & 0.03 \\
\hline 9 & $c, u$ & & & -0.04 & -0.03 & -0.03 & -0.03 & -0.02 & -0.01 & -0.01 & 0.00 & 0.01 & 0.02 \\
\hline 10 & $g, \gamma$ & & & 0.00 & -0.01 & -0.01 & 0.00 & 0.00 & 0.00 & 0.00 & 0.00 & 0.00 & 0.00 \\
\hline 11 & $g, g$ & & & -7.63 & -7.24 & -6.84 & -6.43 & -5.98 & -5.46 & -4.87 & -4.13 & -3.29 & -2.45 \\
\hline 12 & $g, c$ & & & 1.95 & 2.02 & 2.01 & 2.06 & 2.06 & 2.05 & 2.05 & 1.99 & 1.91 & 1.75 \\
\hline 13 & $\bar{b}, c$ & $\bar{d}, c$ & & -0.01 & -0.01 & -0.01 & -0.01 & 0.00 & 0.00 & 0.00 & 0.00 & 0.00 & 0.01 \\
\hline 14 & $d, c$ & $b, c$ & & -0.02 & -0.01 & -0.01 & -0.01 & -0.01 & 0.00 & 0.00 & 0.01 & 0.01 & 0.02 \\
\hline 15 & $\bar{s}, c$ & & & -0.01 & -0.01 & -0.01 & -0.01 & -0.01 & 0.00 & 0.00 & 0.00 & 0.00 & 0.00 \\
\hline 16 & $s, c$ & & & -0.01 & -0.01 & -0.01 & 0.00 & 0.00 & 0.00 & 0.00 & 0.01 & 0.01 & 0.01 \\
\hline 17 & $\bar{u}, c$ & & & -0.01 & -0.01 & -0.01 & -0.01 & -0.01 & 0.00 & 0.00 & 0.00 & 0.00 & 0.01 \\
\hline 18 & $u, c$ & & & -0.04 & -0.03 & -0.03 & -0.02 & -0.02 & -0.01 & -0.01 & 0.00 & 0.01 & 0.02 \\
\hline 19 & $\bar{c}, c$ & & & 0.00 & 0.00 & 0.00 & 0.00 & 0.00 & 0.00 & 0.00 & 0.00 & 0.00 & 0.00 \\
\hline 20 & $c, c$ & & & -0.01 & -0.01 & -0.01 & -0.01 & 0.00 & 0.00 & 0.00 & 0.00 & 0.00 & 0.00 \\
\hline 21 & $g, d$ & & & 6.23 & 6.40 & 6.64 & 6.85 & 7.13 & 7.38 & 7.67 & 8.00 & 8.31 & 8.81 \\
\hline 22 & $\vec{d}, d$ & & & -0.04 & -0.03 & -0.03 & -0.02 & -0.02 & -0.01 & 0.00 & 0.01 & 0.03 & 0.06 \\
\hline 23 & $d, d$ & & & -0.09 & -0.09 & -0.07 & -0.06 & -0.05 & -0.04 & -0.02 & 0.01 & 0.05 & 0.08 \\
\hline 24 & $\bar{b}, d$ & $\bar{s}, d$ & & -0.02 & -0.01 & -0.02 & -0.01 & -0.01 & 0.00 & 0.01 & 0.01 & 0.02 & 0.04 \\
\hline 25 & $s, d$ & $b, d$ & & -0.02 & -0.02 & -0.02 & -0.01 & -0.01 & -0.01 & 0.00 & 0.00 & 0.01 & 0.02 \\
\hline 26 & $\bar{u}, d$ & & & -0.04 & -0.04 & -0.04 & -0.03 & -0.02 & -0.01 & 0.00 & 0.01 & 0.03 & 0.05 \\
\hline 27 & $u, d$ & & & -0.09 & -0.08 & -0.05 & -0.03 & -0.01 & 0.02 & 0.06 & 0.11 & 0.20 & 0.30 \\
\hline 28 & $\bar{c}, d$ & & & -0.01 & -0.01 & -0.01 & -0.01 & 0.00 & 0.00 & 0.00 & 0.01 & 0.01 & 0.01 \\
\hline 29 & $c, d$ & & & -0.01 & -0.01 & -0.01 & -0.01 & 0.00 & 0.00 & 0.00 & 0.01 & 0.01 & 0.02 \\
\hline 30 & $g, s$ & & & 3.62 & 3.70 & 3.74 & 3.81 & 3.84 & 3.87 & 3.88 & 3.84 & 3.74 & 3.53 \\
\hline 31 & $\bar{b}, s$ & $\bar{d}, s$ & & -0.02 & -0.01 & -0.01 & -0.01 & -0.01 & -0.01 & 0.00 & 0.00 & 0.01 & 0.01 \\
\hline 32 & $d, s$ & $b, s$ & & -0.03 & -0.03 & -0.03 & -0.02 & -0.02 & -0.02 & -0.01 & 0.00 & 0.00 & 0.01 \\
\hline 33 & $\bar{s}, s$ & & & -0.01 & -0.01 & -0.01 & -0.01 & -0.01 & 0.00 & 0.00 & 0.00 & 0.00 & 0.01 \\
\hline 34 & $s, s$ & & & -0.02 & -0.02 & -0.02 & -0.02 & -0.01 & -0.01 & -0.01 & 0.00 & 0.00 & 0.00 \\
\hline 35 & $\bar{u}, s$ & & & -0.01 & -0.01 & -0.01 & -0.01 & -0.01 & -0.01 & 0.00 & 0.00 & 0.00 & 0.01 \\
\hline 36 & $u, s$ & & & -0.05 & -0.04 & -0.04 & -0.03 & -0.02 & -0.01 & -0.01 & 0.01 & 0.02 & 0.04 \\
\hline 37 & $\bar{c}, s$ & & & -0.01 & -0.01 & -0.01 & -0.01 & -0.01 & 0.00 & 0.00 & 0.00 & 0.00 & 0.01 \\
\hline 38 & $c, s$ & & & -0.01 & -0.01 & -0.01 & -0.01 & 0.00 & 0.00 & 0.00 & 0.00 & 0.01 & 0.01 \\
\hline 39 & $g, b$ & & & 1.57 & 1.63 & 1.68 & 1.73 & 1.76 & 1.79 & 1.80 & 1.78 & 1.73 & 1.62 \\
\hline 40 & $\bar{s}, b$ & $\bar{d}, b$ & & -0.01 & -0.01 & -0.01 & -0.01 & -0.01 & 0.00 & 0.00 & 0.00 & 0.00 & 0.01 \\
\hline 41 & $d, b$ & $s, b$ & & -0.02 & -0.02 & -0.02 & -0.01 & -0.01 & -0.01 & -0.01 & -0.01 & 0.00 & 0.00 \\
\hline 42 & $\bar{b}, b$ & & & 0.87 & 0.80 & 0.71 & 0.63 & 0.54 & 0.46 & 0.38 & 0.30 & 0.23 & 0.17 \\
\hline 43 & $b, b$ & & & 0.00 & 0.00 & 0.00 & 0.00 & 0.00 & 0.00 & 0.00 & 0.00 & 0.00 & 0.00 \\
\hline 44 & $\bar{c}, b$ & $\bar{u}, b$ & & -0.01 & -0.01 & -0.01 & -0.01 & 0.00 & 0.00 & 0.00 & 0.00 & 0.00 & 0.00 \\
\hline 45 & $\overrightarrow{u, b}$ & $c, b$ & & -0.02 & -0.03 & -0.02 & -0.02 & -0.01 & -0.01 & 0.00 & 0.00 & 0.01 & 0.02 \\
\hline 46 & $g, \bar{u}$ & & & 3.48 & 3.53 & 3.61 & 3.66 & 3.72 & 3.76 & 3.79 & 3.77 & 3.72 & 3.54 \\
\hline 47 & $\bar{d}, \bar{u}$ & & & -0.02 & -0.02 & -0.02 & -0.01 & -0.01 & 0.00 & 0.01 & 0.02 & 0.03 & 0.04 \\
\hline 48 & $d, \bar{u}$ & & & -0.05 & -0.04 & -0.03 & -0.03 & -0.02 & -0.01 & 0.00 & 0.01 & 0.03 & 0.05 \\
\hline 49 & $\bar{b}, \bar{u}$ & $\bar{s}, \bar{u}$ & & -0.01 & -0.01 & -0.01 & -0.01 & 0.00 & 0.00 & 0.00 & 0.01 & 0.01 & 0.02 \\
\hline 50 & $s, \bar{u}$ & $b, \bar{u}$ & & -0.01 & -0.01 & -0.01 & -0.01 & 0.00 & 0.00 & 0.00 & 0.00 & 0.01 & 0.01 \\
\hline 51 & $\bar{u}, \bar{u}$ & & & -0.02 & -0.02 & -0.02 & -0.02 & -0.01 & -0.01 & -0.01 & 0.00 & 0.00 & 0.01 \\
\hline 52 & $u, \bar{u}$ & & -0.05 & -0.04 & -0.04 & -0.03 & -0.02 & -0.01 & -0.01 & 0.00 & 0.03 & 0.05 & \\
\hline 53 & $\bar{c}, \bar{u}$ & & & -0.01 & -0.01 & -0.01 & -0.01 & -0.01 & -0.01 & 0.00 & 0.00 & 0.00 & 0.00 \\
\hline 54 & $c, \bar{u}$ & & & -0.01 & -0.01 & -0.01 & -0.01 & -0.01 & 0.00 & 0.00 & 0.00 & 0.00 & 0.00 \\
\hline 55 & $g, \bar{c}$ & & & 1.99 & 2.03 & 2.03 & 2.05 & 2.07 & 2.05 & 2.05 & 2.00 & 1.91 & 1.76 \\
\hline 56 & $\bar{b}, \bar{c}$ & $\bar{d}, \bar{c}$ & & -0.01 & -0.01 & -0.01 & 0.00 & 0.00 & 0.00 & 0.00 & 0.00 & 0.01 & 0.01 \\
\hline 57 & $d, \bar{c}$ & $b, \bar{c}$ & & -0.02 & -0.02 & -0.02 & -0.01 & -0.01 & -0.01 & 0.00 & 0.00 & 0.01 & 0.01 \\
\hline 58 & $\bar{s}, \bar{c}$ & & & -0.01 & -0.01 & 0.00 & -0.01 & 0.00 & 0.00 & 0.00 & 0.01 & 0.01 & 0.01 \\
\hline 59 & $s, \bar{c}$ & & & -0.01 & -0.01 & -0.01 & -0.01 & -0.01 & 0.00 & 0.00 & 0.00 & 0.00 & 0.01 \\
\hline 60 & $\bar{u}, \bar{c}$ & & & -0.01 & -0.01 & -0.01 & -0.01 & -0.01 & -0.01 & 0.00 & 0.00 & 0.00 & 0.00 \\
\hline 61 & $u, \bar{c}$ & & & -0.04 & -0.03 & -0.02 & -0.02 & -0.02 & -0.01 & 0.01 & 0.01 & 0.02 & 0.03 \\
\hline 62 & $\bar{c}, \bar{c}$ & & & -0.01 & -0.01 & 0.00 & -0.01 & 0.00 & 0.00 & 0.00 & 0.00 & 0.00 & 0.00 \\
\hline 63 & $c, \bar{c}$ & & & 0.00 & 0.00 & 0.00 & 0.00 & 0.00 & 0.00 & 0.00 & 0.00 & 0.00 & 0.00 \\
\hline 64 & $g, \bar{d}$ & & & 4.65 & 4.74 & 4.85 & 4.95 & 5.02 & 5.12 & 5.19 & 5.21 & 5.15 & 5.04 \\
\hline 65 & $\bar{d}, \bar{d}$ & & & -0.04 & -0.04 & -0.03 & -0.03 & -0.02 & -0.02 & -0.01 & 0.00 & 0.00 & 0.01 \\
\hline 66 & $d, \bar{d}$ & & & -0.04 & -0.04 & -0.03 & -0.03 & -0.02 & -0.01 & 0.00 & 0.01 & 0.03 & 0.05 \\
\hline 67 & $\bar{b}, \bar{d}$ & $\bar{s}, \bar{d}$ & & -0.02 & -0.02 & -0.01 & -0.01 & -0.01 & -0.01 & -0.01 & 0.00 & 0.00 & 0.00 \\
\hline 68 & $s, \bar{d}$ & $b, \bar{d}$ & & -0.02 & -0.01 & -0.01 & -0.01 & -0.01 & 0.00 & 0.00 & 0.00 & 0.01 & 0.01 \\
\hline 69 & $\bar{u}, \bar{d}$ & & & -0.02 & -0.02 & -0.02 & -0.01 & -0.01 & 0.00 & 0.01 & 0.02 & 0.03 & 0.04 \\
\hline 70 & $u, \bar{d}$ & & & -0.08 & -0.07 & -0.05 & -0.04 & -0.03 & -0.01 & 0.00 & 0.03 & 0.06 & 0.11 \\
\hline 71 & $\bar{c}, \bar{d}$ & & & -0.01 & -0.01 & -0.01 & 0.00 & 0.00 & 0.00 & 0.00 & 0.00 & 0.00 & 0.01 \\
\hline 72 & $c, \bar{d}$ & & & -0.01 & -0.01 & -0.01 & -0.01 & 0.00 & 0.00 & 0.00 & 0.00 & 0.00 & 0.01 \\
\hline 73 & $\underline{g}, \bar{s}$ & & & 3.61 & 3.67 & 3.72 & 3.78 & 3.81 & 3.83 & 3.83 & 3.76 & 3.65 & 3.39 \\
\hline 74 & $\bar{b}, \bar{s}$ & $\bar{d}, \bar{s}$ & & -0.02 & -0.02 & -0.01 & -0.02 & -0.01 & -0.01 & -0.01 & 0.00 & 0.00 & 0.00 \\
\hline 75 & $d, \bar{s}$ & $b, \bar{s}$ & & -0.03 & -0.03 & -0.02 & -0.02 & -0.02 & -0.01 & -0.01 & 0.00 & 0.01 & 0.02 \\
\hline 76 & $\bar{s}, \bar{s}$ & & & -0.02 & -0.02 & -0.02 & -0.01 & -0.01 & -0.01 & -0.01 & 0.00 & 0.00 & 0.00 \\
\hline 77 & $s, \bar{s}$ & & & -0.01 & -0.01 & -0.01 & -0.01 & -0.01 & 0.00 & 0.00 & 0.00 & 0.00 & 0.01 \\
\hline 78 & $\bar{u}, \bar{s}$ & & & -0.01 & -0.01 & -0.01 & -0.01 & -0.01 & 0.00 & 0.00 & 0.00 & 0.00 & 0.01 \\
\hline 79 & $u, \bar{s}$ & & & -0.05 & -0.04 & -0.04 & -0.03 & -0.03 & -0.02 & -0.01 & 0.00 & 0.01 & 0.03 \\
\hline 80 & $\bar{c}, \bar{s}$ & & & -0.01 & -0.01 & -0.01 & -0.01 & 0.00 & 0.00 & 0.00 & 0.00 & 0.01 & 0.01 \\
\hline 81 & $c, \bar{s}$ & & & -0.01 & -0.01 & -0.01 & -0.01 & -0.01 & 0.00 & 0.00 & 0.00 & 0.00 & 0.01 \\
\hline 82 & $g, \bar{b}$ & & & 1.60 & 1.64 & 1.68 & 1.72 & 1.76 & 1.78 & 1.80 & 1.79 & 1.73 & 1.62 \\
\hline 83 & $\bar{s}, \bar{b}$ & $\bar{d}, \bar{b}$ & & -0.01 & -0.01 & -0.01 & -0.01 & -0.01 & -0.01 & -0.01 & 0.00 & 0.00 & 0.00 \\
\hline
\end{tabular}




\begin{tabular}{|c|c|c|c|c|c|c|c|c|c|c|c|c|c|}
\hline Table & 9 continues from & previous & page into next page & 1 & 2 & 3 & 4 & 5 & 6 & 7 & 8 & 9 & 10 \\
\hline 84 & $d, \bar{b}$ & $s, \bar{b}$ & & -0.02 & -0.02 & -0.01 & -0.01 & -0.01 & -0.01 & 0.00 & 0.00 & 0.00 & 0.01 \\
\hline 85 & $\bar{b}, \bar{b}$ & & & 0.00 & 0.00 & 0.00 & 0.00 & 0.00 & 0.00 & 0.00 & 0.00 & 0.00 & 0.00 \\
\hline 86 & $b, \bar{b}$ & & & 0.89 & 0.80 & 0.71 & 0.63 & 0.54 & 0.46 & 0.38 & 0.30 & 0.23 & 0.17 \\
\hline 87 & $\bar{c}, \bar{b}$ & $\bar{u}, \bar{b}$ & & -0.01 & -0.01 & -0.01 & -0.01 & 0.00 & 0.00 & 0.00 & 0.00 & 0.00 & 0.00 \\
\hline 88 & $u, \bar{b}$ & $c, \bar{b}$ & & -0.03 & -0.02 & -0.02 & -0.02 & -0.01 & -0.01 & -0.01 & 0.00 & 0.00 & 0.01 \\
\hline 89 & $u, g$ & & & 5.86 & 6.10 & 6.34 & 6.60 & 6.93 & 7.26 & 7.66 & 8.14 & 8.71 & 9.62 \\
\hline 90 & $\gamma, g$ & & & -0.01 & -0.01 & -0.01 & -0.01 & 0.00 & 0.00 & 0.00 & 0.00 & 0.00 & 0.00 \\
\hline 91 & $u, \bar{b}$ & $u, \bar{s}$ & & -0.01 & -0.01 & 0.00 & 0.00 & 0.01 & 0.01 & 0.02 & 0.03 & 0.05 & 0.07 \\
\hline 92 & $u, s$ & $u, b$ & & -0.01 & 0.00 & 0.00 & 0.01 & 0.01 & 0.02 & 0.03 & 0.04 & 0.06 & 0.09 \\
\hline 93 & $u, \bar{u}$ & $c, \bar{c}$ & & 9.66 & 8.98 & 8.25 & 7.53 & 6.78 & 6.07 & 5.34 & 4.65 & 4.02 & 3.50 \\
\hline 94 & $\gamma, \bar{c}$ & $\gamma, \bar{u}$ & & 0.01 & 0.01 & 0.01 & 0.01 & 0.01 & 0.01 & 0.01 & 0.01 & 0.01 & 0.01 \\
\hline 95 & $g, \bar{c}$ & $g, \bar{u}$ & & -0.48 & -0.38 & -0.28 & -0.19 & -0.09 & -0.02 & 0.06 & 0.12 & 0.18 & 0.21 \\
\hline 96 & $u, \gamma$ & $c, \gamma$ & & 0.02 & 0.01 & 0.02 & 0.02 & 0.02 & 0.02 & 0.02 & 0.02 & 0.02 & 0.02 \\
\hline 97 & $u, g$ & $c, g$ & & -0.81 & -0.65 & -0.49 & -0.34 & -0.18 & -0.04 & 0.10 & 0.24 & 0.38 & 0.53 \\
\hline 98 & $c, g$ & & & 1.98 & 2.02 & 2.02 & 2.05 & 2.05 & 2.06 & 2.05 & 1.98 & 1.92 & 1.76 \\
\hline 99 & $c, \bar{b}$ & $c, \bar{d}$ & & -0.01 & -0.01 & -0.01 & -0.01 & 0.00 & 0.00 & 0.00 & 0.00 & 0.00 & 0.01 \\
\hline 100 & $c, d$ & $c, b$ & & -0.02 & -0.01 & -0.01 & -0.01 & -0.01 & 0.00 & 0.00 & 0.00 & 0.01 & 0.02 \\
\hline 101 & $d, g$ & & & 6.24 & 6.40 & 6.65 & 6.85 & 7.11 & 7.37 & 7.67 & 7.98 & 8.32 & 8.78 \\
\hline 102 & $d, \bar{b}$ & $d, \bar{s}$ & & -0.02 & -0.02 & -0.01 & -0.01 & 0.00 & 0.00 & 0.01 & 0.01 & 0.02 & 0.04 \\
\hline 103 & $d, s$ & $d, b$ & & -0.02 & -0.02 & -0.02 & -0.01 & -0.01 & -0.01 & 0.00 & 0.00 & 0.01 & 0.02 \\
\hline 104 & $d, \bar{c}$ & & & -0.01 & -0.01 & -0.01 & -0.01 & 0.00 & 0.00 & 0.00 & 0.00 & 0.01 & 0.01 \\
\hline 105 & $d, c$ & & & -0.01 & -0.01 & -0.01 & 0.00 & 0.00 & 0.00 & 0.00 & 0.01 & 0.01 & 0.02 \\
\hline 106 & $d, \bar{d}$ & $s, \bar{s}$ & & 12.68 & 11.72 & 10.70 & 9.69 & 8.63 & 7.61 & 6.58 & 5.60 & 4.68 & 3.85 \\
\hline 107 & $\gamma, \bar{s}$ & $\gamma, \bar{d}$ & & 0.00 & 0.00 & 0.00 & 0.00 & 0.00 & 0.00 & 0.00 & 0.00 & 0.00 & 0.00 \\
\hline 108 & $g, \bar{s}$ & $g, \bar{d}$ & & -0.71 & -0.56 & -0.41 & -0.28 & -0.13 & -0.01 & 0.10 & 0.20 & 0.28 & 0.34 \\
\hline 109 & $d, \gamma$ & $s, \gamma$ & & 0.00 & 0.00 & 0.00 & 0.00 & 0.00 & 0.00 & 0.00 & 0.00 & 0.00 & 0.00 \\
\hline 110 & $d, g$ & $s, g$ & & -0.91 & -0.73 & -0.54 & -0.37 & -0.19 & -0.02 & 0.13 & 0.28 & 0.42 & 0.54 \\
\hline 111 & $d, \bar{d}$ & $s, \bar{s}$ & $b, \bar{b}$ & 0.09 & 0.07 & 0.06 & 0.05 & 0.05 & 0.04 & 0.03 & 0.03 & 0.03 & 0.02 \\
\hline 112 & $g, \bar{b}$ & $g, \bar{s}$ & $g, \bar{d}$ & -0.01 & -0.01 & 0.00 & 0.00 & 0.00 & 0.00 & 0.00 & 0.00 & 0.00 & 0.00 \\
\hline 113 & $d, g$ & $s, g$ & $b, g$ & -0.01 & -0.01 & -0.01 & 0.00 & 0.00 & 0.00 & 0.00 & 0.00 & 0.00 & 0.00 \\
\hline 114 & $d, \gamma$ & $s, \gamma$ & $b, \gamma$ & 0.00 & 0.00 & 0.00 & 0.00 & 0.00 & 0.01 & 0.00 & 0.01 & 0.01 & 0.01 \\
\hline 115 & $s, g$ & & & 3.63 & 3.70 & 3.74 & 3.81 & 3.84 & 3.87 & 3.89 & 3.84 & 3.73 & 3.52 \\
\hline 116 & $s, \bar{b}$ & $s, \bar{d}$ & & -0.02 & -0.02 & -0.01 & -0.01 & -0.01 & -0.01 & 0.00 & 0.00 & 0.01 & 0.01 \\
\hline 117 & $s, d$ & $s, b$ & & -0.04 & -0.03 & -0.03 & -0.02 & -0.02 & -0.02 & -0.01 & -0.01 & 0.00 & 0.01 \\
\hline 118 & $s, \bar{u}$ & & & -0.01 & -0.01 & -0.01 & -0.01 & -0.01 & -0.01 & 0.00 & 0.00 & 0.00 & 0.01 \\
\hline 119 & $s, u$ & & & -0.04 & -0.04 & -0.04 & -0.03 & -0.02 & -0.02 & -0.01 & 0.00 & 0.02 & 0.04 \\
\hline 120 & $b, g$ & & & 1.57 & 1.64 & 1.69 & 1.72 & 1.77 & 1.78 & 1.79 & 1.78 & 1.74 & 1.62 \\
\hline 121 & $b, \bar{s}$ & $b, \bar{d}$ & & -0.01 & -0.01 & -0.01 & -0.01 & -0.01 & 0.00 & 0.00 & 0.00 & 0.00 & 0.00 \\
\hline 122 & $b, d$ & $b, s$ & & -0.02 & -0.02 & -0.02 & -0.02 & -0.01 & -0.01 & -0.01 & -0.01 & 0.00 & 0.00 \\
\hline 123 & $b, \bar{c}$ & $b, \bar{u}$ & & -0.01 & -0.01 & -0.01 & -0.01 & 0.00 & 0.00 & 0.00 & 0.00 & 0.00 & 0.00 \\
\hline 124 & $b, u$ & $b, c$ & & -0.03 & -0.02 & -0.02 & -0.01 & -0.02 & 0.00 & 0.00 & 0.00 & 0.01 & 0.02 \\
\hline 125 & $\gamma, \bar{b}$ & & & 0.00 & 0.00 & 0.00 & 0.00 & 0.00 & 0.00 & 0.00 & 0.00 & 0.00 & 0.00 \\
\hline 126 & $b, \gamma$ & & & 0.00 & 0.00 & 0.00 & 0.00 & 0.00 & 0.00 & 0.00 & 0.00 & 0.00 & 0.00 \\
\hline 127 & $\bar{u}, \underline{g}$ & & & 3.46 & 3.54 & 3.60 & 3.66 & 3.71 & 3.77 & 3.79 & 3.78 & 3.71 & 3.55 \\
\hline 128 & $\bar{u}, \bar{b}$ & $\bar{u}, \bar{s}$ & & -0.01 & -0.01 & -0.01 & -0.01 & 0.00 & 0.00 & 0.00 & 0.01 & 0.01 & 0.02 \\
\hline 129 & $\bar{u}, s$ & $\bar{u}, b$ & & -0.01 & -0.01 & -0.01 & -0.01 & 0.00 & 0.00 & 0.00 & 0.00 & 0.01 & 0.01 \\
\hline 130 & $\bar{c}, c$ & $\bar{u}, u$ & & 9.67 & 8.96 & 8.26 & 7.53 & 6.80 & 6.07 & 5.34 & 4.64 & 4.02 & 3.49 \\
\hline 131 & $\gamma, u$ & $\gamma, c$ & & 0.02 & 0.02 & 0.01 & 0.02 & 0.02 & 0.02 & 0.02 & 0.02 & 0.02 & 0.02 \\
\hline 132 & $g, u$ & $g, c$ & & -0.81 & -0.64 & -0.49 & -0.35 & -0.19 & -0.04 & 0.10 & 0.24 & 0.38 & 0.53 \\
\hline 133 & $\bar{c}, \gamma$ & $\bar{u}, \gamma$ & & 0.01 & 0.01 & 0.01 & 0.01 & 0.01 & 0.01 & 0.01 & 0.01 & 0.01 & 0.01 \\
\hline 134 & $\bar{c}, g$ & $\bar{u}, g$ & & -0.48 & -0.38 & -0.28 & -0.19 & -0.10 & -0.01 & 0.06 & 0.13 & 0.17 & 0.21 \\
\hline 135 & $\bar{c}, g$ & & & 1.98 & 2.02 & 2.03 & 2.04 & 2.06 & 2.06 & 2.04 & 2.00 & 1.91 & 1.75 \\
\hline 136 & $\bar{c}, \bar{b}$ & $\bar{c}, \bar{d}$ & & -0.01 & -0.01 & -0.01 & 0.00 & 0.00 & 0.00 & 0.00 & 0.00 & 0.01 & 0.01 \\
\hline 137 & $\bar{c}, d$ & $\bar{c}, b$ & & -0.02 & -0.02 & -0.01 & -0.01 & -0.01 & 0.00 & 0.00 & 0.00 & 0.01 & 0.01 \\
\hline 138 & $\bar{d}, g$ & & & 4.66 & 4.72 & 4.85 & 4.95 & 5.03 & 5.12 & 5.18 & 5.20 & 5.18 & 5.03 \\
\hline 139 & $\bar{d}, \bar{b}$ & $\bar{d}, \bar{s}$ & & -0.01 & -0.02 & -0.02 & -0.01 & -0.01 & -0.01 & -0.01 & 0.00 & 0.00 & 0.00 \\
\hline 140 & $\bar{d}, s$ & $\bar{d}, b$ & & -0.02 & -0.01 & -0.01 & -0.01 & -0.01 & -0.01 & 0.00 & 0.00 & 0.01 & 0.01 \\
\hline 141 & $\bar{d}, \bar{c}$ & & & -0.01 & -0.01 & -0.01 & 0.00 & 0.00 & 0.00 & 0.00 & 0.00 & 0.00 & 0.01 \\
\hline 142 & $\bar{d}, c$ & & & -0.01 & -0.01 & -0.01 & -0.01 & 0.00 & 0.00 & 0.00 & 0.00 & 0.00 & 0.00 \\
\hline 143 & $\bar{s}, s$ & $\bar{d}, d$ & & 12.67 & 11.72 & 10.67 & 9.68 & 8.65 & 7.60 & 6.57 & 5.60 & 4.68 & 3.86 \\
\hline 144 & $\gamma, d$ & $\gamma, s$ & & 0.00 & 0.00 & 0.00 & 0.00 & 0.00 & 0.00 & 0.00 & 0.00 & 0.00 & 0.00 \\
\hline 145 & $g, d$ & $g, s$ & & -0.90 & -0.73 & -0.55 & -0.36 & -0.18 & -0.02 & 0.13 & 0.28 & 0.41 & 0.54 \\
\hline 146 & $\bar{s}, \gamma$ & $\bar{d}, \gamma$ & & 0.00 & 0.00 & 0.00 & 0.00 & 0.00 & 0.00 & 0.00 & 0.00 & 0.00 & 0.00 \\
\hline 147 & $\bar{s}, g$ & $\bar{d}, g$ & & -0.72 & -0.57 & -0.41 & -0.27 & -0.14 & -0.01 & 0.10 & 0.20 & 0.28 & 0.34 \\
\hline 148 & $\bar{b}, b$ & $\bar{s}, s$ & $\bar{d}, d$ & 0.09 & 0.07 & 0.06 & 0.05 & 0.05 & 0.04 & 0.03 & 0.03 & 0.03 & 0.02 \\
\hline 149 & $g, d$ & $g, s$ & $g, b$ & -0.01 & -0.01 & -0.01 & 0.00 & 0.00 & 0.00 & 0.00 & 0.00 & 0.00 & 0.00 \\
\hline 150 & $\bar{b}, g$ & $\bar{s}, g$ & $\bar{d}, g$ & -0.01 & -0.01 & 0.00 & 0.00 & 0.00 & 0.00 & 0.00 & 0.00 & 0.00 & 0.00 \\
\hline 151 & $\bar{b}, \gamma$ & $\bar{s}, \gamma$ & $\bar{d}, \gamma$ & 0.00 & 0.00 & 0.00 & 0.00 & 0.00 & 0.00 & 0.00 & 0.00 & 0.00 & 0.00 \\
\hline 152 & $\bar{s}, g$ & & & 3.62 & 3.67 & 3.73 & 3.77 & 3.81 & 3.84 & 3.82 & 3.77 & 3.65 & 3.39 \\
\hline 153 & $\bar{s}, \bar{b}$ & $\bar{s}, \bar{d}$ & & -0.02 & -0.02 & -0.02 & -0.01 & -0.01 & -0.01 & -0.01 & 0.00 & 0.00 & 0.00 \\
\hline 154 & $\bar{s}, d$ & $\bar{s}, b$ & & -0.03 & -0.03 & -0.03 & -0.02 & -0.02 & -0.01 & 0.00 & 0.00 & 0.01 & 0.02 \\
\hline 155 & $\bar{s}, \bar{u}$ & & & -0.01 & -0.01 & -0.01 & -0.01 & 0.00 & 0.00 & 0.00 & 0.00 & 0.00 & 0.01 \\
\hline 156 & $\bar{s}, u$ & & & -0.05 & -0.04 & -0.04 & -0.03 & -0.03 & -0.02 & -0.02 & 0.00 & 0.01 & 0.03 \\
\hline 157 & $\bar{b}, g$ & & & 1.60 & 1.62 & 1.70 & 1.72 & 1.77 & 1.79 & 1.80 & 1.78 & 1.73 & 1.62 \\
\hline 158 & $\bar{b}, \bar{s}$ & $\bar{b}, \bar{d}$ & & -0.01 & -0.01 & -0.01 & -0.01 & -0.01 & -0.01 & 0.00 & 0.00 & 0.00 & 0.00 \\
\hline 159 & $\bar{b}, d$ & $\bar{b}, s$ & & -0.02 & -0.01 & -0.01 & -0.01 & -0.01 & -0.01 & 0.00 & 0.00 & 0.01 & 0.01 \\
\hline 160 & $\bar{b}, \bar{c}$ & $\bar{b}, \bar{u}$ & & -0.01 & -0.01 & -0.01 & -0.01 & 0.00 & 0.00 & 0.00 & 0.00 & 0.00 & 0.00 \\
\hline 161 & $\bar{b}, u$ & $\bar{b}, c$ & & -0.03 & -0.03 & -0.02 & -0.02 & -0.02 & -0.01 & -0.01 & 0.00 & 0.01 & 0.01 \\
\hline 162 & $\gamma, b$ & & & 0.00 & 0.00 & 0.00 & 0.00 & 0.00 & 0.00 & 0.00 & 0.00 & 0.00 & 0.00 \\
\hline 163 & $\bar{b}, \gamma$ & & & 0.00 & 0.00 & 0.00 & 0.00 & 0.00 & 0.00 & 0.00 & 0.00 & 0.00 & 0.00 \\
\hline 164 & $\gamma, d$ & $\gamma, s$ & $\gamma, b$ & 0.00 & 0.00 & 0.00 & 0.00 & 0.00 & 0.01 & 0.01 & 0.01 & 0.01 & 0.01 \\
\hline 165 & $\gamma, \bar{b}$ & $\gamma, \bar{s}$ & $\gamma, \bar{d}$ & 0.00 & 0.00 & 0.00 & 0.00 & 0.00 & 0.00 & 0.00 & 0.00 & 0.00 & 0.00 \\
\hline 166 & $\gamma, \gamma$ & & & 0.00 & 0.00 & 0.00 & 0.00 & 0.00 & 0.00 & 0.00 & 0.00 & 0.00 & 0.00 \\
\hline
\end{tabular}




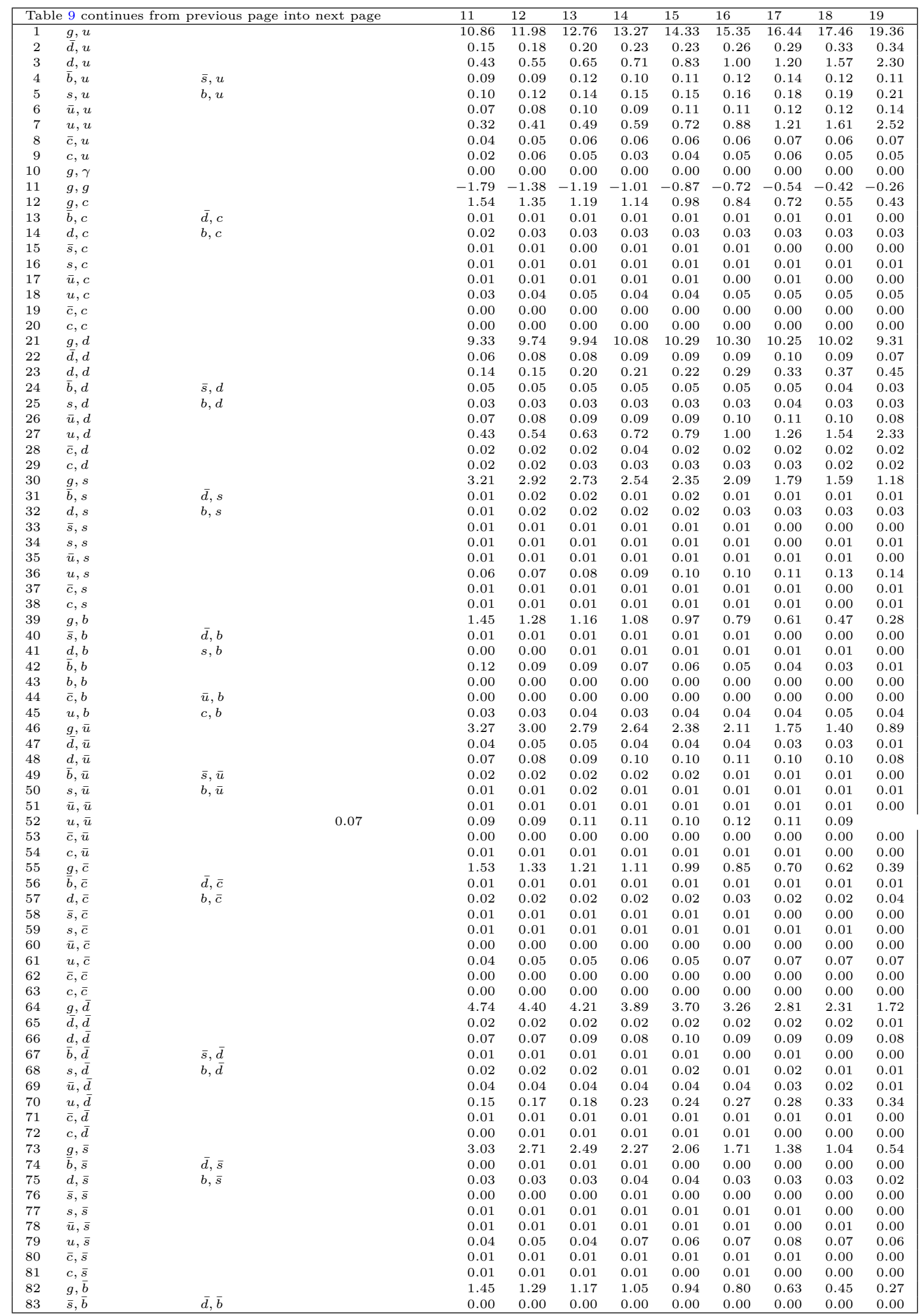




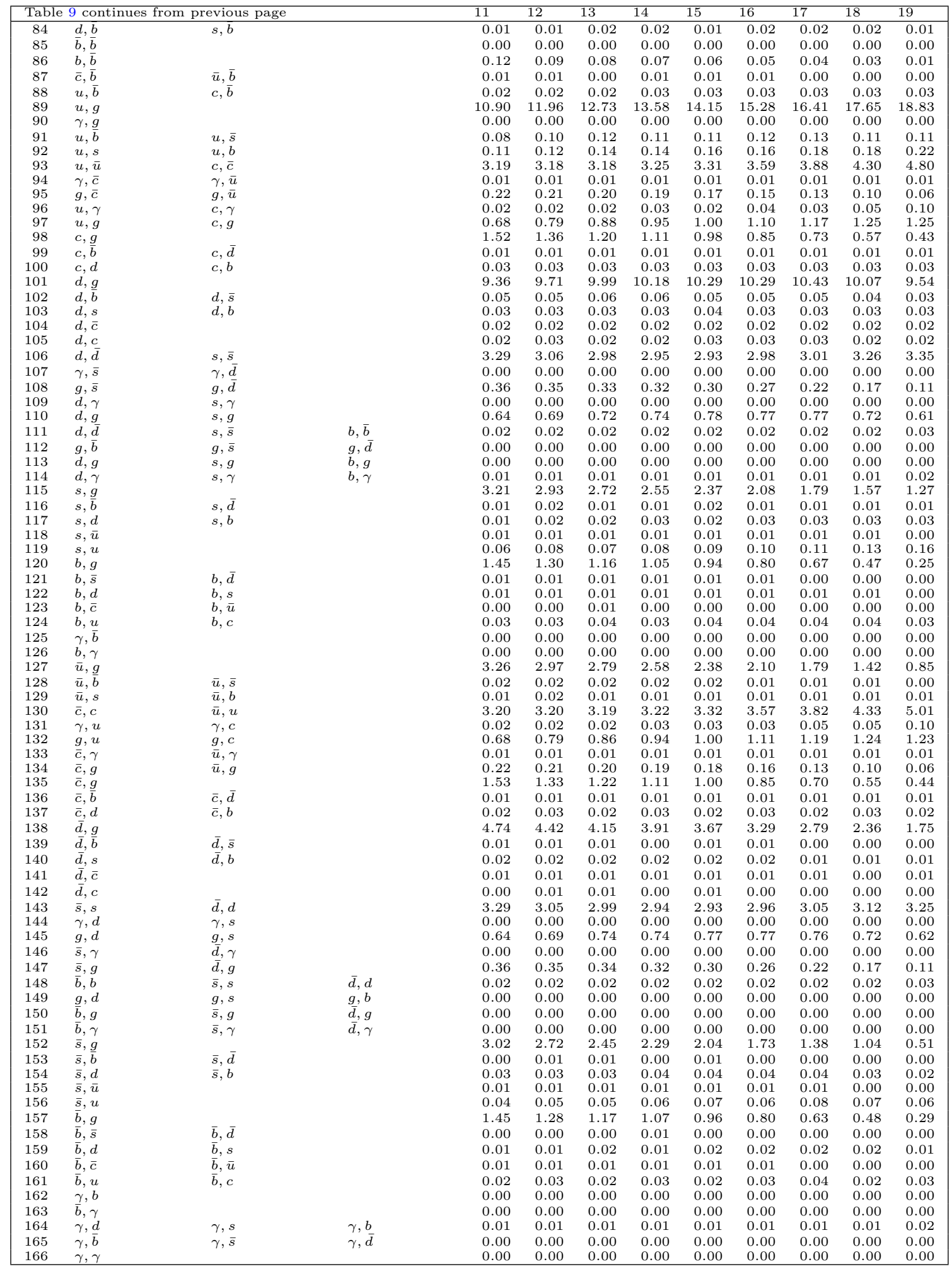

Table 9. The 166 parton luminosities contributing to the predictions, accurate to NLO QCD+EW, to the $\mathrm{Z} p_{\mathrm{T}}$ distribution measured by the CMS experiment at a centre-of-mass energy of $13 \mathrm{TeV}$ [112]. The bin edges are: 20, 22, 26, 28, 32, 37, 43, 52, 65, 85, 120, 160, 190, 220, 250, 300, 400, 500, 800, $1500 \mathrm{GeV}$. 


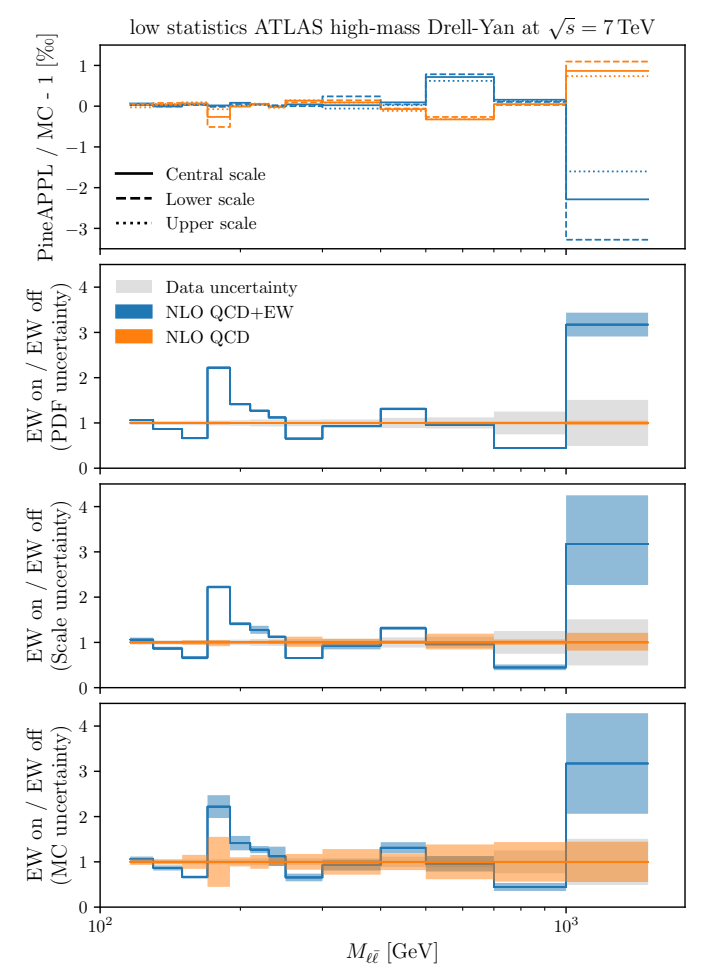

Figure 7. Same as figure 2, but for a low-statistics MC run.

figure 8 is the same as figure 3, but for the two missing lepton-pair invariant mass bins, $20 \mathrm{GeV}<M_{\ell \bar{\ell}}<30 \mathrm{GeV}$ and $30 \mathrm{GeV}<M_{\ell \bar{\ell}}<45 \mathrm{GeV}$; and figure 9 is the same as figure 4, but for the distributions in the rapidity of the top quark, $y_{\mathrm{t}}$, and in the rapidity of the top-quark pair, $y_{\mathrm{t} \overline{\mathrm{t}}}$. In this case the factorisation and renormalisation scales are kept equal to $H_{\mathrm{T}} / 4$, see section 3.2.2.

From figure 7 , we observe that the validation of the PINEAPPL result remains successful: its difference with respect to the MC result is at most $3 \%$, as usual irrespective of the accuracy of the theory and of the choice of scale. As expected, however, the result is largely unreliable to make any conclusion about the size of the EW corrections: large fluctuations are seen in the predictions, and the MC uncertainty dominates over the PDF and scale uncertainties.

Figure 8 demonstrates that the two bins at the lowest invariant mass of the Drell-Yan lepton-pair measured by the CMS experiment display very similar features as the bin at immediately larger values of invariant mass, see figure 3: the EW correction enhances the cross section by $2 \%$ to $3 \%$ across all the rapidity range; the amount of this shift is slightly larger than the PDF uncertainty, but is largely overshot by the scale uncertainty; the MC uncertainty remains comparatively negligible.

Finally, figure 9 allows us to further validate the PINEAPPL result against the MC result for the top-quark rapidity distributions, and to explicitly check that the size of the EW corrections in this case is negligible with respect to the companion top-quark transverse momentum and top-quark pair invariant mass distributions, see figure 4, consistently with what was already observed in ref. [88]. 

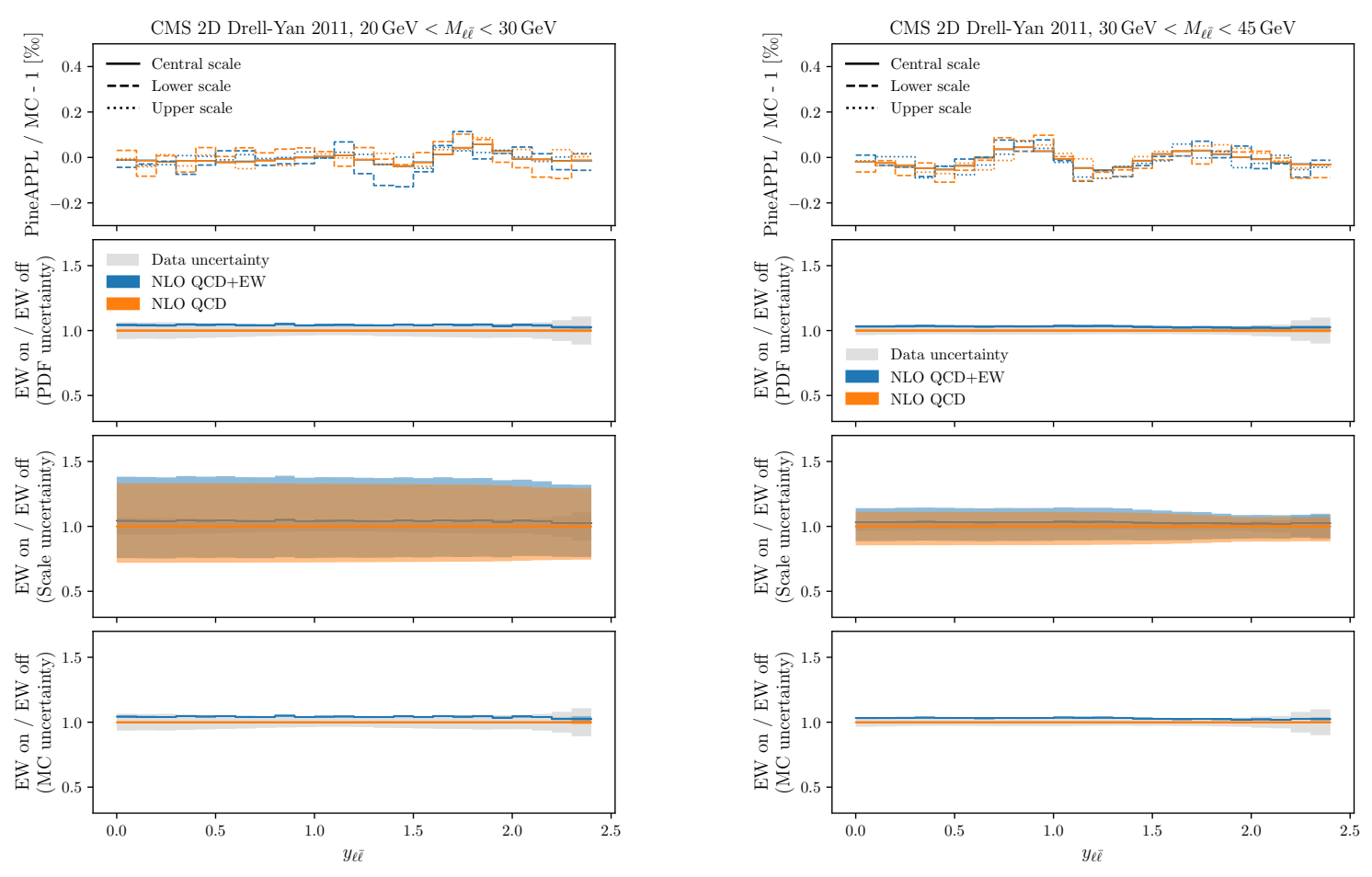

Figure 8. Same as figure 3, but for the two missing lepton-pair invariant mass bins, $20 \mathrm{GeV}<$ $M_{\ell \bar{\ell}}<30 \mathrm{GeV}$ and $30 \mathrm{GeV}<M_{\ell \bar{\ell}}<45 \mathrm{GeV}$.
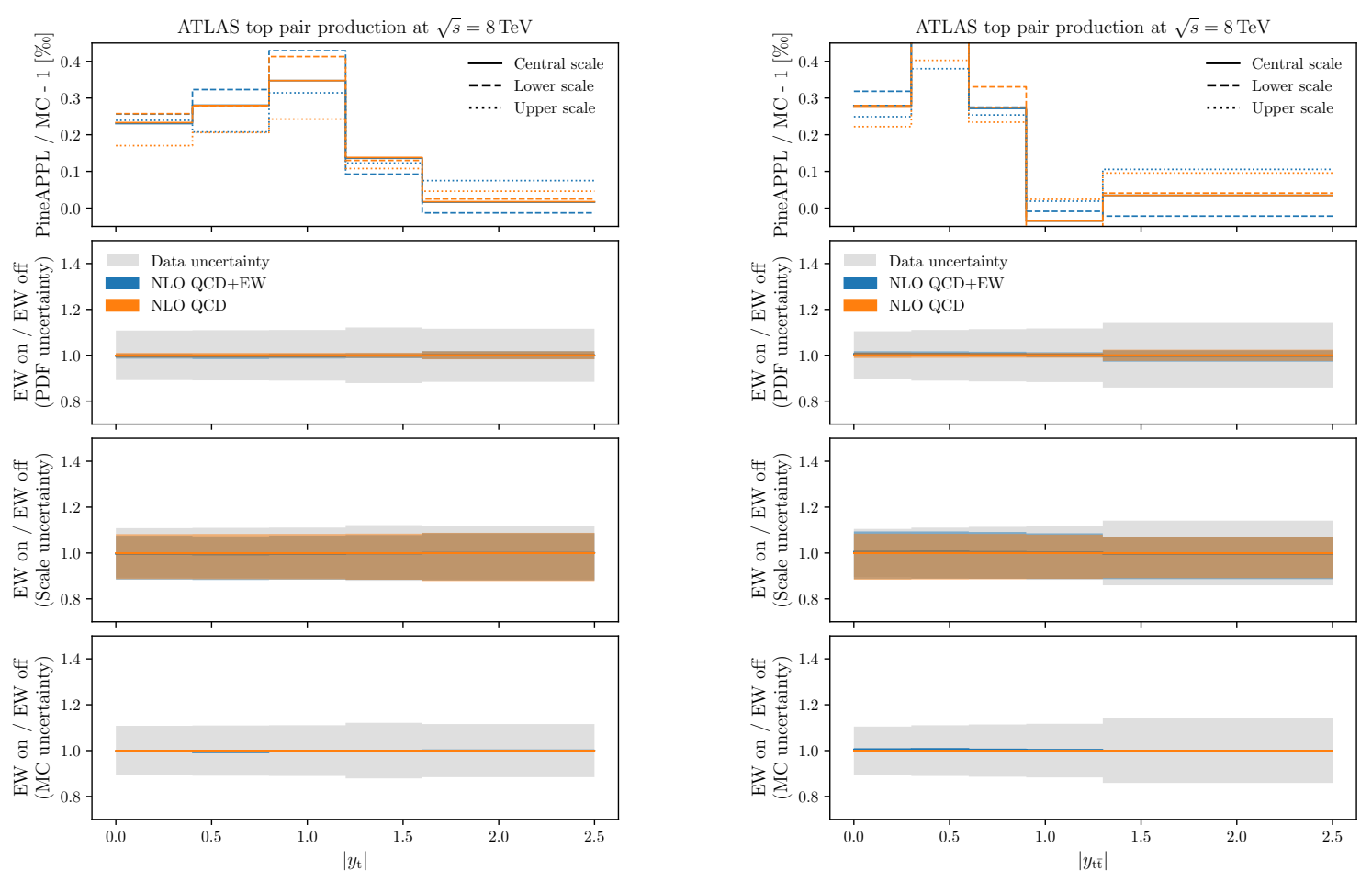

Figure 9. Same as figure 4, but for the distributions in the rapidity of the top quark, $y_{\mathrm{t}}$, and in the rapidity of the top-quark pair, $y_{\mathrm{t} \overline{\mathrm{t}}}$. 
Open Access. This article is distributed under the terms of the Creative Commons Attribution License (CC-BY 4.0), which permits any use, distribution and reproduction in any medium, provided the original author(s) and source are credited.

\section{References}

[1] M. Mangano, LHC at 10: the physics legacy, CERN Cour. 60 (2020) 40 [arXiv: 2003. 05976] [INSPIRE].

[2] J. Gao, L. Harland-Lang and J. Rojo, The structure of the proton in the LHC precision era, Phys. Rept. 742 (2018) 1 [arXiv: 1709.04922] [InSPIRE].

[3] J.J. Ethier and E.R. Nocera, Parton distributions in nucleons and nuclei, Ann. Rev. Nucl. Part. Sci. (2020) 1 [arXiv:2001.07722] [INSPIRE].

[4] T. Gleisberg et al., Event generation with SHERPA 1.1, JHEP 02 (2009) 007 [arXiv:0811.4622] [INSPIRE].

[5] J. Alwall et al., The automated computation of tree-level and next-to-leading order differential cross sections, and their matching to parton shower simulations, JHEP $\mathbf{0 7}$ (2014) 079 [arXiv: 1405.0301] [inSPIRE].

[6] J. Bellm et al., HERWIG 7.0/HERWIG++ 3.0 release note, Eur. Phys. J. C 76 (2016) 196 [arXiv:1512.01178] [INSPIRE].

[7] A. Buckley et al., General-purpose event generators for LHC physics, Phys. Rept. 504 (2011) 145 [arXiv: 1101.2599] [INSPIRE].

[8] S. Amoroso et al., Les Houches 2019: physics at TeV colliders. Standard Model working group report, in $11^{\text {th }}$ Les Houches workshop on physics at TeV colliders: PhysTeV Les Houches, (2020) [arXiv:2003.01700] [INSPIRE].

[9] S. Kallweit, J.M. Lindert, P. Maierhöfer, S. Pozzorini and M. Schönherr, NLO electroweak automation and precise predictions for W+multijet production at the LHC, JHEP 04 (2015) 012 [arXiv: 1412.5157] [INSPIRE].

[10] B. Biedermann, S. Bräuer, A. Denner, M. Pellen, S. Schumann and J.M. Thompson, Automation of NLO QCD and EW corrections with Sherpa and Recola, Eur. Phys. J. C 77 (2017) 492 [arXiv: 1704.05783] [INSPIRE].

[11] R. Frederix, S. Frixione, V. Hirschi, D. Pagani, H.-S. Shao and M. Zaro, The automation of next-to-leading order electroweak calculations, JHEP 07 (2018) 185 [arXiv:1804.10017] [INSPIRE].

[12] A. Denner and S. Dittmaier, Electroweak radiative corrections for collider physics, Phys. Rept. 864 (2020) 1 [arXiv:1912.06823] [INSPIRE].

[13] L.A. Harland-Lang, A.D. Martin, P. Motylinski and R.S. Thorne, Parton distributions in the LHC era: MMHT 2014 PDFs, Eur. Phys. J. C 75 (2015) 204 [arXiv:1412.3989] [INSPIRE].

[14] NNPDF collaboration, Parton distributions from high-precision collider data, Eur. Phys. J. C 77 (2017) 663 [arXiv:1706.00428] [INSPIRE].

[15] T.-J. Hou et al., New CTEQ global analysis of quantum chromodynamics with high-precision data from the LHC, arXiv:1912.10053 [INSPIRE]. 
[16] A.D. Martin, R.G. Roberts, W.J. Stirling and R.S. Thorne, Parton distributions incorporating QED contributions, Eur. Phys. J. C 39 (2005) 155 [hep-ph/0411040] [INSPIRE].

[17] NNPDF collaboration, Parton distributions with QED corrections, Nucl. Phys. B 877 (2013) 290 [arXiv: 1308.0598] [INSPIRE].

[18] C. Schmidt, J. Pumplin, D. Stump and C.P. Yuan, CT14QED parton distribution functions from isolated photon production in deep inelastic scattering, Phys. Rev. D 93 (2016) 114015 [arXiv: 1509.02905] [INSPIRE].

[19] A. Manohar, P. Nason, G.P. Salam and G. Zanderighi, How bright is the proton? A precise determination of the photon parton distribution function, Phys. Rev. Lett. 117 (2016) 242002 [arXiv: 1607.04266] [INSPIRE].

[20] A.V. Manohar, P. Nason, G.P. Salam and G. Zanderighi, The photon content of the proton, JHEP 12 (2017) 046 [arXiv: 1708.01256] [INSPIRE].

[21] NNPDF collaboration, Illuminating the photon content of the proton within a global PDF analysis, SciPost Phys. 5 (2018) 008 [arXiv: 1712.07053] [INSPIRE].

[22] L.A. Harland-Lang, A.D. Martin, R. Nathvani and R.S. Thorne, Ad lucem: QED parton distribution functions in the MMHT framework, Eur. Phys. J. C 79 (2019) 811 [arXiv: 1907.02750] [INSPIRE].

[23] V. Bertone, S. Carrazza and J. Rojo, APFEL: A PDF Evolution Library with QED corrections, Comput. Phys. Commun. 185 (2014) 1647 [arXiv:1310.1394] [InSPIRE].

[24] D. de Florian, G.F.R. Sborlini and G. Rodrigo, QED corrections to the Altarelli-Parisi splitting functions, Eur. Phys. J. C 76 (2016) 282 [arXiv:1512.00612] [INSPIRE].

[25] NNPDF collaboration, A first determination of parton distributions with theoretical uncertainties, Eur. Phys. J. C (2019) 79:838 [arXiv:1905.04311] [INSPIRE].

[26] NNPDF collaboration, Parton distributions with theory uncertainties: general formalism and first phenomenological studies, Eur. Phys. J. C 79 (2019) 931 [arXiv:1906.10698] [INSPIRE].

[27] A. Denner and S. Pozzorini, One loop leading logarithms in electroweak radiative corrections. 1. Results, Eur. Phys. J. C 18 (2001) 461 [hep-ph/0010201] [INSPIRE].

[28] A. Denner and S. Pozzorini, One loop leading logarithms in electroweak radiative corrections. 2. Factorization of collinear singularities, Eur. Phys. J. C 21 (2001) 63 [hep-ph/0104127] [INSPIRE].

[29] T. Carli et al., A posteriori inclusion of parton density functions in NLO QCD final-state calculations at hadron colliders: the APPLGRID project, Eur. Phys. J. C 66 (2010) 503 [arXiv:0911.2985] [INSPIRE].

[30] T. Kluge, K. Rabbertz and M. Wobisch, FastNLO: fast $p Q C D$ calculations for PDF fits, in $14^{\text {th }}$ International Workshop on Deep Inelastic Scattering, World Scientific, Singapore (2006), pg. 483 [hep-ph/0609285] [INSPIRE].

[31] FASTNLO collaboration, Theory-data comparisons for jet measurements in hadron-induced processes, arXiv:1109.1310 [INSPIRE]. 
[32] FASTNLO collaboration, New features in version 2 of the fastNLO project, in $20^{\text {th }}$ International Workshop on Deep-Inelastic Scattering and Related Subjects, DESY-PROC-2012-02, (2012), pg. 217 [arXiv: 1208.3641] [INSPIRE].

[33] V. Bertone, R. Frederix, S. Frixione, J. Rojo and M. Sutton, aMCfast: automation of fast NLO computations for PDF fits, JHEP 08 (2014) 166 [arXiv:1406.7693] [INSPIRE].

[34] L. Del Debbio, N.P. Hartland and S. Schumann, MCgrid: projecting cross section calculations on grids, Comput. Phys. Commun. 185 (2014) 2115 [arXiv:1312.4460] [INSPIRE].

[35] E. Bothmann, N. Hartland and S. Schumann, Introducing MCgrid 2.0: projecting cross section calculations on grids, Comput. Phys. Commun. 196 (2015) 617 [INSPIRE].

[36] V. Bertone, S. Carrazza, D. Pagani and M. Zaro, On the impact of lepton PDFs, JHEP 11 (2015) 194 [arXiv: 1508.07002] [INSPIRE].

[37] L. Buonocore, P. Nason, F. Tramontano and G. Zanderighi, Leptons in the proton, JHEP 08 (2020) 019 [arXiv: 2005.06477] [INSPIRE].

[38] S. Frixione, Z. Kunszt and A. Signer, Three jet cross-sections to next-to-leading order, Nucl. Phys. B 467 (1996) 399 [hep-ph/9512328] [INSPIRE].

[39] S. Frixione, A general approach to jet cross-sections in QCD, Nucl. Phys. B 507 (1997) 295 [hep-ph/9706545] [INSPIRE].

[40] S. Catani and M.H. Seymour, The dipole formalism for the calculation of QCD jet cross-sections at next-to-leading order, Phys. Lett. B 378 (1996) 287 [hep-ph/9602277] [INSPIRE].

[41] S. Catani, S. Dittmaier, M.H. Seymour and Z. Trócsányi, The dipole formalism for next-to-leading order QCD calculations with massive partons, Nucl. Phys. B 627 (2002) 189 [hep-ph/0201036] [INSPIRE].

[42] B. Biedermann, A. Denner and M. Pellen, Complete NLO corrections to $W^{+} W^{+}$scattering and its irreducible background at the LHC, JHEP 10 (2017) 124 [arXiv:1708.00268] [INSPIRE].

[43] A. Denner, S. Dittmaier, P. Maierhöfer, M. Pellen and C. Schwan, QCD and electroweak corrections to $W Z$ scattering at the LHC, JHEP 06 (2019) 067 [arXiv:1904.00882] [INSPIRE].

[44] R. Frederix, D. Pagani and M. Zaro, Large NLO corrections in $t \bar{t} W^{ \pm}$and $t \bar{t} t \bar{t}$ hadroproduction from supposedly subleading EW contributions, JHEP 02 (2018) 031 [arXiv: 1711.02116] [INSPIRE].

[45] D. Pagani, H.-S. Shao and M. Zaro, RIP Hbb: how other Higgs production modes conspire to kill a rare signal at the LHC, JHEP 11 (2020) 036 [arXiv:2005.10277] [INSPIRE].

[46] R. Frederix, S. Frixione, F. Maltoni and T. Stelzer, Automation of next-to-leading order computations in QCD: the FKS subtraction, JHEP 10 (2009) 003 [arXiv:0908.4272] [INSPIRE].

[47] R. Frederix, S. Frixione, A.S. Papanastasiou, S. Prestel and P. Torrielli, Off-shell single-top production at NLO matched to parton showers, JHEP 06 (2016) 027 [arXiv: 1603.01178] [INSPIRE]. 
[48] V. Hirschi, R. Frederix, S. Frixione, M.V. Garzelli, F. Maltoni and R. Pittau, Automation of one-loop QCD corrections, JHEP 05 (2011) 044 [arXiv:1103.0621] [INSPIRE].

[49] G. Passarino and M.J.G. Veltman, One loop corrections for $e^{+} e^{-}$annihilation into $\mu^{+} \mu^{-}$ in the Weinberg model, Nucl. Phys. B 160 (1979) 151 [INSPIRE].

[50] A.I. Davydychev, A simple formula for reducing Feynman diagrams to scalar integrals, Phys. Lett. B 263 (1991) 107 [INSPIRE].

[51] A. Denner and S. Dittmaier, Reduction schemes for one-loop tensor integrals, Nucl. Phys. B 734 (2006) 62 [hep-ph/0509141] [INSPIRE].

[52] G. Ossola, C.G. Papadopoulos and R. Pittau, Reducing full one-loop amplitudes to scalar integrals at the integrand level, Nucl. Phys. B 763 (2007) 147 [hep-ph/0609007] [InSPIRE].

[53] F. Cascioli, P. Maierhofer and S. Pozzorini, Scattering amplitudes with open loops, Phys. Rev. Lett. 108 (2012) 111601 [arXiv:1111.5206] [INSPIRE].

[54] P. Mastrolia, E. Mirabella and T. Peraro, Integrand reduction of one-loop scattering amplitudes through Laurent series expansion, JHEP 06 (2012) 095 [Erratum ibid. 11 (2012) 128] [arXiv: 1203.0291] [INSPIRE].

[55] G. Ossola, C.G. Papadopoulos and R. Pittau, CutTools: a program implementing the OPP reduction method to compute one-loop amplitudes, JHEP 03 (2008) 042 [arXiv:0711.3596] [INSPIRE].

[56] T. Peraro, Ninja: automated integrand reduction via Laurent expansion for one-loop amplitudes, Comput. Phys. Commun. 185 (2014) 2771 [arXiv:1403.1229] [INSPIRE].

[57] V. Hirschi and T. Peraro, Tensor integrand reduction via Laurent expansion, JHEP 06 (2016) 060 [arXiv: 1604.01363] [INSPIRE].

[58] A. Denner, S. Dittmaier and L. Hofer, Collier: a fortran-based Complex One-Loop LIbrary in Extended Regularizations, Comput. Phys. Commun. 212 (2017) 220 [arXiv:1604.06792] [INSPIRE].

[59] S. Frixione and B.R. Webber, Matching NLO QCD computations and parton shower simulations, JHEP 06 (2002) 029 [hep-ph/0204244] [INSPIRE].

[60] C. Degrande, C. Duhr, B. Fuks, D. Grellscheid, O. Mattelaer and T. Reiter, UFO - the Universal FeynRules Output, Comput. Phys. Commun. 183 (2012) 1201 [arXiv:1108.2040] [INSPIRE].

[61] A. Denner, S. Dittmaier, M. Roth and D. Wackeroth, Predictions for all processes $e^{+} e^{-} \rightarrow 4$ fermions $+\gamma$, Nucl. Phys. B 560 (1999) 33 [hep-ph/9904472] [INSPIRE].

[62] A. Denner, S. Dittmaier, M. Roth and L.H. Wieders, Electroweak corrections to charged-current $e^{+} e^{-} \rightarrow 4$ fermion processes: technical details and further results, Nucl. Phys. B 724 (2005) 247 [Erratum ibid. 854 (2012) 504] [hep-ph/0505042] [INSPIRE].

[63] R. Frederix, S. Frixione, V. Hirschi, F. Maltoni, R. Pittau and P. Torrielli, Four-lepton production at hadron colliders: aMC@NLO predictions with theoretical uncertainties, JHEP 02 (2012) 099 [arXiv:1110.4738] [INSPIRE].

[64] E.W. Glover and A.G. Morgan, Measuring the photon fragmentation function at LEP, Z. Phys. C 62 (1994) 311 [InSPIRE].

[65] R. Frederix, S. Frixione, V. Hirschi, D. Pagani, H.-S. Shao and M. Zaro, The complete NLO corrections to dijet hadroproduction, JHEP 04 (2017) 076 [arXiv: 1612.06548] [INSPIRE]. 
[66] A. Denner, S. Dittmaier, M. Pellen and C. Schwan, Low-virtuality photon transitions $\gamma^{*} \rightarrow f \bar{f}$ and the photon-to-jet conversion function, Phys. Lett. B 798 (2019) 134951 [arXiv: 1907.02366] [INSPIRE].

[67] S. Frixione, V. Hirschi, D. Pagani, H.S. Shao and M. Zaro, Weak corrections to Higgs hadroproduction in association with a top-quark pair, JHEP 09 (2014) 065 [arXiv: 1407.0823] [INSPIRE].

[68] S. Frixione, V. Hirschi, D. Pagani, H.S. Shao and M. Zaro, Electroweak and QCD corrections to top-pair hadroproduction in association with heavy bosons, JHEP 06 (2015) 184 [arXiv: 1504.03446] [INSPIRE].

[69] D. Pagani, I. Tsinikos and M. Zaro, The impact of the photon PDF and electroweak corrections on $t \bar{t}$ distributions, Eur. Phys. J. C 76 (2016) 479 [arXiv:1606.01915] [INSPIRE].

[70] ATLAS collaboration, Measurement of the high-mass Drell-Yan differential cross-section in pp collisions at $\sqrt{s}=7 \mathrm{TeV}$ with the ATLAS detector, Phys. Lett. B $\mathbf{7 2 5}$ (2013) 223 [arXiv: 1305.4192] [INSPIRE].

[71] CMS collaboration, Measurement of the differential and double-differential Drell-Yan cross sections in proton-proton collisions at $\sqrt{s}=7 \mathrm{TeV}$, JHEP 12 (2013) 030 [arXiv:1310.7291] [INSPIRE].

[72] F. Buccioni, F. Caola, M. Delto, M. Jaquier, K. Melnikov and R. Röntsch, Mixed QCD-electroweak corrections to on-shell Z production at the LHC, Phys. Lett. B $\mathbf{8 1 1}$ (2020) 135969 [arXiv: 2005.10221] [INSPIRE].

[73] U. Baur, O. Brein, W. Hollik, C. Schappacher and D. Wackeroth, Electroweak radiative corrections to neutral current Drell-Yan processes at hadron colliders, Phys. Rev. D 65 (2002) 033007 [hep-ph/0108274] [INSPIRE].

[74] A. Arbuzov et al., One-loop corrections to the Drell-Yan process in SANC. (II). The neutral current case, Eur. Phys. J. C 54 (2008) 451 [arXiv:0711.0625] [InSPIRE].

[75] S. Dittmaier and M. Huber, Radiative corrections to the neutral-current Drell-Yan process in the Standard Model and its minimal supersymmetric extension, JHEP 01 (2010) 060 [arXiv: 0911.2329] [INSPIRE].

[76] S. Alioli et al., Precision studies of observables in $p p \rightarrow W \rightarrow \ell \nu_{\ell}$ and $p p \rightarrow \gamma, Z \rightarrow \ell^{+} \ell^{-}$ processes at the LHC, Eur. Phys. J. C 77 (2017) 280 [arXiv:1606.02330] [inSPIRE].

[77] C. Anastasiou, L.J. Dixon, K. Melnikov and F. Petriello, Dilepton rapidity distribution in the Drell-Yan process at NNLO in QCD, Phys. Rev. Lett. 91 (2003) 182002 [hep-ph/0306192] [INSPIRE].

[78] S. Catani, L. Cieri, G. Ferrera, D. de Florian and M. Grazzini, Vector boson production at hadron colliders: a fully exclusive QCD calculation at NNLO, Phys. Rev. Lett. 103 (2009) 082001 [arXiv: 0903.2120] [INSPIRE].

[79] R. Gavin, Y. Li, F. Petriello and S. Quackenbush, FEWZ 2.0: a code for hadronic Z production at next-to-next-to-leading order, Comput. Phys. Commun. 182 (2011) 2388 [arXiv: 1011.3540] [INSPIRE].

[80] Y. Li and F. Petriello, Combining QCD and electroweak corrections to dilepton production in FEWZ, Phys. Rev. D 86 (2012) 094034 [arXiv:1208.5967] [INSPIRE]. 
[81] R. Boughezal et al., Color singlet production at NNLO in MCFM, Eur. Phys. J. C 77 (2017) 7 [arXiv: 1605.08011] [INSPIRE].

[82] R.D. Ball and A. Deshpande, The proton spin, semi-inclusive processes, and measurements at a future Electron Ion Collider, in From my vast repertoire...: Guido Altarelli's legacy,

A. Levy, S. Forte and G. Ridolfi eds., World Scientific, Singapore (2019), pg. 205 [arXiv: 1801.04842] [INSPIRE].

[83] ATLAS collaboration, Measurements of top-quark pair differential cross-sections in the lepton+jets channel in pp collisions at $\sqrt{s}=8 \mathrm{TeV}$ using the ATLAS detector, Eur. Phys. J. $C 76$ (2016) 538 [arXiv:1511.04716] [INSPIRE].

[84] CMS collaboration, Measurement of the differential cross section for top quark pair production in pp collisions at $\sqrt{s}=8$ TeV, Eur. Phys. J. C 75 (2015) 542 [arXiv: 1505.04480] [INSPIRE].

[85] M. Czakon, N.P. Hartland, A. Mitov, E.R. Nocera and J. Rojo, Pinning down the large-x gluon with NNLO top-quark pair differential distributions, JHEP 04 (2017) 044 [arXiv: 1611.08609] [INSPIRE].

[86] S. Bailey and L. Harland-Lang, Differential top quark pair production at the LHC: challenges for PDF fits, Eur. Phys. J. C 80 (2020) 60 [arXiv:1909.10541] [INSPIRE].

[87] M. Kadir, A. Ablet, S. Dulat, T.-J. Hou and I. Sitiwaldi, The impact of ATLAS and CMS single differential top-quark pair measurements at $\sqrt{s}=8 \mathrm{TeV}$ to CTEQ-TEA PDFs, arXiv:2003.13740 [INSPIRE].

[88] M. Czakon, D. Heymes, A. Mitov, D. Pagani, I. Tsinikos and M. Zaro, Top-pair production at the LHC through NNLO QCD and NLO EW, JHEP 10 (2017) 186 [arXiv:1705.04105] [INSPIRE].

[89] J.H. Kühn, A. Scharf and P. Uwer, Electroweak effects in top-quark pair production at hadron colliders, Eur. Phys. J. C 51 (2007) 37 [hep-ph/0610335] [INSPIRE].

[90] W. Bernreuther and Z.-G. Si, Distributions and correlations for top quark pair production and decay at the Tevatron and LHC, Nucl. Phys. B 837 (2010) 90 [arXiv:1003.3926] [INSPIRE].

[91] W. Hollik and D. Pagani, The electroweak contribution to the top quark forward-backward asymmetry at the Tevatron, Phys. Rev. D 84 (2011) 093003 [arXiv:1107.2606] [InSPIRE].

[92] J.H. Kühn and G. Rodrigo, Charge asymmetries of top quarks at hadron colliders revisited, JHEP 01 (2012) 063 [arXiv:1109.6830] [INSPIRE].

[93] W. Bernreuther and Z.-G. Si, Top quark and leptonic charge asymmetries for the Tevatron and LHC, Phys. Rev. D 86 (2012) 034026 [arXiv:1205.6580] [INSPIRE].

[94] A. Denner and M. Pellen, NLO electroweak corrections to off-shell top-antitop production with leptonic decays at the LHC, JHEP 08 (2016) 155 [arXiv: 1607.05571] [INSPIRE].

[95] M. Czakon, D. Heymes, A. Mitov, D. Pagani, I. Tsinikos and M. Zaro, Top-quark charge asymmetry at the LHC and Tevatron through NNLO QCD and NLO EW, Phys. Rev. D 98 (2018) 014003 [arXiv:1711.03945] [INSPIRE].

[96] M. Czakon, D. Heymes, A. Mitov, D. Pagani, I. Tsinikos and M. Zaro, Top-quark pair production at NNLO QCD + NLO EW accuracy: Tevatron results, in $10^{\text {th }}$ International Workshop on Top Quark Physics, (2017) [arXiv:1712.04842] [INSPIRE]. 
[97] C. Gütschow, J.M. Lindert and M. Schönherr, Multi-jet merged top-pair production including electroweak corrections, Eur. Phys. J. C 78 (2018) 317 [arXiv:1803.00950] [INSPIRE].

[98] M.L. Czakon et al., NNLO versus NLO multi-jet merging for top-pair production including electroweak corrections, in $11^{\text {th }}$ International Workshop on Top Quark Physics, (2019) [arXiv: 1901.04442] [INSPIRE].

[99] M.L. Czakon et al., Top quark pair production at complete NLO accuracy with NNLO+NNLL' corrections in QCD, Chin. Phys. C 44 (2020) 083104 [arXiv:1901.08281] [INSPIRE].

[100] M. Czakon, D. Heymes and A. Mitov, Dynamical scales for multi-TeV top-pair production at the LHC, JHEP 04 (2017) 071 [arXiv:1606. 03350] [INSPIRE].

[101] ATLAS collaboration, Measurement of the transverse momentum and $\phi_{\eta}^{*}$ distributions of Drell-Yan lepton pairs in proton-proton collisions at $\sqrt{s}=8 \mathrm{TeV}$ with the ATLAS detector, Eur. Phys. J. C 76 (2016) 291 [arXiv:1512.02192] [InSPIRE].

[102] CMS collaboration, Measurement of the $Z$ boson differential cross section in transverse momentum and rapidity in proton-proton collisions at 8 TeV, Phys. Lett. B 749 (2015) 187 [arXiv:1504.03511] [INSPIRE].

[103] M. Czakon, P. Fiedler and A. Mitov, Total top-quark pair-production cross section at hadron colliders through $O\left(\alpha_{S}^{4}\right)$, Phys. Rev. Lett. 110 (2013) 252004 [arXiv:1303.6254] [INSPIRE].

[104] M. Czakon, P. Fiedler and A. Mitov, Resolving the Tevatron top quark forward-backward asymmetry puzzle: fully differential next-to-next-to-leading-order calculation, Phys. Rev. Lett. 115 (2015) 052001 [arXiv: 1411.3007] [INSPIRE].

[105] M. Czakon, D. Heymes and A. Mitov, High-precision differential predictions for top-quark pairs at the LHC, Phys. Rev. Lett. 116 (2016) 082003 [arXiv:1511.00549] [INSPIRE].

[106] M. Czakon, P. Fiedler, D. Heymes and A. Mitov, NNLO QCD predictions for fully-differential top-quark pair production at the Tevatron, JHEP 05 (2016) 034 [arXiv: 1601.05375] [INSPIRE].

[107] S. Catani, S. Devoto, M. Grazzini, S. Kallweit, J. Mazzitelli and H. Sargsyan, Top-quark pair hadroproduction at next-to-next-to-leading order in QCD, Phys. Rev. D 99 (2019) 051501 [arXiv: 1901.04005] [INSPIRE].

[108] A. Behring, M. Czakon, A. Mitov, A.S. Papanastasiou and R. Poncelet, Higher order corrections to spin correlations in top quark pair production at the LHC, Phys. Rev. Lett. 123 (2019) 082001 [arXiv:1901.05407] [INSPIRE].

[109] S. Catani, S. Devoto, M. Grazzini, S. Kallweit and J. Mazzitelli, Top-quark pair production at the LHC: fully differential QCD predictions at NNLO, JHEP 07 (2019) 100 [arXiv: 1906. 06535] [INSPIRE].

[110] S. Catani, S. Devoto, M. Grazzini, S. Kallweit and J. Mazzitelli, Top-quark pair hadroproduction at NNLO: differential predictions with the $\overline{M S}$ mass, JHEP 08 (2020) 027 [arXiv: 2005. 00557] [INSPIRE].

[111] M. Czakon, A. Mitov and R. Poncelet, NNLO QCD corrections to leptonic observables in top-quark pair production and decay, arXiv:2008.11133 [INSPIRE]. 
[112] CMS collaboration, Measurements of differential $Z$ boson production cross sections in proton-proton collisions at $\sqrt{s}=13 \mathrm{TeV}$, JHEP 12 (2019) 061 [arXiv:1909.04133] [INSPIRE].

[113] R. Boughezal et al., Z-boson production in association with a jet at next-to-next-to-leading order in perturbative QCD, Phys. Rev. Lett. 116 (2016) 152001 [arXiv:1512.01291] [INSPIRE].

[114] R. Boughezal, X. Liu and F. Petriello, Phenomenology of the Z-boson plus jet process at NNLO, Phys. Rev. D 94 (2016) 074015 [arXiv:1602.08140] [InSPIRE].

[115] R. Boughezal, X. Liu and F. Petriello, A comparison of NNLO QCD predictions with $7 \mathrm{TeV}$ ATLAS and CMS data for $V+$ jet processes, Phys. Lett. B 760 (2016) 6 [arXiv: 1602.05612] [INSPIRE].

[116] A. Gehrmann-De Ridder, T. Gehrmann, E.W.N. Glover, A. Huss and T.A. Morgan, Precise $Q C D$ predictions for the production of a $Z$ boson in association with a hadronic jet, Phys. Rev. Lett. 117 (2016) 022001 [arXiv:1507.02850] [INSPIRE].

[117] A. Gehrmann-De Ridder, T. Gehrmann, E.W.N. Glover, A. Huss and T.A. Morgan, The NNLO QCD corrections to $Z$ boson production at large transverse momentum, JHEP 07 (2016) 133 [arXiv: 1605.04295] [INSPIRE].

[118] A. Gehrmann-De Ridder, T. Gehrmann, E.W.N. Glover, A. Huss and D.M. Walker, Next-to-next-to-leading-order QCD corrections to the transverse momentum distribution of weak gauge bosons, Phys. Rev. Lett. 120 (2018) 122001 [arXiv:1712.07543] [INSPIRE].

[119] W. Bizon et al., The transverse momentum spectrum of weak gauge bosons at $N^{3} L L+N N L O$, Eur. Phys. J. C 79 (2019) 868 [arXiv: 1905.05171] [INSPIRE].

[120] R. Boughezal, A. Guffanti, F. Petriello and M. Ubiali, The impact of the LHC Z-boson transverse momentum data on PDF determinations, JHEP 07 (2017) 130 [arXiv: 1705.00343] [INSPIRE].

[121] J.H. Kühn, A. Kulesza, S. Pozzorini and M. Schulze, One-loop weak corrections to hadronic production of $Z$ bosons at large transverse momenta, Nucl. Phys. B 727 (2005) 368 [hep-ph/0507178] [INSPIRE].

[122] A. Denner, S. Dittmaier, T. Kasprzik and A. Muck, Electroweak corrections to dilepton+jet production at hadron colliders, JHEP 06 (2011) 069 [arXiv:1103.0914] [INSPIRE].

[123] W. Hollik, B.A. Kniehl, E.S. Scherbakova and O.L. Veretin, Electroweak corrections to Z-boson hadroproduction at finite transverse momentum, Nucl. Phys. B 900 (2015) 576 [arXiv: 1504.07574] [INSPIRE].

[124] S. Kallweit, J.M. Lindert, P. Maierhofer, S. Pozzorini and M. Schönherr, $N L O Q C D+E W$ predictions for $V+$ jets including off-shell vector-boson decays and multijet merging, JHEP 04 (2016) 021 [arXiv: 1511.08692] [INSPIRE].

[125] ATLAS collaboration, Measurement of the Drell-Yan triple-differential cross section in pp collisions at $\sqrt{s}=8 \mathrm{TeV}$, JHEP 12 (2017) 059 [arXiv:1710.05167] [INSPIRE].

[126] ATLAS collaboration, Measurement of the double-differential high-mass Drell-Yan cross section in pp collisions at $\sqrt{s}=8 \mathrm{TeV}$ with the ATLAS detector, JHEP 08 (2016) 009 [arXiv: 1606.01736] [INSPIRE].

[127] D. Bardin et al., SANC integrator in the progress: QCD and EW contributions, JETP Lett. 96 (2012) 285 [arXiv: 1207.4400] [INSPIRE]. 
[128] S.G. Bondarenko and A.A. Sapronov, NLO EW and QCD proton-proton cross section calculations with mcsanc-v1.01, Comput. Phys. Commun. 184 (2013) 2343 [arXiv:1301.3687] [INSPIRE].

[129] E. Barberio, B. van Eijk and Z. Was, PHOTOS: a universal Monte Carlo for QED radiative corrections in decays, Comput. Phys. Commun. 66 (1991) 115 [INSPIRE].

[130] E. Barberio and Z. Was, PHOTOS: a universal Monte Carlo for QED radiative corrections. Version 2.0, Comput. Phys. Commun. 79 (1994) 291 [INSPIRE].

[131] P. Golonka and Z. Was, PHOTOS Monte Carlo: a precision tool for QED corrections in Z and $W$ decays, Eur. Phys. J. C 45 (2006) 97 [hep-ph/0506026] [INSPIRE].

[132] C.M. Carloni Calame et al., Precision measurement of the $W$-boson mass: theoretical contributions and uncertainties, Phys. Rev. D 96 (2017) 093005 [arXiv:1612.02841] [INSPIRE].

[133] A. Buckley et al., LHAPDF6: parton density access in the LHC precision era, Eur. Phys. J. C 75 (2015) 132 [arXiv:1412.7420] [INSPIRE]. 